Meta

Journal des traducteurs

Translators' Journal

\title{
Index du volume 28
}

Volume 28, numéro 4, décembre 1983

URI : https://id.erudit.org/iderudit/003181ar

DOI : https://doi.org/10.7202/003181ar

Aller au sommaire du numéro

Éditeur(s)

Les Presses de l'Université de Montréal

ISSN

0026-0452 (imprimé)

1492-1421 (numérique)

Découvrir la revue

Citer ce document

(1983). Index du volume 28. Meta, 28(4), 433-466.

https://doi.org/10.7202/003181ar

Ce document est protégé par la loi sur le droit d'auteur. L'utilisation des services d'Érudit (y compris la reproduction) est assujettie à sa politique d'utilisation que vous pouvez consulter en ligne.

https://apropos.erudit.org/fr/usagers/politique-dutilisation/ 


\section{INDEX DU VOLUME 28}

\section{A. ARTICLES}

Aphek, Edna : The Means is the Message : On the Intranslability of a Hebrew Text, vol. 28, $\mathrm{n}^{\circ} 1$, p. 57. Aziz, Yowelle Y. : Transliteration of English Proper Nouns into Arabic, vol. 28, $\mathrm{n}^{\circ}$ 1, p. 70.

Bourjea, Michelle : Le traducteur ou par-delà l'esclave-maître, vol. 28, $n^{\circ} 1$, p. 35.

Clas, André : Éditorial, vol. 28, $\mathrm{n}^{\circ} 2$, p. 115.

Edebiri, Uionmwan : Literary Translation in Nigeria, vol. 28, $\mathrm{n}^{\circ} 1$, p. 27.

Gémar, J.-C. : De la pratique à la théorie,l'apport des praticiens à la théorie générale de la traduction, vol. 28 , $n^{\circ} 4$, p. 323.

Gile, Daniel : Aspects méthodologiques de l'évaluation de la qualité du travail en interprétation simultanée, vol. $28, n^{\circ} 3$, p. 236.

Gile, Daniel : La traduction et l'interprétation en Océanie, vol. 28, nº 1, p. 17.

Haerens, N. : Souvenirs d'un être universel. L'interprète interplanétaire, vol. 28, no 3, p. 227.

Harris, Brian : Translation, Translation Teaching, and the Transfer of Technology, vol. 28, $\mathrm{n}^{\circ} 1, \mathrm{p} .5$.

Klett, Estella : Virtualité et textualité en traduction, vol. 28 , no 1 , p. 48.

Moskowitz, Daniel : La traduction technique en France, vol. 28, no 1, p. 90

Mossop, Brian : The Translator as Rapporteur : A Concept for Training and Self-improvement, vol. 28, $\mathrm{n}^{\circ} 3$, p. 224.

Nicklen, Louise : Interpretation, Translation and Communication in Canada's Far North, vol. 28, n², p. 153.

Nishiyama, Sen : Translation and Interpretation in Japan, vol. $28, n^{\circ} 1$, p. 95.

Osers, Ewald : The Current Situation in the United Kingdom, vol. 28, $\mathrm{n}^{\circ} 1$, p. 85.

Patterson, John F. : Antonine Maillet, traduite ou trahie ?, vol. 28, $\mathrm{n}^{\circ} 4$, p. 352.

Priso, Thérèse : Les traducteurs camerounais face aux problèmes linguistiques, vol. 28, no 1, p. 20.

Schneider, Thomas : Some notes on machine aids for translators, vol. 28, n॰ 4, p. 344.

Thien, Ton That : Linguistique et traduction : propos de traducteur, vol. $28, \mathrm{n}^{\circ} 2, \mathrm{p} .130$

Tobin, Yishai : The Means is the Message : On the Intranslability of a Hebrew Text, vol. $28, n^{\circ} 1$, p. 57 Toury, Gideon : Sharing Relevant Features. An Exercice in Optimal Translating, vol. 28, no 2, p. 116.

Van Hoof, Henri : Dans les coulisses de la traduction, vol. 28, no 4 , p. 334.

Vardar, Bérke : La troisième rencontre mondiale de l'AUPELF et les problèmes de traduction, vol. $28, \mathrm{n}^{\circ} 1$, p. 93 .

\section{B. ÉTUDES TERMINOLOGIQUES ET LINGUISTIQUES}

Bagge, C. : Analyse sémantique comparative des structures des vocabulaires scientifiques anglais et français, vol. $28, n^{\circ} 4$, p. 391 .

Bélanger, Gilles : La nomenclature des composés organiques, vol. 28, n $^{\circ} 2$, p. 166

Boult, Raynald : Proceeding(s) : procédure(s) ou non ?, vol. 28, no 2, p. 172.

Di-Lillo, Antoine : Il n'y a pas de suffixe -ateur en français, voyons ! (III), vol. 28, no 2, p. 157.

Goyette, Lucie : Le chauffage solaire, vol. $28, \mathrm{n}^{\circ} 3$, p. 283.

Jammal, Amal : L'hypertension artérielle et les singularités de son vocabulaire, vol. $28, n^{\circ} 4$, p. 376.

Jarema, Gonia : $\dot{A}$ propos de lexicographie terminologique, vol. $28, n^{\circ} 3$, p. 279.

Journal officiel (1983) français : Enrichissement du vocabulaire de l'audio-visuel et de la publicité, vol. 28, no 4 , p. 399. 
Lethuillier, Jacques : À propos de lexicographie terminologique, vol. 28, no 3, p. 279.

Lethuillier, Jacques : Le langage de la régulation, vol. 28, $\mathrm{n}^{\circ} 4$, p. 365.

Méchin, Bernard : Proceeding(s) : procédure(s) ou non ?, vol. 28, nº 2, p. 172.

Proulx, Marie : Étude terminologique des scies manuelles (I), vol. 28, $\mathrm{n}^{\circ} 2$, p. 192.

Proulx, Marie : Étude terminologique des scies manuelles (II), vol. 28, $\mathrm{n}^{\circ} 4$, p. 358.

Renaud, Alix : Qu'est-ce que la stéréophonie ?, vol. 28, $\mathrm{n}^{\circ} 2$, p. 186.

Serré, Robert : Vocabulaire contextuel du bâtiment, vol. 28, no 3, p. 292.

Taillat, D. : Mini-lexique de droit public, vol. $28, \mathrm{n}^{\circ} 4$, p. 369.

Weil-Brenner, Richard : Boisson alcoolique ou boisson alcoolisée ?, vol. 28, nº 2, p. 178.

\section{DOCUMENTATION}

Serré, Robert : Ouvrages récents qui englobent un lexique ou un glossaire, vol. $28, n^{\circ} 2$, p. 203 ; vol. 28, n⿳ 3 , p. 297 ; vol. 28, n $^{\circ} 4$, p. 409.

Spilka, Irène V. : Bibliographie, vol. $28, n^{\circ} 2$, p. 202 ; vol. $28, n^{\circ} 3$, p. 296 ; vol. 28, no 4, p. 408.

\section{COMPTES RENDUS}

Bélanger, Gilles : Dictionnaire de l'industrie du gaz, vol. 28, n³, p. 297.

Binda, Maurizia : Le business de la traduction, vol. 28, no 3, p. 301.

Boisvert, Anne : La Créativité lexicale, vol. 28, no 3, p. 299.

Darbelnet, Jean : Dictionnaire de la linguistique, vol. 28, no $^{\circ}$, p. 410.

Gémar, Jean-Claude : Dictionnaire de la comptabilité, vol. $28, n^{\circ} 4$, p. 414

Gémar, Jean-Claude : L'interprétation auprès des tribunaux, vol. $28, \mathrm{n}^{\circ} 2$, p. 213.

Goffin, Roger : Inventaire des particularités lexicales du français en Afrique noire, vol. $28, n^{\circ} 3$, p. 302.

Henry, Ronald : Simultaneous Interpreting, a practise book, vol. 28, no 4 , p. 410.

Jarema, Gonia : Textverstehen und übersetzen. Ouvertures sur la traduction, vol. 28, $\mathbf{n}^{\circ}$ 2, p. 206.

Kittredge, Richard : Practical Experience of Machine Translation, vol. 28, no 2, p. 209.

Lethuillier, Jacques : Dictionnaire du génie automobile, vol. $28, n^{\circ} 4$, p. 412.

Lethuillier, Jacques : Le Papier - Procédés et matériel, vol. 28, no 3, p. 304

Mel'cuk, Igor : Computer in der iubersetzungswissenschaft, vol. $28, n^{\circ} 2$, p. 210.

Meyer, Ingrid : Semiotik und Übersetzen, vol. $28, n^{\circ} 3$, p. 300.

Parmentier, Michel A. : La Traduction de l'anglais, Théorie et pratique, vol. 28, no 4, p. 412.

Spilka, Irène V. : Collection of Alliterates and Rhyming Doublets used by the English, vol. 28, no 2, p. 204.

Spilka, Irène V. : La traduction simultanée, fondements théoriques, vol. $28, \mathrm{n}^{\mathrm{o}} 2, \mathrm{p}$. 205.

Spilka, Irène V. : Teoria y practica de la traduccion, primer encuentro internacional de traductores, vol. 28 , $\mathrm{n}^{\circ} 2$, p. 205.

\section{E. BLOC NOTES}

Bossé-Andrieu, J. : Colloque : Traduction et qualité de langue, vol. 28, $\mathbf{n}^{\circ} 2$, p. 220.

Darbelnet, Jean : La stylistique comparée du français et de l'anglais, vol. $28, \mathrm{n}^{\circ} 4$, p. 416.

Henry, Ronald : T.I.E.S. Symposium '83, vol. 28, nº 3, p. 315.

Infoterm : Infoterm Newsletter 27, vol. 28, $n^{\circ} 2$, p. 221.

Larose, Robert : Colloque de traduction en Angleterre, vol. 28, no 4, p. 429.

Richer, Suzanne : Gestion et organisation de la documentation au bureau des traductions du Canada, vol. 28 , $n^{\circ} 2$, p. 217.

Rodrigue, Nelson: La Banque de terminologie du Gouvernement canadien et l'ATIO, vol. 28, n 2, p. 215.

Roth, Armand : L'enseignement des langues dans le secondaire et la traduction, vol. $28, \mathrm{n}^{\circ} 3, \mathrm{p} .310$.

Séguinot, Candace : A Notional Approach to the Teaching of English Grammar, vol. 28, no 3, p. 306.

Vinay, Jean-Paul : SCFA Revisited, vol. 28, n $^{\circ} 4$, p. 416.

Vouvé, Solange : Le congrès de la SILF et la traductio, vol. 28, $n^{\circ} 4$, p. 428.

Yesufu, Abdul R. : A Note on David Diop's " un Berger" in le Temps du martyre, vol. 28, no 3, p. 308. 


\section{Index français des mots et des sujets traités}

Abaisser les chiffres tensionnels, vol. 28, $n^{\circ} 4$, p. 386.

Abandonner le traitement, vol. $28, n^{\circ} 4$, p. 387.

Abîmer, vol. $28, n^{\circ} 3$, p. 304

About, vol. 28, no 4, p. 361 .

Abrègement, vol. 28, no 3, p. 304

Abréviation, vol. 28, no 3, p. 299.

Absence d'article, vol. $28, n^{\circ} 1$, p. 39

Absorbeur, vol. 28, no 3, p. 288.

Absorbeur-échangeur, vol. $28, n^{\circ} 3$, p. 288

Absorption, vol. 28, no 2, p. 161.

Absorptivité, vol. $28, \mathrm{n}^{\circ} 3$, p. 288

Accélérateur, vol. $28, \mathrm{n}^{\circ} 3$, p. 289

Accélération, vol. $28, \mathrm{n}^{\circ} 2$, p. 161

Accommoder, vol. $28, \mathrm{n}^{\circ} 3$, p. 303.

Accompagnateur, vol. 28, no 2, p. 160.

Accroche, vol. 28, no 4, p. 399.

ACCT, vol. $28, \mathrm{n}^{\circ} 1$, p. 93

Accumulateur à pierres, vol. $28, n^{\circ} 3$, p. 289.

Accumulation thermique solaire, vol. $28, \mathrm{n}^{\circ} 3$, p. 289.

Achat groupé, vol. $28, \mathrm{n}^{\circ} 4$, p. 399.

Acide chloro-13 dioxa-6,12 thia-3, vol. 28, $\mathrm{n}^{\circ} 2$, p. 170.

Acide cyclohexane carbodithioïque, vol. $28, n^{\circ} 2$, p. 169.

Acide naphtalène-acétique, vol. $28, \mathrm{n}^{\circ} 2$, p. 169.

Acquiescement, vol. $28, n^{\circ} 4$, p. 387.

Acte, vol. $28, \mathrm{n}^{\circ} 2$, p. 162 ; vol. $28, \mathrm{n}^{\circ} 2$, p. 175 ; vol. $28, n^{\circ} 4$, p. 373 .

Acte de dissection, vol. 28, no 4, p. 323.

Acte de poursuite, vol. $28, \mathrm{n}^{\circ} 2$, p. 174.

Acte (de procédure), vol. $28, n^{\circ} 2$, p. 175

Acte de reconnaissance, vol. $28, n^{\circ} 4$, p. 370

Acte d'instruction, vol. $28, \mathrm{n}^{\circ} 2$, p. 174 ; vol. 28 , $\mathrm{n}^{\circ} 2$, p. 175.

Acte valable, vol. $28, \mathrm{n}^{\circ} 2, \mathrm{p} .175$.

Action, vol. $28, n^{\circ} 2$, p. 148 ; vol. $28, n^{\circ} 2$, p. 162.

Action en justice, vol. $28, \mathrm{n}^{\circ} \mathrm{2}$, p. 173.

Action translinguistique, vol. $28, \mathrm{n}^{\circ} 1$, p. 44.

Actionneur, vol. $28, n^{\circ} 4$, p. 367

Activité, vol. $28, n^{\circ} 2$, p. 173.

Activité traductive, vol. $28, n^{\circ} 2$, p. 131.

Activité traductrice, vol. 28, no 1, p. 46 .

Activité traduisante, vol. $28, n^{\circ} 1$, p. 18 ; vol. 28 , $n^{\circ} 2$, p. 207.

Activités langagières, centre d', vol. $28, \mathrm{n}^{\circ} 1$, p. 94 Actualisation, vol. 28, no 1, p. 52.

Adaptation, vol. 28, $\mathrm{n}^{\circ} 3$, p. 255.

Addition, réaction d', vol. $28, \mathrm{n}^{\circ} 2$, p. 168.
Adhérer, vol. $28, n^{\circ} 2$, p. 160.

Adhésif, vol. $28, \mathrm{n}^{\circ} 2$, p. 160

Adhésion, vol. $28, \mathrm{n}^{\circ} 2$, p. 160

Adifossi, vol. 28, no 3, p. 303.

Adjectif, vol. $28, n^{\circ} 2$, p. 160

Adjectif démonstratif, vol. $28, n^{\circ} 1$, p. 39

Adjectif possessif, vol. $28, n^{\circ} 1$, p. 39.

Adjoint d'arrondissement, vol. $28, \mathrm{n}^{\circ} 1$, p. 23.

Administrateur, vol. 28, no 2, p. 160.

Administration, vol. $28, n^{\circ} 2$, p. 160

Administration locale, vol. $28, \mathrm{n}^{\circ} 1$, p. 23.

Adsorption, vol. 28, $\mathrm{n}^{\circ} 2$, p. 161.

Aérotherme, vol. 28, no 3, p. 289.

Affaire, vol. $28, \mathrm{n}^{\circ} 2$, p. 174.

Affaire courante, vol. $28, n^{\circ} 4$, p. 375 .

Affixe numérique littéral, vol. $28, \mathrm{n}^{\circ} 2$, p. 170.

Afrique noire, vol. 28, no $^{\circ} 3$, p. 302.

A gent, vol. $28, n^{\circ} 2$, p. 148.

Agent bêta adrénolytique, vol. 28 , no 4 , p. 388 .

Agent du développement communautaire, vol. 28 , no 1, p. 23.

Agglutination, vol. $28, \mathrm{n}^{\circ} 2$, p. 161.

Agrafe, vol. 28, no 3, p. 292.

Agresseur, vol. $28, \mathrm{n}^{\circ} 2$, p. 160.

Agression, vol. $28, \mathrm{n}^{\circ} 2$, p. 160 .

Aguichage, vol. $28, n^{\circ} 4$, p. 400 .

Aguiche, vol. 28, no 4, p. 400 .

AIIC, vol. $28, n^{\circ} 3$, p. 236.

Air théorique, vol. $28, \mathrm{n}^{\circ} 3$, p. 298.

Ajourner, vol. $28, n^{\circ} 4$, p. 370

Ajoute, vol. 28, no 3, p. 303.

Alcane, vol. $28, \mathrm{n}^{\circ} 2$, p. 170

Alcool, vol. $28, \mathrm{n}^{\circ} 2$, p. 168.

Alcool éthylique, vol. $28, \mathrm{n}^{\circ} 2$, p. 178.

Alcool méthylique, vol. $28, \mathrm{n}^{\circ} 2$, p. 168 .

Alcoolique, vol. $28, \mathrm{n}^{\circ} 2$, p. 180.

Alcoolisation, vol. $28, \mathrm{n}^{\circ} 2$, p. 183

Alcooliser, vol. $28, \mathrm{n}^{\circ} 2$, p. 181 ; vol. $28, \mathrm{n}^{\circ} 2$, p. 183.

Alcooliser, s', vol. 28, n $^{\circ} 2$, p. 184

Alcoolisme, vocabulaire de l', vol. $28, n^{\circ} 2$, p. 180

Alcoomanie, vol. $28, n^{\circ} 2$, p. 180.

Allège, vol. 28, no $^{\circ}$, p. 294.

Allomorphie, règle d', vol. $28, \mathrm{n}^{\circ} 2, \mathrm{p} .163$.

Alternatif, vol. $28, n^{\circ} 2$, p. 160 .

Amar, vol. $28, n^{\circ} 1$, p. 37

Amarillo, vol. 28, no $^{\circ}$, p. 51

Amateur, vol. $28, \mathrm{n}^{\circ} 2$, p. 160 .

Ambar, vol. $28, \mathrm{n}^{\circ} 1$, p. 51.

Ambi, vol. 28 , no 3, p. 304

Amer, vol. 28, no 2, p. 185.

Amiante, terminologie de $1^{\prime}$, vol. 28 , no 3, p. 282

Ampli, vol. 28 , no 2 , p. 191 .

Ampli-syntoniseur, vol. 28, n० 4, p. 400

Amplificateur, vol. $28, n^{\circ} 2$, p. 191.

Amplificateur de sonorisation, vol. $28, n^{\circ} 4$, p. 400

Amplitude, modulation d', vol. $28, n^{\circ} 2$, p. 192

Analyse componentielle, vol. $28, n^{\circ} 2$, p. 140.

Analyse componentionnelle, vol. $28, \mathrm{n}^{\circ} 2$, p. 148

Analyse en constituants immédiats, vol. $28, \mathrm{n}^{\circ} 2$, p. 151.

Analyse logique, vol. $28, n^{\circ} 2$, p. 148 .

Analyse sémantique comparative, vol. $28, n^{\circ} 4$ p. 391.

Angiotensinogène, vol. $28, n^{\circ} 4$, p. 379.

Angle d'attaque, vol. 28, $\mathrm{n}^{\circ} 2, \mathrm{p} .195$. 
Anhydride acétique, vol. $28, \mathrm{n}^{\circ} 2$, p. 169 .

Anhydro-, vol. $28, \mathrm{n}^{\circ} 2$, p. 168

Anis blanc, vol. 28, no 2, p. 184.

Anis coloré, vol. $28, \mathrm{n}^{\circ} 2$, p. 185

Anisette, vol. $28, \mathrm{n}^{\circ} 2$, p. 185 .

Anneau de fer, vol. 28, no 2 , p. 194.

Annulation, vol. $28, n^{\circ} 4$, p. 375 .

Annuler, vol. $28, n^{\circ} 4$, p. 375 .

Anthropologie linguistique, vol. 28 , no 1, p. 48 .

Antihypertenseur, vol. 28 , no 4 , p. 378 .

Antonine Maillet, traduite ou trahie ?, vol. 28, $n^{\circ} 4$, p. 352 .

Aorte ascendante, vol. $28, n^{\circ} 4$, p. 383 .

Aortique droite, vol. 28, no 4, p. 384 .

Apéritif à base de vin et vermouth, vol. $28, n^{\circ} 2$, p. 184.

Appareil, vol. 28, no 3, p. 304.

Appareil cardio-vasculaire, vol. 28 , no 4 , p. 379

Appareil circulatoire, vol. $28, \mathrm{n}^{\circ} 4$, p. 379.

Appareil juxtaglomérulaire, vol. 28, no 4 , p. 380 .

Appel d'offres, vol. $28, n^{\circ} 4$, p. 375 .

Appellation d'origine contrôlée, vol. $28, \mathrm{n}^{\circ} 2$, p. 185 .

Apport des praticiens, vol. $28, n^{\circ} 4$, p. 323.

Apport solaire, vol. 28, no 3, p. 289.

Arasement, vol. 28, n०2, p. 196.

Aratoire, vol. 28, no 2, p. 160.

Arbre, vol. $28, n^{\circ} 3$, p. 280.

Arbre anglais, vol. $28, n^{\circ} 4$, p. 393.

Arbre français, vol. $28, n^{\circ} 4$, p. 393 .

Arc de l'aorte, vol. 28 , no 4, p. 383.

Architecture solaire, vol. 28, no 3, p. 289.

Ardo, vol. $28, \mathrm{n}^{\circ} 1$, p. 25.

Ardorate, vol. $28, n^{\circ} 1$, p. 25

Argument, vol. $28, n^{\circ} 2$, p. 148.

Armagnac, vol. 28, no 2, p. 185.

Armateur, vol. $28, \mathrm{n}^{\circ} 2$, p. 160

Arrangeur, vol. 28, no $^{\circ}$, p. 44.

Artère péronière, vol. $28, n^{\circ} 4$, p. 384 .

Artère poplitée, vol. $28, \mathrm{n}^{\circ} 4$, p. 384 .

Artère pulmonaire, vol. $28, \mathrm{n}^{\circ} 4$, p. 383 .

Artère tibiale postérieure, vol. $28, n^{\circ} 4$, p. 384 .

Article, vol. 28, no 4, p. 371.

Article, absence d', vol. 28, no $^{\circ} 1$, p. 39.

Article défini, vol. 28 , n० 1 , p. 39 .

Article indéfini, vol. $28, n^{\circ} 1$, p. 39.

Articulation du domaine, vol. 28 , no 3, p. 280.

Asepsie, vol. $28, \mathrm{n}^{\circ} 2$, p. 162.

Aseptique, vol. $28, n^{\circ} 2$, p. 162.

Aspect traductionnel, vol. $28, n^{\circ} 4$, p. 330

Assemblage bras-traverse, vol. $28, \mathrm{n}^{\circ} 2$, p. 196.

Assemblage d'atomes, vol. $28, n^{\circ} 2$, p. 167.

Assemblage tenon-mortaise, vol. 28, no 2, p. 193.

Assemblée des chefs traditionnels, vol. $28, \mathrm{n}^{\circ} 1$, p. 23.

Assemblée nationale, vol. 28, no 1, p. 22.

Assesseur, vol. 28, $\mathrm{n}^{\circ} 2$, p. 160.

Assez bien, vol. 28, no $^{\circ}$, p. 303

Assise, vol. 28, no 3, p. 293.

-ateur, suffixe, vol. 28, n $^{\circ} 2$, p. 157.

-ation, vol. $28, n^{\circ} 2$, p. 158.

Atome, élimination d', vol. 28 , no 2 , p. 168.

Atomes, assemblage d', vol. 28 , no 2, p. 167.

Atrio-ventriculaire, vol. 28 , no 4 , p. 381 .

Attache, vol. $28, n^{\circ} 3$, p. 292

Attaque, angle d', vol. 28, n $^{\circ} 2$, p. 195.
Attentif, vol. $28, \mathrm{n}^{\circ} 2$, p. 162.

Attention, vol. 28, no 2, p. 162.

Audience, vol. 28, no 2, p. 176.

Audio-visuel, influence de l', vol. 28 , no 3, p. 312.

Audiophile, vol. 28, no 2, p. 191.

Audiophonie, vol. 28, no 2, p. 191

Audiophonie, lexique de l', vol. 28, n० 2, p. 191.

Auditeur, vol. 28 , no 2 , p. 160 ; vol. 28 , no 3 , p. 237.

Audition, vol. $28, \mathrm{n}^{\circ} 2$, p. 160

Audition binaurale, vol. $28, n^{\circ} 2$, p. 191.

Audition binauriculaire, vol. 28, no 2, p. 191.

Auditoire, vol. $28, \mathrm{n}^{\circ} 2$, p. 160.

AUPELF, vol. $28, n^{\circ} 1$, p. 93.

Auriculaire, vol. 28 , no 4 , p. 381 .

Auricule, vol. 28, no 4, p. 381.

Auriculo-, vol. 28, no 4, p. 381.

Authenticité, politique d', vol. $28, n^{\circ} 3$, p. 303.

Autopsie, vol. 28, no 2, p. 162.

Autoptique, vol. 28, no 2, p. 162.

Autorisation, vol. $28, \mathrm{n}^{\circ} 4$, p. 370

Autorité, vol. 28, no 4, p. 370.

Avoir le bien-être indigène, vol. $28, n^{\circ} 3$, p. 304.

Avoyage, vol. 28 , no 2 , p. 194.

Aza-, vol. 28, no $^{\circ}$, p. 169.

Aza-9 tétradecanoïque, vol. 28, no 2, p. 170.

Azote, vol. 28, n०2, p. 166.

Azul, vol. $28, \mathrm{n}^{\circ} 1$, p. 37.

Azur, vol. 28 , no 1 , p. 37.

Baignoire, vol. $28, n^{\circ} 3$, p. 303 .

Baladeur, vol. $28, n^{\circ} 4$, p. 400 .

Baladeur radio, vol. 28 , no 4 , p. 400.

Balafon, vol. 28 , no 3 , p. 303.

Balka, vol. 28, no 3, p. 303.

Balle perdue, vol. 28 , no 3 , p. 304 .

Ballon, vol. $28, n^{\circ} 3$, p. 304 .

Ballon stockeur, vol. 28, n $^{\circ} 3$, p. 289

Bamilékés, vol. 28, n $^{\circ} 1$, p. 25.

Bamoum, vol. $28, \mathrm{n}^{\circ} 1$, p. 25.

Bande-mère, vol. 28 , no 4 , p. 400 .

Bande passante, vol. 28, no 2, p. 191.

Bande promo, vol. $28, \mathrm{n}^{\circ} 4$, p. 400

Bande vidéo, vol. 28 , no 4 , p. 400 .

Bande vidéo promotionnelle, vol. $28, \mathrm{n}^{\circ} 4$, p. 400 .

Bandicon, vol. $28, n^{\circ} 3$, p. 304.

Bangala, vol. $28, n^{\circ} 3$, p. 304 .

Banque de terminologie du gouvernement canadien, vol. 28 , no 2 , p. 215 .

Banque de terminologie française, vol. $28, n^{\circ} 1$, p. 92.

Barreau, vol. $28, n^{\circ} 3$, p. 304

Barza, vol. 28, no 3, p. 304.

Base de campagne, vol. $28, n^{\circ} 4$, p. 400.

Base de données documentaires, vol. $28, n^{\circ} 2$, p. 218.

Base de données terminologiques, vol. $28, n^{\circ} 2$, p. 218.

Basse, vol. 28, no 2, p. 191.

Basse fréquence, vol. $28, n^{\circ} 2$, p. 191.

Bâtiment, vocabulaire contextuel du, vol. $28, \mathrm{n}^{\circ} 3$, p. 292.

Bâtonnet dentaire, vol. 28, no $^{\circ}$, p. 303.

Belle fidèle, la, vol. 28 , no 1, p. 44 . 
Beloteur, vol. 28, no 3, p. 303.

Bénigne, vol. $28, n^{\circ} 4$, p. 385.

Benzène, vol. 28, n $^{\circ} 2$, p. 166

Benzofuranne, vol. $28, \mathrm{n}^{\circ} 2$, p. 170.

Benzothiophène, vol. $28, \mathrm{n}^{\circ} 2$, p. 170.

Béref, vol. $28, \mathrm{n}^{\circ} 3$, p. 303

Bêta-adrénergique, vol. 28 , no 4 , p. 388.

Bêta-bloquant, vol. $28, n^{\circ} 4$, p. 388

Bêta-bloqueur, vol. $28, n^{\circ} 4$, p. 388 .

$\mathrm{Bi}-$, vol. $28, \mathrm{n}^{\circ} 2$, p. 170 ; vol. 28 , no 2 , p. 171

Bibliographie cursive, vol. 28, no $^{\circ} 2$, p. 219.

Bibliothèque de réseau, vol. $28, n^{\circ} 2$, p. 218.

Bibliothèque de section, vol. $28, \mathrm{n}^{\circ} 2, \mathrm{p} .218$

Bidane, vol. $28, n^{\circ} 3$, p. 303.

Bien-être indigène, avoir le, vol. $28, \mathrm{n}^{\circ} 3$, p. 304

Bière, vol. $28, n^{\circ} 2$, p. 179 ; vol. $28, n^{\circ} 2$, p. 184.

Bilinguisme océanien, vol. $28, \mathrm{n}^{\circ} 1, \mathrm{p} .17$.

Biloko, vol. 28, n० 3, p. 303.

Bini-, vol. $28, \mathrm{n}^{\circ} 2$, p. 171.

Bioxyde d'azote, vol. $28, n^{0} 3$, p. 298

Bis-, vol. 28, no $^{\circ}$, p. 170.

Bis-diméthyl-1',1'-propyl-5,5 méthyl-2 décane, vol. 28, no $^{\circ}$, p. 171 .

Bis(diméthyl-1,1 propyl)-5,5 méthyl-2 décane, vol. $28, n^{\circ} 2$, p. 171.

Bis(diméthylamico), vol. $28, \mathrm{n}^{\circ} 2$, p. 171.

Bisser, vol. $28, \mathrm{n}^{\circ} 3$, p. 304.

Bisseur, vol. 28, no 3, p. 304.

Bitter, vol. $28, n^{\circ} 2$, p. 185.

Blasphémateur, vol. $28, n^{\circ} 2$, p. 160.

Blasphématoire, vol. $28, n^{\circ} 2$, p. 160

Bleu, vol. 28, no $^{\circ}$, p. 303

Blinder, vol. 28 , no 3, p. 304.

Blocus, vol. $28, n^{\circ} 3$, p. 304

Bloquer, vol. 28, n $^{\circ} 3$, p. 303 ; vol. 28 , no 3 , p. 304 .

Bloqueur d'entrée du calcium, vol. 28, no 4 , p. 389.

Bloqueur des canaux du calcium, vol. $28, n^{\circ} 4$, p. 389 .

Bloqueur des récepteurs, vol. $28, n^{\circ} 4$, p. 388.

Bocfil, vol. $28, n^{\circ} 4$, p. 363.

Bois, vol. 28, no 3, p. 305.

Bois, caractéristique du, vol. $28, \mathrm{n}^{\circ} 3$, p. 304.

Bois courbe, vol. $28, n^{\circ} 4$, p. 358 .

Boisson alcoolique, vol. $28, n^{\circ} 2$, p. 178

Boisson alcoolisée, vol. $28, \mathrm{n}^{\circ} 2$, p. 178.

Boisson fermentée, vol. $28, \mathrm{n}^{\circ} 2$, p. 184.

Boisson spiritueuse, vol. 28, no 2, p. 184.

Boissons alcooliques, classification des, vol. 28 , $\mathrm{n}^{\circ} 2$, p. 184

Bondieu, vol. $28, n^{\circ} 2$, p. 199

Bordar, vol. $28, \mathrm{n}^{\circ} 1$, p. 37.

Bosser, vol. 28, no 3, p. 304

Boucle de régulation analogique indépendante, vol. $28, n^{\circ} 4$, p. 369.

Bourgmestre, vol. $28, n^{\circ} 3$, p. 303.

Bousiller, vol. $28, \mathrm{n}^{\circ} 3$, p. 304 .

Boyesse, vol. 28, no 3, p. 303.

Brandy, vol. 28, no 2, p. 185.

Bras, vol. 28, n $^{\circ} 2$, p. 193 ; vol. $28, n^{\circ} 2$, p. 194 ; vol. $28, n^{\circ} 2$, p. 196

Bras conventionnel, vol. 28, n 2, p. 191.

Bras pivotant, vol. 28, no $^{\circ}$, p. 191 .

Bras radial, vol. $28, \mathrm{n}^{\circ} 2$, p. 191.

Bras tangentiel, vol. $28, \mathrm{n}^{\circ} 2$, p. 191.

Bras-traverse, assemblage, vol. 28, n $^{\circ} 2$, p. 196.
Brésilien (traduction du), vol. 28, $\mathrm{n}^{\circ} 1$, p. 35.

Brevet, vol. 28, no 4, p. 371 .

Briquetage, vol. 28, no 3, p. 292.

Bromo-3 méthyl-2 hexanediol-1,6, vol. 28, $\mathrm{n}^{\mathrm{a}} 2$, p. 167.

Bromométhylhexanediol, vol. $28, \mathrm{n}^{\circ} 2$, p. 167.

Bruit, vol. $28, n^{\circ} 4$, p. 380.

Bruit d'airain, vol. $28, n^{\circ} 4$, p. 380

Bruit de bois, vol. $28, n^{\circ} 4$, p. 380 .

Bruit de canon, vol. 28 , no 4 , p. 380 .

Bruit de Korotkoff, vol. 28 , no 4 , p. 381

Bruit français, vol. $28, \mathrm{n}^{\circ} 4$, p. 380 .

Budgéter, vol. 28, no 3, p. 303.

Bureau linguistique, vol. $28, \mathrm{n}^{\circ} 1$, p. 22.

Business de la traduction, le, vol. $28, n^{\circ} 3$, p. 301.

Butadiène, vol. $28, \mathrm{n}^{\circ} 2$, p. 170 .

Butyrolactone, vol. $28, \mathrm{n}^{\circ} 2$, p. 169.

Bwana, vol. 28, no 3 , p. 303 .

Cabaret, vol. 28, no 3, p. 303.

Câbleur, vol. $28, n^{\circ} 4$, p. 400

Câblier, vol. $28, n^{\circ} 4$, p. 400 .

Câbliste, vol. 28, no 4 , p. 400

Cadre d'acier tubulaire, vol. $28, \mathrm{n}^{\circ} 4$, p. 363.

Cadre extensible, vol. $28, n^{\circ} 4$, p. 365 .

Cadre métallique, vol. $28, \mathrm{n}^{\circ} 2$, p. 192 ; vol. 28 , $n^{\circ} 4$, p. 364 .

Cadreur, vol. $28, n^{\circ} 4$, p. 400.

Cahier des charges, vol. $28, n^{\circ} 4$, p. 375.

Caille, vol. $28, n^{\circ} 3$, p. 304 .

Cailler, vol. $28, n^{\circ} 3$, p. 304

Caillerie, vol. $28, n^{\circ} 3$, p. 304.

Calage, vol. $28, n^{\circ} 3$, p. 289.

Calculateur, vol. $28, n^{\circ} 2$, p. 160

Calculateur industriel, vol. $28, n^{\circ} 4$, p. 369.

Caller, vol. $28, n^{\circ} 3$, p. 304.

Caloduc, vol. $28, \mathrm{n}^{\circ} 3$, p. 289.

Calomniateur, vol. $28, \mathrm{n}^{\mathrm{0}} 2$, p. 160.

Calque, vol. $28, n^{\circ} 3$, p. 303 .

Cameroun, vol. $28, n^{\circ} 1$, p. 20

Cameroun occidental anglophone, vol. $28, n^{\circ} 1$, p. 20.

Cameroun oriental francophone, vol. $28, \mathrm{n}^{\circ} 1$, p. 20.

Camerounais anglophone, vol. $28, n^{\circ} 1$, p. 25.

Camerounais francophone, vol. $28, n^{\circ} 1$, p. 25.

Campagne de recouvrement des impôts, vol. 28 , no 1, p. 23.

CAN, vol. $28, n^{\circ} 4$, p. 369

Canal calcique, vol. $28, n^{\circ} 4$, p. 389

Capitaine, vol. $28, \mathrm{n}^{\circ} 3$, p. 303.

Captage, vol. $28, n^{\circ} 3$, p. 289.

Captation, vol. 28, n $^{\circ} 3$, p. 289

Capteur à air, vol. 28, no 3 , p. 289

Capteur à concentration, vol. $28, n^{\circ} 3$, p. 289.

Capteur à eau, vol. $28, n^{\circ} 3$, p. 289

Capteur à fluide liquide, vol. $28, n^{\circ} 3$, p. 289.

Capteur de mesure, vol. $28, \mathrm{n}^{\circ} 4$, p. 367 .

Capteur plan, vol. $28, n^{\circ} 3$, p. 289.

Capteur solaire, vol. $28, \mathrm{n}^{\mathrm{0}} 3$, p. 289

Capteur thermique, vol. 28, no $^{\circ} 3$, p. 289.

Captiverie, vol. $28, n^{\circ} 3$, p. 303.

Caractéristique du bois, vol. 28, no 3, p. 304.

Carbazole, vol. 28, no 2, p. 170. 
Carbone, vol. 28, no 2, p. 166.

Carton, vol. 28 , no 4 , p. 400 .

Carton publicitaire, vol. $28, n^{\circ} 4$, p. 400

Case de santé, vol. 28, n $^{\circ} 3$, p. 303 .

Case vide, vol. $28, n^{\circ} 4$, p. 393

Casseur, vol. $28, n^{\circ} 3$, p. 303

Cassis, vol. $28, \mathrm{n}^{\circ} 2, \mathrm{p} .185$

Céfé, vol. $28, n^{\circ} 3$, p. 304

Cellule, vol. 28, no 2, p. 192.

Censeur, vol. 28, no $^{\circ}$, p. 160.

Centre d'activités langagières, vol. $28, \mathrm{n}^{\circ} 1$, p. 94.

Cercle de Prague, vol. 28, no 2, p. 136.

Céspedes, Luis de, vol. $28, n^{\circ} 4$, p. 354.

C'est pourquoi, vol. $28, n^{\circ} 3$, p. 268.

Cétone, vol. $28, n^{\circ} 2$, p. 168.

Chaîne audiophonique, vol. $28, \mathrm{n}^{\circ} 2$, p. 191

Chaîne carbonée, vol. $28, n^{\circ} 2$, p. 166 ; vol. 28 , $\mathrm{n}^{\circ} 2$, p. 167.

Chaine latérale, vol. $28, \mathrm{n}^{\circ} 2$, p. 166 ; vol. $28, \mathrm{n}^{\circ} 2$, p. 170 ; vol. $28, n^{\circ} 2$, p. 171

Chaîne principale, vol. 28, no $^{\circ}$, p. 171.

Chaînon, vol. 28, no 2, p. 171.

Chamar, vol. $28, \mathrm{n}^{\circ} 1$, p. 37.

Champ auditif, vol. $28, n^{\circ} 2$, p. 191.

Champ d'étude, vol. $28, n^{\circ} 3$, p. 280

Champ sémantique, vol. $28, n^{\circ} 4$, p. 329 ; vol. 28 , $\mathrm{n}^{\circ} 4$, p. 392.

Champion du polyglottisme, vol. $28, n^{\circ} 4$, p. 340

Changement de code, vol. $28, \mathrm{n}^{\circ} 2$, p. 133

Chanson populaire, vol. $28, \mathrm{n}^{\circ} 1$, p. 41 .

Chantournement, vol. $28, \mathrm{n}^{\circ} 2$, p. 196.

Chantournement fermé, vol. $28, n^{\circ} 4$, p. 362.

Chaperon, vol. $28, n^{\circ} 4$, p. 361 .

Charbon, gazéification du, vol. 28, no $^{\circ}$, p. 297.

Charbons, classification des, vol. $28, n^{\circ} 3$, p. 298.

Chauffage d'appoint, vol. $28, \mathrm{n}^{\circ} 3$, p. 289.

Chauffage solaire, vol. $28, \mathrm{n}^{\circ} 3$, p. 289.

Chauffage solaire domestique, vol. $28, n^{\circ} 3$, p. 283.

Chauffage solaire, lexique, vol. $28, n^{\circ} 3$, p. 288 .

Chef-cir, vol. $28, n^{\circ} 3$, p. 304

Chef de bureau, vol. $28, n^{\circ} 1$, p. 23.

Chefferie, conflit de, vol. $28, n^{\circ} 1$, p. 23

Chegar, vol. $28, \mathrm{n}^{\circ} 1$, p. 37

Chemin, donner du, vol. 28, no 2, p. 194.

Cheminement du traducteur, vol. $28, n^{\circ} 4$, p. 325

Cheminement orateur-délégués, vol. $28, n^{\circ} 3$, p. 238.

Chevauchement, vol. $28, n^{\circ} 1$, p. 49.

Chevrette, vol. 28, no $^{\circ}$, p. 194

Chier, vol. $28, \mathrm{n}^{\circ} 3$, p. 303

Chiffre binaire, vol. $28, n^{\circ} 4$, p. 369 .

Chimie générale, vol. 28, n $^{\circ} 3$, p. 305 .

Chimie industrielle, vol. 28, no $^{\circ} 3$, p. 305

Chimie organique, vol. $28, n^{\circ} 2$, p. 166.

Chlore, vol. 28, no 2, p. 166.

Chlorobenzène, vol. $28, \mathrm{n}^{\circ} 2$, p. 166

Chlorure, vol. $28, \mathrm{n}^{\circ} 2$, p. 168 .

Chlorure de phényl-mercure, vol. $28, \mathrm{n}^{\circ} 2, \mathrm{p} .168$.

Cidre, vol. $28, \mathrm{n}^{\circ} 2$, p. 179 ; vol. $28, \mathrm{n}^{\circ} 2$, p. 184.

Ciné-parc, vol. $28, n^{\circ} 4$, p. 400 .

Circuit de la communication, vol. $28, n^{0} 2$, p. 206.

Circulation pulmonaire, vol. $28, n^{\circ} 4$, p. 384 .

Clairet, vol. $28, \mathrm{n}^{\circ} 2$, p. 185 .

Classage, vol. $28, n^{\circ} 3$, p. 305.

Classification des boissons alcooliques, vol. 28 , $n^{\circ} 2$, p. 184.
Classification des charbons, vol. $28, \mathrm{n}^{\circ} 3$, p. 298.

Classification des fuels, vol. $28, \mathrm{n}^{\circ} 3$, p. 298.

Classification des gaz, vol. $28, \mathrm{n}^{\circ} 3$, p. 298.

Classification, système de, vol. $28, \mathrm{n}^{\mathrm{O}} 4$, p. 395 .

Clause, vol. $28, n^{\circ} 4$, p. 371 ; vol. 28 , no 4 , p. 375

Clavier, vol. 28, no $^{\circ}$, p. 299.

Clavier à cannes, vol. $28, \mathrm{n}^{\circ} 3$, p. 299.

Clavier à poussoir, vol. $28, n^{\circ} 3$, p. 299.

Clé, vol. $28, n^{\circ} 2$, p. 193 ; vol. 28 , n 2, p. 196.

Clef, vol. $28, n^{\circ} 2$, p. 196.

CMR, vol. 28, n $^{\circ} 1$, p. 23.

CNA, vol. $28, n^{\circ} 4$, p. 369

Code oral, vol. $28, \mathrm{n}^{\circ} 1$, p. 50.

Code temporel, vol. 28 , no 4 , p. 400 .

Codeur analogique numérique, vol. $28, n^{\circ} 4$, p. 369.

Cognac, vol. 28, n $^{\circ} 2$, p. 185

Cohyponyme, vol. $28, n^{\circ} 4$, p. 395.

Collaborer au régime, vol. $28, n^{\circ} 4$, p. 387.

Collecteur, vol. $28, \mathrm{n}^{\circ} 2$, p. 160.

Collectif, vol. $28, n^{\circ} 2$, p. 160.

Collection, vol. $28, n^{\circ} 2$, p. 160.

Collègue passif, vol. $28, \mathrm{n}^{\circ} 3$, p. 239.

Colloque, vol. $28, \mathrm{n}^{\circ} 3$, p. 279.

Colloque de traduction, vol. $28, n^{\circ} 4$, p. 432.

Colloque franco-britannique, vol. $28, n^{\circ} 4$, p. 432.

Colloque : Traduction et qualité de langue, vol. $28, n^{\circ} 2$, p. 220

Coloration, vol. $28, \mathrm{n}^{\circ} 2$, p. 191.

Coloration du signe, vol. $28, n^{\circ} 1$, p. 55.

COM, vol. $28, n^{\circ} 2$, p. 219

Combinaison organo-métallique, vol. $28, \mathrm{n}^{\circ} 2$, p. 168.

Combinatoire, vol. $28, \mathrm{n}^{\circ} 2$, p. 160.

Comité, vol. $28, n^{\circ} 4$, p. 371 ; vol. 28 , no 4 , p. 372 .

Comité international des études françaises, vol. 28 , no 1, p. 93.

Commande d'équilibre, vol. 28, nº 2, p. 191.

Commanditaire, vol. $28, n^{\circ} 4$, p. 401 .

Commanditer, vol. 28, $\mathrm{n}^{\circ} 4$, p. 401.

Commentateur, vol. $28, n^{\circ} 2$, p. 160.

Commis aux expéditions, vol. $28, \mathrm{n}^{\circ} 1, \mathrm{p} .23$.

Commis principal, vol, $28, \mathrm{n}^{\circ} 1$, p. 23.

Commissaire, vol. $28, \mathrm{n}^{\circ} 4$, p. 372.

Commission, vol. $28, n^{\circ} 4$, p. 371 ; vol. $28, n^{\circ} 4$, p. 372.

Commission de nomenclature pour la chimie organique, vol. $28, \mathrm{n}^{\circ} 2$, p. 166.

Commission départementale, vol. 28, no 4, p. 376.

Commission du Pacifique du Sud, vol. 28, $\mathrm{n}^{\circ} 1$, p. 19.

Communauté urbaine, vol. $28, \mathrm{n}^{0} 4$, p. 376

Commune, vol. $28, n^{\circ} 4$, p. 376.

Commune de plein exercice, vol. $28, n^{\circ} 1$, p. 23.

Commune mixte rurale, vol. $28, \mathrm{n}^{\circ} 1$, p. 23.

Communication, circuit de la, vol. 28, no $^{\circ}$, p. 206.

Communication, condition physique de la, vol. 28 , no 3, p. 237.

Comparateur, vol. $28, n^{\circ} 4$, p. 367.

Comparatif, vol. 28 , no 2 , p. 160.

Compétence-performance, vol. 28, n० 2, p. 142.

Compliance, vol. $28, n^{\circ} 4$, p. 387.

Composé aliphatique, vol. $28, n^{\circ} 2$, p. 169

Composé cyclique, vol. $28, \mathrm{n}^{\circ} \mathrm{2}, \mathrm{p} .169$.

Composé fondamental, vol. $28, \mathrm{n}^{\circ} 2$, p. 167. 
Composés organiques, nomenclature des, vol. 28 , $n^{0} 2$, p. 166.

Composition, vol. $28, n^{\circ} 3$, p. $299 ;$ vol. $28, n^{\circ} 3$ p. 303.

Compréhension, vol. $28, n^{\circ} 2$, p. 133 ; vol. $28, n^{\circ} 2$, p. 206.

Comptabilité, vol. $28, n^{0} 4$, p. 413.

Compte rendu, vol. $28, \mathrm{n}^{\circ} 2$, p. 176 .

Comptine enfantine, vol. $28, \mathrm{n}^{\circ} 1$, p. 41.

Condition physique de la communication, vol. 28 , $n^{\circ} 3$, p. 237.

Conducteur, vol. 28, $\mathrm{n}^{\circ} 4$, p. 401.

Conflit de chefferie, vol. $28, \mathrm{n}^{\circ} 1$, p. 23

Congrès de la SILF, vol. $28, n^{\circ} 4$, p. 431.

Connotation, vol. $28, n^{\circ} 1$, p. 53 ; vol. $28, n^{\circ} 2$, p. 133.

Connotation académique, vol. $28, \mathrm{n}^{\circ} 1$, p. 55 .

Connotation archaïque, vol. $28, \mathrm{n}^{\circ} 1$, p. 55 .

Connotation argotique, vol. $28, \mathrm{n}^{\circ} 1, \mathrm{p} .55$.

Connotation enfantine, vol. $28, n^{\circ} 1$, p. 55

Connotation étrangère, vol. $28, n^{\circ} 1$, p. 55

Connotation familière, vol. $28, n^{\circ} 1$, p. 55 .

Connotation ironique, vol. $28, \mathrm{n}^{\circ} 1$, p. 55 .

Connotation provinciale, vol. $28, \mathrm{n}^{\mathrm{0}} 1, \mathrm{p} .55$.

Connotation rustique, vol. $28, \mathrm{n}^{\circ} 1$, p. 55.

Connotation savante, vol. $28, n^{\circ} 1$, p. 55.

Connotation technique, vol. $28, n^{\circ} 1$, p. 55.

Connotation vulgaire, vol. $28, \mathrm{n}^{\circ} 1$, p. 55 .

Conscience acadienne, vol. $28, n^{\circ} 4$, p. 352.

Consécutif, vol. $28, n^{\circ} 2$, p. 160.

Conseil, vol. $28, n^{\circ} 4$, p. 372 .

Considération traductionologique, vol. $28, \mathrm{n}^{\circ} 2$, p. 131.

Constitution d'un corpus, vol. $28, n^{\circ} 3$, p. 242.

Construction, vol. $28, \mathrm{n}^{\circ} 2$, p. 142.

Conte, vol. $28, n^{\circ} 1$, p. 36

Contenu informationnel, vol. $28, \mathrm{n}^{0} 3$, p. 236.

Contexte, vol. $28, n^{\circ} 2$, p. 142 ; vol. $28, n^{\circ} 3$, p. 305.

Contexte circonstanciel, vol. $28, \mathrm{n}^{\circ} 1$, p. 53.

Contexte cognitif, vol. $28, n^{\circ} 2$, p. 205.

Contexte de situation, vol. $28, \mathrm{n}^{\circ} 2$, p. 130 .

Contexte situationnel, vol. $28, \mathrm{n}^{\circ} 2$, p. 137 ; vol. $28, n^{\circ} 2$, p. 141 .

Contexte verbal, vol. $28, n^{\circ} 2$, p. 205

Contour, vol. $28, n^{\circ} 4$, p. 401

Contour du signifié, vol. $28, \mathrm{n}^{\circ} 1$, p. 49.

Contrôle, vol. 28, no $^{\circ}$, p. 23 .

Contrôler l'hypertension, vol. $28, n^{\circ} 4$, p. 386.

Conversion héliothermique, vol. $28, n^{\circ} 3$, p. 289.

Conversion photothermique, vol. $28, n^{\circ} 3$, p. 289

Conversion thermique, vol. $28, \mathrm{n}^{\circ} 3$, p. 289.

Convertisseur analogique numérique, vol. $28, n^{\circ} 4$, Dans les coulisses de la traduction, vol. $28, n^{\circ} 4$, p. 369.

Convertisseur numérique-analogique, vol. $28, n^{\circ} 4$, p. 369.

Convulsivant, vol. $28, n^{\circ} 4$, p. 388

Coopérative, vol. $28, n^{\circ} 1$, p. 23.

Copie d'antenne, vol. $28, \mathrm{n}^{\circ} 4$, p. 401.

Copie de diffusion, vol. 28, no 4 , p. 401.

Copion, vol. $28, n^{\circ} 3$, p. 304 .

Coran, vol. $28, n^{\circ} 3$, p. 303.

Corde de tension, vol. 28 , no 2 , p. 193 ; vol. 28 , $\mathrm{n}^{\circ} 2$, p. 196.

Cordon, vol. 28, no 3, p. 292.
Corps de doctrine traductionnelle, vol. $28, n^{\circ} 4$, p. 332.

Corps insaturé, vol. $28, \mathrm{n}^{\circ} 2$, p. 168.

Corpus, constitution d'un, vol. $28, \mathrm{n}^{\circ} 3$, p. 242.

Correctif, vol. 28, $\mathrm{n}^{\circ} 2$, p. 162.

Correction, vol. $28, n^{\circ} 2$, p. 162.

Correr, vol. 28, no $^{\circ}$, p. 37.

Correspondance de forme, vol. $28, n^{\circ} 2$, p. 133

Correspondance de sens, vol. $28, n^{\circ} 2, p .133$.

Cou-plié, vol. 28, n $^{\circ} 3$, p. 303.

Couche antiréfléchissante, vol. 28, no $^{\circ} 3$, p. 289.

Couche antireflet, vol. $28, n^{\circ} 3$, p. 289.

Couche sélective, vol. $28, n^{\circ} 3$, p. 289.

Couiller, vol. 28, n $^{\circ} 3$, p. 304.

Coulisse de la traduction, vol. $28, n^{\circ} 4$, p. 334.

Couper sec, vol. $28, n^{\circ} 4$, p. 401.

Coupure de capillarité, vol. 28, no 3, p. 292

Courtiser, vol. $28, n^{\circ} 3$, p. 303.

Cousin à plaisanterie, vol. $28, n^{\circ} 3$, p. 303.

Couverture, vol. $28, n^{\circ} 3$, p. 289.

Cow-boy, vol. $28, n^{\circ} 3$, p. 304.

CPE, vol. $28, \mathrm{n}^{\circ} 1$, p. 23.

CPS, vol. $28, n^{\circ} 1$, p. 19.

Crapule, vol. $28, \mathrm{n}^{\circ} 3$, p. 303.

Crayonné, vol. $28, n^{\circ} 4$, p. 401

Créateur, vol. $28, \mathrm{n}^{\circ} 2$, p. 160 .

Création, vol. $28, n^{\circ} 2$, p. 160.

Création du merveilleux, vol. $28, \mathrm{n}^{\circ} 1$, p. 40.

Création, mode de, vol. 28 , no 3 , p. 299.

Création néologique stylistique, vol. $28, n^{\circ} 3$, p. 299.

Créativité lexicale, la, vol. 28, no 3, p. 299.

Cristal, vol. 28, no 2, p. 184.

Critère de dispersion géographique, vol. $28, \mathrm{n}^{\circ} 3$, p. 303.

Critère d'exclusion, vol $28, n^{\circ} 3$, p. 280.

Critère d'intégration, vol. $28, n^{\circ} 3$, p. 303

Croc, donner du, vol. 28, n², p. 195.

Crochet, donner du, vol. $28, \mathrm{n}^{\circ} 2$, p. 195.

Crosse aortique, vol. $28, \mathrm{n}^{\circ} 4$, p. 383.

Crossette, vol. $28, n^{\circ} 2$, p. 193 ; vol. $28, n^{\circ} 2$, p. 196.

CS, vol. $28, n^{\circ} 1$, p. 52 .

Cuer, vol. $28, n^{\circ} 3$, p. 304.

Cul, vol. 28, nº 3 , p. 304.

Cultivateur, vol. $28, \mathrm{n}^{\circ} 2$, p. 160

Cuve d'accumulation, vol. 28, no 3, p. 289.

Cuve de stockage, vol. 28, no $^{\circ}$, p. 290.

Cycle, vol. 28, no $^{\circ}$, p. 170 .

$$
\text { p. } 334 .
$$

DB, vol. 28, no 2, p. 191.

DD, vol. $28, n^{\circ} 2$, p. 218

De la pratique à la théorie, vol. $28, n^{\circ} 4$, p. 323 .

Débat, vol. 28, n० 2, p. 176.

Débit, vol. $28, n^{\circ} 2$, p. 196.

Débit calorifique, vol. 28 , no 3 , p. 299.

Débit premier, vol. 28, $\mathrm{n}^{\circ} 2$, p. 196.

Débit second, vol. $28, \mathrm{n}^{\circ} 2$, p. 196.

Débiter, vol. 28, n $^{\circ} 4$, p. 359.

Débordement, vol. $28, \mathrm{n}^{\circ} 4$, p. 401.

Décéder, vol. $28, \mathrm{n}^{\circ} 1$, p. 53 .
Corps noir, vol. $28, n^{\circ} 3$, p. 289. 
Décélération, vol. $28, \mathrm{n}^{\circ} 2$, p. 161.

Décentrement, vol. 28 , no 1, p. 45 .

Déchirure, stratégie de la, vol. $28, n^{\circ} 2$, p. 208.

Décibel, vol. $28, \mathrm{n}^{\circ} 2$, p. 191.

Décision, vol. $28, n^{\circ} 2$, p. 176

Déclencheur de précipitation, vol. $28, \mathrm{n}^{\circ} 3$, p. 255

Décodage, vol. $28, \mathrm{n}^{\circ} 2$, p. 133.

Décollement, vol. 28 , no 3, p. 294.

Découpage, vol. $28, n^{\circ} 4$, p. 364.

Découpage de la réalité, vol. $28, n^{\circ} 2$, p. 133.

Découpage du réel, vol. $28, n^{\circ} 1$, p. 51 .

Décret, vol. 28 , no 4 , p. 370

Décrocheur, vol. $28, n^{\circ} 4$, p. 387.

Dédicatoire, vol. $28, n^{\circ} 2$, p. 160 .

DEF, vol. $28, n^{\circ} 1$, p. 93.

Défaut d'homogénéité dans le repérage, vol. 28 , $\mathrm{n}^{\circ} 3,-\mathrm{p} .280$.

Défectionnaire, vol. $28, n^{\circ} 4$, p. 387.

Défenseur de Macpherson, vol. 28, nª 4, p. 335.

Déficit auditif, vol. $28, n^{\circ} 2$, p. 191

Définition en compréhension, vol. $28, n^{\circ} 3$, p. 281

Définition formelle, vol. $28, n^{\circ} 3$, p. 280.

Déflation, vol. $28, \mathrm{n}^{\circ} 2$, p. 161 .

Déflexion, vol. $28, \mathrm{n}^{\circ} 2$, p. 161 .

Déforcer, vol. 28, no 3, p. 303.

Déglutination, vol. $28, \mathrm{n}^{\mathrm{o}} 2$, p. 161.

Degré-jour, vol. $28, \mathrm{n}^{\circ} 3$, p. 290.

Déhydro-, vol. $28, \mathrm{n}^{\circ} 2$, p. 168.

Délégation, vol. $28, n^{\circ} 4$, p. 371 .

Délibération, vol. $28, n^{\circ} 2$, p. 176.

Demander la route, vol. $28, \mathrm{n}^{\circ} 3$, p. 303.

Démarche, vol. $28, \mathrm{n}^{\circ} 2$, p. 175.

Démerder, vol. $28, n^{\circ} 3$, p. 303

Demeurer, vol. $28, \mathrm{n}^{\circ} 1$, p. 37.

Déni de justice, vol. $28, \mathrm{n}^{\circ} 2$, p. 213.

Dénomination, vol. 28, no $^{\circ} 3$, p. 283.

Dénotation, vol. $28, \mathrm{n}^{\circ} 1, \mathrm{p} .53$.

Dent "américaine ", vol. $28, \mathrm{n}^{\circ} 2$, p. 195

Dent biseautée, vol. $28, n^{\circ} 2$, p. 195.

Dent de loup, vol. 28, no 2, p. 195.

Dent double, vol. $28, \mathrm{n}^{\circ} 2$, p. 195.

Dent isocèle, vol. $28, n^{\circ} 2$, p. 195 .

Dent isocèle à gencives, vol. $28, \mathrm{n}^{\circ} 2$, p. 195.

Dent rabotante, vol. $28, \mathrm{n}^{\circ} 2$, p. 195.

Dents, nombre de, vol. $28, \mathrm{n}^{\circ} 2$, p. 196.

Denture, vol. $28, n^{\circ} 2$, p. 195.

Denture fine, vol. $28, n^{\circ} 4$, p. 364.

Denture triangulaire couchée, vol. $28, \mathrm{n}^{\circ} 2$, p. 195 ; vol. $28, n^{\circ} 4$, p. 359.

Denture triangulaire isocèle, vol. $28, n^{\circ} 4$, p. 358 .

Département, vol. $28, \mathrm{n}^{\circ} 3$, p. 260.

Département d'études françaises, vol. $28, \mathrm{n}^{\circ} 1$, p. 93.

Déprédateur, vol. 28 , no 2, p. 160.

Déprédation, vol. $28, \mathrm{n}^{\circ} 2$, p. 160.

Député, vol. $28, \mathrm{n}^{\circ} 1$, p. 23.

Dérivation, vol. $28, \mathrm{n}^{\circ} 3$, p. 299 ; vol. $28, \mathrm{n}^{\circ} 3$, p. 303.

Dérivation lexicale, vol. 28, no $^{\circ} 3$, p. 299.

Dérivation, théorie de la, vol. $28, n^{\circ} 4$, p. 331

Dérober, vol. $28, \mathrm{n}^{\circ} 1$, p. 37.

Désarmer, vol. $28, n^{\circ} 3$, p. 303.

Descer, vol. $28, n^{\circ} 1$, p. 37.

Descripteur, vol. 28 , no 2 , p. 218

Description linguistique, vol. $28, n^{\circ} 2$, p. 141.

Description vs prescription, vol. $28, n^{\circ} 2$, p. 143.
Désinence verbale, vol. $28, \mathrm{n}^{0} 1$, p. 50

Désorption, vol. $28, \mathrm{n}^{\circ} 2$, p. 161 .

Désoxy-, vol. $28, n^{\circ} 2$, p. 168.

Désoxyribose, vol. $28, n^{\circ} 2$, p. 169.

Dessin figuratif d'équipements, vol. $28, n^{\circ} 3$, p. 305.

Dessinateur, vol. $28, n^{\circ} 2$, p. 160

Désynchronisation ponctuelle, vol. $28, n^{0} 4$, p. 401.

Déterminant, vol. $28, n^{\circ} 3$, p. 299.

Déterminatif, vol. $28, \mathrm{n}^{\circ} 1$, p. 39.

Détracteur de Macpherson, vol. 28, no 4, p. 335.

Deuxième bruit du coeur, vol. $28, n^{\circ} 4$, p. 380 .

Dextroposition de l'aorte, vol. $28, n^{\circ} 4$, p. 384 .

DGTD, vol. $28, n^{\circ} 2$, p. 218.

Di-, vol. $28, n^{\circ} 2$, p. 170

Diaphonie, vol. 28, no $^{\circ}$, p. 191.

Diastole, vol. $28, n^{\circ} 4$, p. 377 .

Dibiterie, vol. 28, no 3, p. 303.

Dichloréthane, vol. $28, \mathrm{n}^{\circ} 2$, p. 168.

Dichloro-1,1 chloro-1'-éthyl-4 chloro-1"-propyl-7 dodécane, vol. $28, \mathrm{n}^{\circ} 2$, p. 171 .

Dichloro-1,1 (chloro-1 éthyl)-4(chloro-1 propyl)-7 dodécane, vol. $28, \mathrm{n}^{\circ} 2$, p. 171 .

Dichlorure d'éthylène, vol. $28, \mathrm{n}^{\circ} 2$, p. 168 .

Dichotomie pratique/théorie, vol. $28, \mathrm{n}^{\circ} 2$, p. 207.

Dictée, vol. $28, \mathrm{n}^{\circ} 2$, p. 162.

Diction, vol. $28, n^{\circ} 2$, p. 162.

Dictionnaire automatique, vol. $28, \mathrm{n}^{0} 2$, p. 215 ; vol. $28, n^{\circ} 3$, p. 280.

Dictionnaire de définitions formelles, vol. $28, \mathrm{n}^{\circ} 3$, p. 305.

Dictionnaire de la comptabilité, vol. $28, \mathrm{n}^{\circ} 4$, p. 413.

Dictionnaire de la linguistique, vol. $28, n^{\circ} 4$, p. 410 .

Dictionnaire de l'industrie du gaz, vol. $28, \mathrm{n}^{\circ} 3$, p. 297.

Dictionnaire du génie automobile, vol. $28, \mathrm{n}^{0} 4$ p. 412.

Dicton, vol. $28, n^{\circ} 1$, p. 41 .

Didacticiels, traduction des, vol. $28, \mathrm{n}^{\circ} 1$, p. 94

Diffamation, vol. $28, \mathrm{n}^{\circ} 2$, p. 162.

Diffamatoire, vol. $28, \mathrm{n}^{\circ} 2$, p. 162

Différence culturelle, vol. $28, \mathrm{n}^{\circ} 1$, p. 25 ; vol. 28 , $n^{\circ} 3$, p. 312 .

Différence linguistique, vol. $28, \mathrm{n}^{\circ} 3$, p. 312.

Différence socio-linguistique, vol. $28, \mathrm{n}^{\circ} 1$, p. 25 .

Diffusion des idées, instrument de, vol. $28, n^{\circ} 4$, p. 343.

Dilatation, vol. 28, no 3, p. 293.

Diligence, vol. $28, \mathrm{n}^{\circ} 2$, p. 175 .

Dipsomane, vol. $28, n^{\circ} 2$, p. 180

Dipsomanie, vol. $28, n^{\circ} 2$, p. 180.

Direction, vol. $28, n^{\circ} 2$, p. 162.

Direction de la documentation, vol. $28, \mathrm{n}^{\mathrm{O}} 2$, p. 218.

Direction des services linguistiques, vol. $28, \mathrm{n}^{\circ} 1$, p. 22.

Direction générale de la terminologie et de la documentation, vol. $28, n^{\circ} 2$, p. 218 .

Directoire, vol. 28, no 2, p. 162.

Discours de bienvenue, vol. $28, n^{\circ} 3$, p. 237

Discours de remerciement, vol. $28, n^{\circ} 3$, p. 237.

Discours du Président de la République, vol. 28, $n^{\circ} 1$, p. 21

Discours raconté, vol. $28, n^{\circ} 3$, p. 250. 
Discours rapporté, vol. 28, no 3, p. 250.

Discours scientifique, vol. $28, n^{\circ} 3$, p. 237

Discours technique, vol. $28, n^{\circ} 3$, p. 237.

Discours transposé, vol. 28 , no 3 , p. 250.

Discours, unité du, vol. $28, \mathrm{n}^{\circ} 2$, p. 143.

Disparité, vol. $28, n^{\circ} 3$, p. 282

Dispensateur, vol. $28, n^{\circ} 2$, p. 160

Dispersion, vol. 28 , no 3 , p. 266.

Dispersion géographique, critère de, vol. $28, \mathrm{n}^{\circ} 3$, p. 303.

Disposition des enceintes acoustiques, vol. 28 , $n^{\circ} 2$, p. 191

Disque audionumérique, vol. $28, n^{\circ} 4$, p. 401

Distillat non neutre, vol. $28, \mathrm{n}^{\circ} 2$, p. 185 .

Distillation, vol. $28, n^{\circ} 2$, p. 179 .

Distorsion, taux de, vol. 28 , no 2 , p. 192.

Distribution artistique, vol. $28, n^{\circ} 4$, p. 401 .

Division des services techniques, vol. $28, \mathrm{n}^{\circ} 2$, p. 219.

Division du réseau de bibliothèques, vol. $28, \mathrm{n}^{\circ} 2$, p. 219.

Do-, vol. 28 , no 2 , p. 170

Doctrine traductionnelle, corps de, vol. $28, n^{\circ} 4$, p. 332.

Document, vol. 28, n $^{\circ} 4$, p. 401.

Documentation, vol. $28, \mathrm{n}^{\circ} 2$, p. 217.

Documentation, Direction de la, vol. $28, \mathrm{n}^{\circ} 2$, p. 218.

Documentation, organisation de la, vol. $28, n^{\circ} 2$, p. 217.

Documentation pour la traduction et la terminologie, vol. $28, n^{\circ} 2$, p. 219.

Dolo doux, vol. $28, \mathrm{n}^{\circ} 3$, p. 303 .

Domaine, articulation du, vol. $28, n^{\circ} 3$, p. 280

Domaine de pression, vol. $28, n^{\circ} 3$, p. 298.

Données documentaires, base de, vol. $28, \mathrm{n}^{\circ} 2$, p. 218.

Données, exploitation des, vol. $28, n^{\circ} 3$, p. 242.

Données terminologiques, base de, vol. $28, n^{\circ} 2$, p. 218.

Donner de la voie, vol. $28, \mathrm{n}^{\circ} 2$, p. 194

Donner du chemin, vol. 28, n $^{\circ} 2$, p. 194 .

Donner du croc, vol. 28, no 2, p. 195.

Donner du crochet, vol. 28 , no 2 , p. 195.

Donner du passage, vol. $28, n^{\circ} 2$, p. 194.

Dotriacontahecta, vol. $28, \mathrm{n}^{\circ} 2$, p. 170 .

DOTT, vol. $28, \mathrm{n}^{\circ} 2$, p. 219.

Double ponte, vol. $28, \mathrm{n}^{\circ} 3$, p. 258.

Doubleur, vol. $28, n^{\circ} 3$, p. 303 ; vol. $28, n^{\circ} 3$, p. 304.

Doubleuse, vol. $28, n^{\circ} 3$, p. 304

Douchière, vol. $28, n^{\circ} 3$, p. 303.

Doué de sens, vol. 28 , no 2 , p. 140

Droit public, vol. 28, n $^{\circ} 4$, p. 369

Eau-de-vie composée, vol. 28, no 2, p. 185.

Eau-de-vie naturelle, vol. $28, n^{\circ} 2$, p. 185.

Écaillement, vol. 28, n $^{\circ} 3$, p. 294.

Écart supérieur aux tolérances, vol. $28, n^{\circ} 4$, p. 367

Échangeur d'ions, vol. $28, \mathrm{n}^{\circ} 3$, p. 305.

Échantillonnage, vol. $28, n^{\circ} 4$, p. 369 .

Échelle ordinale à 7 paliers, vol. $28, n^{\circ} 3$, p. 241

Éclatement, vol. $28, n^{\circ} 3$, p. 294
Éclipse, vol. 28, n², p. 162.

Écliptique, vol. 28, no 2, p. 162.

École danoise, vol. 28, n०2, p. 136.

École supérieure d'interprètes et de traducteurs, vol. 28 , n० 1 , p. 91 .

École transformationnelle, vol. $28, n^{\circ} 2$, p. 136

Écorçage, vol. $28, n^{\circ} 3$, p. 305

Écran de contrôle, vol. $28, n^{\circ} 4$, p. 401 .

Ecran pare-vapeur, vol. 28 , no 3, p. 294

Écran témoin, vol. 28 , no 4, p. 401 .

Écriture, vol. $28, n^{\circ} 2$, p. 138.

Effet de membrane, vol. $28, n^{\circ} 4$, p. 389.

Effet de salle, vol. 28, no 2, p. 191.

Effet de sens, vol. $28, n^{\circ} 1$, p. 52 .

Effet de serre, vol. 28 , n० 3, p. 290

Effet du sens, vol. $28, n^{\circ} 1$, p. 52.

Effet sélectif, vol. 28, no $^{\circ}$, p. 290.

Effet stabilisant de membrane, vol. $28, n^{\circ} 4$ p. 389 .

Effet stabilisateur des membranes, vol. $28, n^{\circ} 4$, p. 389.

Effet stéréophonique, vol. $28, \mathrm{n}^{\circ} 2$, p. 191.

Efficacité du message, vol. 28, n $^{\circ} 3$, p. 238.

Efflorescence, vol. $28, n^{\circ} 3$, p. 293.

Égalisateur, vol. $28, \mathrm{n}^{\circ} 4$, p. 401 .

Egussi, vol. 28, no 1, p. 25

Électrodialyse, vol. $28, n^{\circ} 3$, p. 305.

Elément différentiel, vol. $28, n^{\circ} 1$, p. 52.

Élimination, vol. $28, \mathrm{n}^{\circ} 2$, p. 162 .

Élimination d'atome, vol. 28, no $^{\circ}$, p. 168 .

Eliminatoire, vol. $28, \mathrm{n}^{\circ} 2$, p. 162

Elliptique, vol. 28, n $^{\circ} 2$, p. 162.

Embargo, vol. 28, no 4, p. 402.

Emissivité, vol. 28 , no 3 , p. 290 .

Emphase, vol. 28, no 1, p. 50.

Emploi de la langue française, vol. $28, n^{\circ} 1$, p. 91.

Emploi euphorique, vol. 28, $\mathrm{n}^{\circ} 2$, p. 208.

Emploi mêtaphorique, vol. $28, n^{\circ} 3$, p. 303.

Employé du service du contentieux, vol. $28, n^{\circ} 1$, p. 23.

Emprunt, vol. $28, \mathrm{n}^{\circ} 3$, p. 303.

En prévision de, vol. 28 , no 3, p. 269.

Enceinte acoustique, vol. $28, n^{\circ} 2$, p. 191

Enceinter, vol. $28, n^{\circ} 3$, p. 304.

Encodage, vol. 28, no 2 , p. 133.

Encyclopédisme, vol. 28, no 3, p. 305

Endommager, vol. 28, no 3, p. 304.

ène, vol. 28, no 2, p. 169.

Énergie renouvelable, vol. 28 , no 3 , p. 283.

Énergie solaire, vol. $28, n^{\circ} 3$, p. 283.

Enfourchement, vol. 28, no 4, p. 362.

Énoncé, vol. $28, n^{\circ} 2$, p. 142.

Enregistrement, vol. 28, no 2, p. 191.

Enseignement des langues, vol. 28, no 3, p. 310 .

Entrée, vol. 28, no 4, p. 366.

Entrée d'eau, vol. 28, n 3, p. 294

Entreprise de traduction, vol. 28, no 1 , p. 90

Environnement textuel immédiat, vol. $28, n^{\circ} 3$, p. 305.

Équarrissage des grumes, vol. 28, no 2, p. 195.

Equilibreur, vol. $28, n^{\circ} 4$, p. 402 .

Equilibreur avant-arrière, vol. 28 , no 4, p. 402.

Equivalence contextuelle, vol. $28, n^{\circ} 1$, p. 23.

Équivalence de situation, vol. 28, no $^{\circ} 1$, p. 23.

Equivalence fonctionnelle, vol. 28 , no 2 , p. 146.

erie, vol. $28, n^{\circ} 2$, p. 162. 
ESIT, vol. 28, no 1, p. 91.

Espace conceptuel, vol. $28, n^{\circ} 3$, p. 305

Espagnol, (traduction de 1'), vol. $28, n^{\circ} 1$, p. 48

Espéranto, vol. 28, n $^{\circ} 4$, p. 338.

Esperar, vol. 28, no $^{\circ}$, p. 37

Esprit, primauté de 1', vol. 28, n$^{\circ} 4$, p. 327.

Esquisse, vol. $28, n^{\circ} 4$, p. 402.

Étalonnage, vol. $28, n^{\circ} 4$, p. 366

Étalonné, vol. $28, n^{\circ} 4$, p. 366

Étape, vol. $28, \mathrm{n}^{\circ} 2$, p. 175

Ettat de langue, vol. $28, n^{\circ} 3$, p. 303

Éthanol ${ }^{2}$, vol. $28, n^{\circ} 2$, p. 178.

Éther diéthylique, vol. $28, n^{\circ} 2$, p. 168 .

Éther-oxyde, vol. 28, n $^{\circ} 2$, p. 168.

Éther-oxyde d'éthyle, vol. $28, n^{\circ} 2$, p. 168.

Ethnolinguistique, vol. 28, no $^{\circ}$, p. 48.

Étude, champ d', vol. 28, no 3, p. 280.

Étude terminologique et linguistique, vol. $28, n^{\circ} 4$, p. 358 .

Évaluation de la qualité en interprétation simultanée, vol. 28 , no 3, p. 236

Évaluation, feuille d', vol. 28, no $^{\circ}$, p. 243.

Évaluations, recueil des, vol. 28, no $^{\circ} 3$, p. 242

Évanouissement, vol. $28, n^{\circ} 4$, p. 402.

Exactitude atmosphérique, vol. 28 , no 1, p. 40.

Exclusion, critère d', vol. 28, n$^{\circ} 3$, p. 280.

Exercice comparatif, vol. $28, n^{\circ} 2$, p. 149

Exercice interprétatif, vol. $28, n^{\circ} 2$, p. 149.

Exercice traditionnel de la traduction, vol. 28 , $\mathrm{n}^{\circ} 3$, p. 312.

Exercices de morphosyntaxe, vol. 28, no 4, p. 412

Exhaustivité du recensement, vol. 28 , no 3 , p. 280 .

Expédier, vol. 28, no 2, p. 160.

Expéditeur, vol. 28, no 2, p. 160.

Expéditif, vol. 28 , n० 2, p. 160

Expédition, vol. 28, no 2, p. 160

Exploitation des données, vol. 28, no 3, p. 242.

Expression écrite, vol. $28, n^{\circ} 3$, p. 312.

Expression moyenâgeuse, vol. $28, n^{\circ} 1$, p. 40.

Expression orale, vol. $28, n^{\circ} 3$, p. 312

Expression polysémique, vol. 28, no $^{\circ}$, p. 208

Extension, vol. $28, n^{\circ} 2$, p. 208 ; vol. $28, n^{\circ} 3$, p. 303.

Extension maximale, vol. $28, n^{\circ} 2$, p. 208.

Extrusion, vol. $28, n^{\circ} 2$, p. 161.

Fable, vol. 28, no 1, p. 41

Fabrication du papier, vol. 28 , no 3 , p. 304

Facette du message verbal, vol. 28 , no 3 , p. 236.

Facteur de conversion, vol. 28 , no 3 , p. 290.

Facteur d'émission, vol. 28, n $^{\circ} 3$, p. 290.

Factorien, vol. $28, n^{\circ} 3$, p. 303 .

Faire acte de procédure, vol. $28, n^{\circ} 2$, p. 174

Faire cadeau, vol. 28, no 3, p. 303.

Fait, vol. $28, n^{\circ} 2$, p. 176.

Fait culturel, vol. $28, \mathrm{n}^{\circ} 2$, p. 148

Fait langagier, vol. $28, n^{\circ} 1$, p. 49

Fait linguistique, vol. $28, n^{\circ} 2$, p. 148 .

Fallecer, vol. 28 , no 1, p. 53.

Fannico, vol. 28 , no 3 , p. 303 .

Fantaliser, se, vol. 28, n $^{\circ} 3$, p. 304

Farde, vol. 28, no 3, p. 304 .

Fatigue, vol. $28, n^{\circ} 3$, p. 237.

Faute de langue, vol. $28, n^{\circ} 3$, p. 241.
Fauter, vol. $28, n^{\circ} 3$, p. 303 .

Faux ami, vol. 28 , no 3 , p. 267.

Fédération des coopératives, vol. $28, n^{\circ} 1$, p. 23.

Fermentation, vol. $28, n^{\circ} 2$, p. 179.

Fermer la bouche, vol. $28, n^{\circ} 3$, p. 303 .

Fermeture en fondu, vol. $28, n^{\circ} 4$, p. 402 .

Féticheur alaga, vol. $28, n^{\circ} 3$, p. 303.

Feuille d'évaluation, vol. $28, n^{\circ} 3$, p. 243.

Feuillet à tourner, vol. $28, n^{\circ} 4$, p. 362 .

Fibres du bois, à travers les, vol. 28 , n० 2 , p. 195.

Fibres du bois, dans le sens des, vol. 28 , no 2 , p. 195.

Fichier Recherche, vol. 28, no 2, p. 215.

Fichier Terminologie, vol. 28 , no 2 , p. 215.

Fichier Titres et appellations officielles, vol. 28, no 2, p. 215

Fidélité informationnelle, vol. $28, n^{\circ} 3$, p. 238 vol. 28, n$^{\circ} 3$, p. 239.

Figure de style, vol. $28, n^{\circ} 1$, p. 38.

Finition, sciage de, vol. 28, no $^{\circ} 2$, p. 196.

Fissuration, vol. $28, \mathrm{n}^{\circ} 3$, p. 293

Fixateur, vol. 28, n $^{\circ} 2$, p. 160.

Fixatif, vol. $28, n^{\circ} 2$, p. 160 .

Fixation, vol. $28, n^{\circ} 2$, p. 160

Fixer, vol. $28, n^{\circ} 2$, p. 160

Flèche, vol. 28, no 3 , p. 293.

Floresta, vol. 28, n$^{\circ} 1$, p. 37

Fon-fai, vol. $28, n^{\circ} 1$, p. 25.

Fonction, vol. 28 , no 2 , p. 167

Fonction compensatoire, vol. $28, n^{\circ} 2$, p. 208.

Fonction discriminante, vol. $28, n^{\circ} 2$, p. 208.

Fonction incrémentielle, vol. $28, n^{\circ} 2$, p. 208

Fonction, nom de, vol. 28 , no 2 , p. 168 ; vol. 28 , no 2, p. 169.

Fonctionnement, vol. $28, \mathrm{n}^{\circ} 2$, p. 173.

Fond de la vésicule biliaire, vol. 28, n $^{\circ} 4$, p. 388 .

Fond de l'estomac, vol. $28, n^{\circ} 4$, p. 388.

Fond de l'oeil, vol. 28 , no 4 , p. 387 .

Fond de l'utérus, vol. 28 , no 4 , p. 388.

Fond d'oeil, vol. 28 , no 4 , p. 387 .

Fondu, vol. $28, n^{0} 4$, p. 402 .

Fondu au noir, vol. 28, n $^{\circ} 4$, p. 402.

Fondu enchaîné, vol. 28 , no 4 , p. 402 .

Foquer, vol. $28, n^{\circ} 3$, p. 303 ; vol. 28 , no 3 , p. 304 .

Formalité, vol. 28 , no 2 , p. 175 .

Formation de mot, règle de, vol. $28, n^{\circ} 2$, p. 163 .

Formation de mots, règle de, vol. 28 , no 2 , p. 158 .

Formation de radicaux, règle de, vol. $28, n^{\circ} 2$, p. 157.

Forme, vol. $28, \mathrm{n}^{\circ} 2$, p. 142.

Forme, correspondance de, vol. $28, \mathrm{n}^{\circ} 2$, p. 133.

Forme déverbale, vol. 28 , no 2 , p. 165 .

Forme didactique, vol. $28, n^{\circ} 3$, p. 280.

Forme et sens, vol. $28, n^{\circ} 2$, p. 141.

Forme linguistique, vol. $28, n^{\circ} 2$, p. 138 .

Forme nominale, vol. 28 , no 1 , p. 50

Forme verbale, vol. 28 , no 1 , p. 50 .

Foutre le paquet, vol. $28, n^{\circ} 3$, p. 304.

Fraction, vol $28, n^{0} 2$, p. 162 .

Fracture, vol. 28, n०2, p. 162.

Français en Afrique noire, vol. 28, no 3, p. 302.

Français hors hexagone, vol. $28, n^{\circ} 3$, p. 303

Français universel, vol. $28, n^{\circ} 3$, p. 301.

Fréquence, vol. 28 , no 4 , p. 369.

Fréquence de résonance, vol. $28, n^{\circ} 2$, p. 191.

Fréquence, modulation de, vol. $28, \mathrm{n}^{\circ} 2$, p. 192. 
Friperie, vol. 28 , no 1 , p. 25 .

Fruta, vol. 28 , no 1 , p. 37 .

Fuels, classification des, vol. 28, no 3, p. 298.

Fulanis, vol. $28, \mathrm{n}^{\circ} 1$, p. 25.

Fusion, nom de, vol. 28, no 2 , p. 170.

Gain, vol. 28, no 2, p. 207.

Garcerie, vol. 28, n⿳ 3, p. 303.

Gari, vol. 28 , no 1 , p. 25

Garrot, vol. 28, no 2, p. 196.

$\mathrm{Gaz}$, vol. $28, \mathrm{n}^{\circ} 3$, p. 297.

Gaz, classification des, vol. 28, no 3, p. 298

Gaz de pétrole, liquéfaction des, vol. $28, n^{\circ} 3$, p. 297.

Gaz de remplacement, production de, vol. 28 , $\mathrm{n}^{\circ} 3$, p. 297.

Gaz naturel, liquéfaction du, vol. $28, \mathrm{n}^{\circ} 3$, p. 297.

Gazéification du charbon, vol. 28, no 3, p. 297

Gazette Provinciale du Québec, vol. 28, $\mathrm{n}^{\circ} 1$, p. 21.

Gazoduc géant, vol. 28, no 3 , p. 297.

Général, vol. $28, n^{\circ} 2$, p. 208.

Générateur mural, vol. 28, no 3, p. 299.

Génie automobile, vol. 28, no 4, p. 412.

Génie solaire, vol. $28, n^{\circ} 3$, p. 290.

Genièvre, vol. $28, n^{\circ} 2$, p. 185

Gente trabajando, vol. 28 , no 1 , p. 50.

Gentiane, vol. $28, \mathrm{n}^{\circ} 2$, p. 185 .

Gérondif, vol. $28, n^{\circ} 1$, p. 51

Gin, vol. $28, n^{\circ} 2$, p. 185 .

Goal-différence, vol. $28, \mathrm{n}^{\circ} 3$, p. 304.

Godard, Barbara, vol. 28 , no 4 , p. 352 .

Gombo, vol. $28, n^{\circ} 1$, p. 25.

Goujon, vol. 28, no 3 , p. 292

Goupille de fixation, vol. 28 , no 4 , p. 362

Goûter, vol. $28, n^{\circ} 3$, p. 303.

Gouttière de collecte, vol. $28, n^{\circ} 3$, p. 292.

Gouvernement canadien, banque de terminologie du, vol. $28, n^{\circ} 2$, p. 215.

Gouvernement canadien, réseau de terminologie du, vol. 28, n $^{\circ} 2$, p. 216 .

Grailler, vol. $28, n^{\circ} 3$, p. 304.

Grammaire normative, vol. $28, n^{\circ} 2$, p. 134.

Grammaire prescriptive, vol. $28, n^{\circ} 2$, p. 134

Grammatical, vol. $28, \mathrm{n}^{\circ} 2$, p. 140.

Grammaticalité, vol. $28, n^{\circ} 2$, p. 140.

Grand arrangeur, vol. $28, n^{\circ} 1$, p. 44

Grand pontife, vol. $28, n^{\circ} 1$, p. 44.

Grande circulation, vol. $28, n^{\circ} 4$, p. 384

Grande femme, vol. $28, n^{\circ} 3$, p. 303.

Grande scie de tonnelier, vol. $28, n^{\circ} 4$, p. 362

Grande scie de travers, vol. 28, no 2, p. 197 ; vol. $28, n^{\circ} 4$, p. 358 .

Grande zone partagée, vol. $28, \mathrm{n}^{\circ} 1$, p. 49.

Grandeur dentree secondaire, vol, $28,404, p .36$

Grandeur parasite, vol. $28, n^{\circ} 4$, p. 366.

Grandeur perturbatrice, vol. $28, n^{\mathrm{o}} 4$, p. 366

Grandeur physique, vol. 28 , no 4 , p. 369 .

Grandeur physique variable, vol. $28, \mathrm{n}^{\circ} 4$, p. 366.

Grandeur réglée, vol. $28, n^{\circ} 4$, p. 366 .

Graphique de circulation, vol. $28, n^{\circ} 3$, p. 305

Graphonomie, vol. $28, n^{\circ} 2$, p. 139.

Gras, vol. $28, n^{\circ} 2$, p. 184 .

Grave, vol. 28 , no 2 , p. 191 ; vol. 28 , no 4 , p. 385.
Grog, vol. $28, \mathrm{n}^{\circ} 2$, p. 185

Gros mot, vol. 28, no 3, p. 303.

Gros plan, vol. 28 , no 4 , p. 402

Groupe caractéristique, vol. $28, n^{\circ} 2$, p. 167.

Groupe de mots, vol. $28, n^{\circ} 2$, p. 133.

Groupe fonctionnel, vol. 28 , no 2, p. 167

Groupe principal, vol. $28, n^{\circ} 2$, p. 167.

Groupe secondaire, vol. 28, no 2, p. 167.

Groupement, vol. $28, n^{\circ} 2$, p. 167 .

Groupiste, vol. $28, n^{\circ} 4$, p. 402.

Grumes, équarrissage des, vol. $28, n^{\circ} 2$, p. 195.

Guindaille, vol. $28, \mathrm{n}^{\circ} 3$, p. 304

Haït, vol. $28, \mathrm{n}^{\circ} 2$, p. 162 .

Haïtien, vol. 28, no 2, p. 162.

Halogène, vol. 28 , no 2 , p. 166 ; vol. $28, \mathrm{n}^{\circ} 2$, p. 167

HANSARD, vol. $28, n^{\circ} 1$, p. 21.

Harmonique, vol. $28, n^{\circ} 2$, p. 191

Haut-parleur, vol. $28, n^{\circ} 2$, p. 191.

Haute fidélité, vol. $28, \mathrm{n}^{\circ} 2$, p. 191

Héliotechnique, vol. $28, n^{\circ} 3$, p. 290.

Hen-, vol. $28, n^{\circ} 2$, p. 170

Hendéca-, vol. $28, \mathrm{n}^{\mathrm{o}} 2$, p. 170

Hertz, vol. $28, \mathrm{n}^{\circ} 2$, p. 191.

Hétéro-atome, vol. $28, \mathrm{n}^{\circ} 2$, p. 167.

Hétéro-atomes, vol. $28, n^{\circ} 2$, p. 166.

Hétérocycle condensé, vol. $28, n^{\circ} 2$, p. 170.

Hétérogénéité de surface, vol. $28, \mathrm{n}^{\circ} 1$, p. 51 .

Hexane, vol. $28, n^{\circ} 2$, p. 166 ; vol. $28, n^{\circ} 2$, p. 170

Hi-fi, vol. $28, n^{\circ} 2$, p. 191

Hifi, vol. $28, n^{\circ} 2$, p. 191.

Homogénéité dans le repérage, vol. $28, n^{\circ} 3$, p. 280.

Hors jeu, vol. $28, \mathrm{n}^{\circ} 3$, p. 304

Hydro-, vol. 28, no $^{\circ}$, p. 168.

Hydrocarbure acyclique, vol. $28, n^{\circ} 2$, p. 169.

Hydrocarbure cyclique, vol. $28, \mathrm{n}^{\circ} 2$, p. 169.

Hydrogène, vol. $28, n^{\circ} 2$, p. 166.

Hydromel, vol. $28, n^{\circ} 2$, p. 184.

Hydroxy-5 pentanone- 2 , vol. $28, n^{\circ} 2$, p. 167.

Hydroxy-, vol. $28, n^{\circ} 2$, p. 167.

Hydroxypentanone, vol. $28, n^{\circ} 2$, p. 167.

Hyperpressé, vol. $28, n^{\circ} 4$, p. 378 .

Hyperpresseur, vol. $28, n^{\circ} 4$, p. 378

Hyperpression, vol. 28, no $^{\mathrm{o}} 4$, p. 378

Hypertendu, vol 28 , no 4 , p. 378 .

Hypertensif, vol. $28, n^{\circ} 4$, p. 378.

Hypertension, vol. $28, n^{\circ} 4$, p. 378 .

Hypertension artérielle, vol. $28, n^{\circ} 4$, p. 376

Hypertension bénigne, vol. $28, n^{\circ} 4$, p. 385 .

Hypertension maligne, vol. $28, n^{\circ} 4$, p. 385 .

Hypocras à la framboise, vol. $28, \mathrm{n}^{\circ} 2$, p. 185.

Hyponymie, vol. $28, n^{\circ} 4$, p. 393.

$\mathrm{Hz}$, vol. $28, \mathrm{n}^{\circ} 2$, p. 191

IC, vol. $28, \mathrm{n}^{\circ} 2$, p. 151

Idéologie translatologique, vol. $28, \mathrm{n}^{\circ} 2$, p. 208.

Illustration, vol. $28, n^{\circ} 3$, p. 281

Images d'archives, vol. $28, n^{\circ} 4$, p. 402.

Imparfait, vol. $28, n^{\circ} 3$, p. 313 .

Impératif, vol. $28, n^{\circ} 1$, p. 52 . 
Implication méthodologique, vol. $28, \mathrm{n}^{\mathrm{0}} 4$, p. 330 . Impôt sur le bétail, vol. $28, \mathrm{n}^{\circ} 1$, p. 26. Imprégnation alcoolique, vol. 28, $\mathrm{n}^{\circ} 2, \mathrm{p} .184$. Improductif, vol. $28, n^{\circ} 3$, p. 257. Incident, vol. $28, \mathrm{n}^{\circ} 2$, p. 176 Incident d'interprétation, vol. $28, n^{\circ} 3$, p. 241. Inclinaison, vol. $28, n^{\circ} 3$, p. 290. Incompris sophistiqué, vol. $28, \mathrm{n}^{\circ} 2$, p. 207 Incompris trivialisé, vol. $28, \mathrm{n}^{\circ} 2$, p. 207. Index des profils, vol. $28, \mathrm{n}^{\circ} 2$, p. 218. Indice de position, vol. $28, \mathrm{n}^{\circ} 2$, p. 167 Indigènement, vol. $28, \mathrm{n}^{\circ} 3$, p. 303.

Industrie du gaz, vol. 28, no $^{\circ}$, p. 297 Industrie papetière, vol. $28, \mathrm{n}^{\circ} 3$, p. 304.

Infécond, vol. $28, \mathrm{n}^{\circ} 3$, p. 257.

Infidélité, vol. $28, n^{\circ} 3$, p. 241 .

Infiltration, vol. $28, \mathrm{n}^{\circ} 3$, p. 293

Inflation, vol. $28, n^{\circ} 2$, p. 161.

Influence de l'audio-visuel, vol. 28, no $^{\circ}$, p. 312.

Information, vol. $28, \mathrm{n}^{\circ} 2$, p. 218

Inhibiteur, vol. $28, n^{\circ} 4$, p. 388.

Insaturation, vol. $28, n^{\circ} 2$, p. 168 .

Insertion lexicale, vol. $28, n^{\circ} 2$, p. 159.

Insolateur à chauffage d'air, vol. $28, n^{\circ} 3$, p. 290.

Insolateur à fonction thermique, vol. $28, n^{\circ} 3$, p. 290.

Insolateur à lame d'air, vol. $28, n^{\circ} 3$, p. 290. Insolateur concentrateur, vol. $28, n^{\circ} 3$, p. 290.

Insolateur plan, vol. $28, \mathrm{n}^{\circ} 3$, p. 290.

Instruction, acte d', vol. 28 , no 2 , p. 174 ; vol. 28 , $\mathrm{n}^{\circ} 2$, p. 175

Instrument de diffusion des idées, vol. $28, \mathrm{n}^{\circ} 4$, p. 343 .

Instrument d'organisation, vol. $28, n^{\circ} 3$, p. 280.

Intégralité du message, vol. $28, n^{\circ} 4$, p. 323.

Intégration, critère d', vol. $28, \mathrm{n}^{\circ} 3$, p. 303 .

Interférence, vol. $28, n^{\circ} 3$, p. 303.

Interférence linguistique, vol. $28, \mathrm{n}^{\circ} 1$, p. 24.

Interjection, vol. $28, n^{\circ} 3$, p. 299.

Internégatif, vol. $28, n^{\circ} 4$, p. 402 .

Interprétation, vol. $28, \mathrm{n}^{\circ} 1$, p. 94 ; vol. $28, \mathrm{n}^{\circ} 2$, p. 133.

Interprétation auprès des tribunaux, vol. $28, \mathrm{n}^{\circ} 2$, p. 213.

Interprétation consécutive, vol. $28, \mathrm{n}^{\circ} 3$, p. 242.

Interprétation, incident $d^{\prime}$, vol. $28, \mathrm{n}^{\circ} 3, \mathrm{p} .241$.

Interprétation, qualité de $\mathrm{r}^{\prime}$, vol. $28, \mathrm{n}^{\mathrm{0}} 3$, p. 239.

Interprétation simultanée, vol. $28, \mathrm{n}^{\circ} 3$, p. 236 .

Interprète actif, vol. $28, n^{\circ} 3$, p. 240.

Interprète judiciaire, vol. $28, \mathrm{n}^{\mathrm{o}} 2$, p. 213.

Interprète "occasionnel ", vol. $28, \mathrm{n}^{\mathrm{0}} 1$, p. 18.

Interprète, rôle de l', vol. $28, \mathrm{n}^{\circ} 1$, p. 94.

Intraduisible, vol. $28, \mathrm{n}^{\circ} 1$, p. 44.

Intrusion, vol. $28, \mathrm{n}^{\circ} 2$, p. 161.

Intuition linguistique, vol. $28, \mathrm{n}^{\circ} 2$, p. 134

Inventaire des particularités lexicales du français

en Afrique noire, vol. $28, n^{\circ} 3$, p. 302

-ion, vol. $28, \mathrm{n}^{\circ} 2$, p. 158.

Ir, vol. $28, n^{\circ} 1$, p. 37

Isolation variable, vol. $28, n^{\circ} 3$, p. 290.

Isotopie, vol. $28, \mathrm{n}^{\circ} 2, \mathrm{p} .207$.

Itinéraire orateur-interprète-délégués, vol. $28, \mathrm{n}^{\circ} 3$, p. 238.

p. 238.
-ition, vol. $28, n^{\circ} 2$, p. 158.
Jadis, vol. $28, \mathrm{n}^{\circ} 1$, p. 37

Jangali tax, vol. $28, \mathrm{n}^{\circ} 1$, p. 26.

Jeu, vol. $28, n^{\circ} 2$, p. 207.

JEU, modèle du, vol. $28, n^{\circ} 2$, p. 207.

Jointement, vol, $28, n^{\circ} 3$, p. 293.

Joue de tenon, vol. $28, \mathrm{n}^{\circ} 2$, p. 196

Journal officiel, vol. $28, \mathrm{n}^{\circ} 1$, p. 21.

Journalisme électronique, vol. $28, n^{\circ} 4$, p. 402.

Juguler l'hypertension, vol. $28, n^{\circ} 4$, p. 386 .

Justice, action en, vol. $28, n^{\circ} 2$, p. 173.

Justice, poursuite en, vol. $28, \mathrm{n}^{\circ} 2$, p. 173.

$\mathrm{KHz}$, vol. $28, \mathrm{n}^{\circ} 2$, p. 191.

Kilohertz, vol. 28, no 2, p. 191.

Kirsh, vol. 28, nº 2, p. 185.

LA, vol. $28, \mathrm{n}^{\circ} 2$, p. 131

Lame, vol. 28, n $^{\circ} 2$, p. 193 ; vol. $28, n^{\circ} 2$, p. 196.

Lame d'air, vol. $28, n^{\circ} 3$, p. 292

Lame fixe, vol. $28, n^{\circ} 2$, p. 192 ; vol. $28, n^{\circ} 4$, p. 358.

Lame large, vol. $28, \mathrm{n}^{\circ} 2$, p. 194.

Lame orientable, vol. $28, n^{\circ} 2$, p. 192 ; vol. 28 , $n^{\circ} 4$, p. 361 .

Langage, vol. $28, \mathrm{n}^{\circ} 2$, p. 141 .

Langage de la régulation, vol. $28, \mathrm{n}^{\mathrm{o}} 4$, p. 365 .

Langage papetier, vol. $28, n^{\circ} 3$, p. 305 .

Langue authentique, vol. $28, n^{\circ} 3$, p. 313

Langue cible, vol. $28, \mathrm{n}^{\circ} 2$, p. 131.

Langue coloniale, vol. $28, \mathrm{n}^{\circ} 1$, p. 17.

Langue-culture, vol. $28, n^{\circ} 1$, p. 44

Langue d'arrivée, vol. $28, \mathrm{n}^{\circ} 2$, p. 131.

Langue de départ, vol. $28, \mathrm{n}^{\circ} 2$, p. 131.

Langue de l'original, vol. $28, \mathrm{n}^{\circ} 2, \mathrm{p} .131$

Langue de traduction, vol. $28, \mathrm{n}^{\circ} 2, \mathrm{p} .131$.

Langue écrite, vol. 28, $\mathrm{n}^{\circ} 2$, p. 144.

Langue écrite soutenue, vol. $28, n^{\circ} 3$, p. 313.

Langue importée, vol. $28, \mathrm{n}^{\mathrm{0}} 1$, p. 17.

Langue indigène, vol. $28, n^{\circ} 1$, p. 17.

Langue locale, vol. $28, n^{\circ} 1$, p. 17

Langue, moyen d'enregistrer, vol. $28, n^{\circ} 2$, p. 138.

Langue parlée, vol. $28, n^{\circ} 2$, p. 144 ; vol. $28, n^{\circ} 3$, p. 313 .

Langue-parole, vol. $28, \mathrm{n}^{\circ} 2$, p. 142.

Langue répertoire, vol. $28, \mathrm{n}^{\circ} 1$, p. 49

Langue source, vol. $28, \mathrm{n}^{\circ} 2$, p. 131.

Langue-système, vol $28, n^{0} 1$, p. 44.

Langue, traduction de, vol. $28, n^{\circ} 2$, p. 149.

Langue vernaculaire, vol. $28, \mathrm{n}^{\circ} 1, \mathrm{p} .17$.

Langue vivante, vol. $28, \mathrm{n}^{\circ} 3$, p. 299.

Lanrido, vol. $28, n^{\circ} 1$, p. 25.

Larmier, vol. $28, n^{\circ} 3$, p. 293.

LC, vol. $28, n^{\circ} 2$, p. 131

LD, vol. $28, n^{\circ} 2$, p. 131 .

Lecteur, vol. $28, \mathrm{n}^{\mathrm{0}} 2$, p. 160.

Lecteur, traducteur-, vol. $28, n^{0} 2$, p. 207.

Lecture, vol. $28, n^{\circ} 3$, p. 237.

Lecture du texte, vol. $28, n^{\circ} 2$, p. 207.

Lecture, table de, vol. 28, no $^{\circ}$, p. 192.

Légère, vol. $28, n^{\circ} 4$, p. 385 .

Lettre officielle, vol. $28, n^{\circ} 1$, p. 21

Lettre, respect de la, vol. $28, n^{\circ} 4$, p. 326. 
Lexicographie générale, vol. 28, no 3, p. 279.

Lexicographie structurale, vol. $28, n^{\circ} 3$, p. 305

Lexicographie terminologique, vol. $28, \mathrm{n}^{\circ} 3$ p. 279.

Lexie, vol. 28 , no 1 , p. 54.

Lexie complexe, vol. $28, \mathrm{n}^{\circ} 3$, p. 282.

Lexique de l'audiophonie, vol. 28, n०2, p. 191.

Lexique du chauffage solaire, vol. 28, n $^{\circ} 3$, p. 288

Liant, vol. $28, n^{\circ} 1$, p. 93 .

Lien, vol. 28, no $^{\circ}$, p. 93 .

Lien interchamp, vol. $28, n^{\circ} 1$, p. 54.

Lien intratextuel, vol. 28, no $^{\circ}$, p. 207

Lien sémantique, vol. 28 , no 1, p. 53.

Linguiste, vol. $28, n^{\circ} 3$, p. 279.

Linguiste praticien, vol. $28, \mathrm{n}^{\circ} 2$, p. 134

Linguistique appliquée, vol, 28, no 2, p. 134.

Linguistique contemporaine, vol. $28, \mathrm{n}^{\circ} 2$, p. 144

Linguistique et traduction, vol. $28, n^{\circ} 2$, p. 130

Linguistique formelle, vol. $28, n^{\circ} 2$, p. 140.

Linguistique, maturité de la, vol. $28, \mathrm{n}^{\circ} 2$, p. 151 .

Linguistique structurale, vol. $28, \mathrm{n}^{\circ} 2$, p. 144.

Linguistique traditionnelle, vol. $28, \mathrm{n}^{\circ} 2$, p. 144.

Linguitrad, vol. $28, n^{\circ} 4$, p. 324.

Liquéfaction des gaz de pétrole, vol. $28, n^{\circ} 3$, p. 297.

Liquéfaction du gaz naturel, vol. 28, no 3, p. 297

Liqueur alcoolique, vol. $28, \mathrm{n}^{\circ} 2$, p. 179

Liqueur douce, vol. $28, \mathrm{n}^{\circ} 2$, p. 185.

Liqueur sèche, vol. 28, n $^{\circ} 2$, p. 184.

Liqueur, vin de, vol. $28, n^{\circ} 2$, p. 184.

Litige, vol. 28, $n^{\circ} 2$, p. 174

LO, vol. $28, \mathrm{n}^{\circ} 2$, p. 131.

Localisation par l'ouie, vol. $28, \mathrm{n}^{\circ} 2$, p. 192

Loi, vol. 28 , no 4 , p. 370 ; vol. 28 , no 4 , p. 370

Loi de consigne, yol. 28 , no 4 , p. 366 .

Loi sur les crédits budgétaires, vol. $28, n^{\circ} 4$, p. 370.

Longueur des phrases, vol. $28, \mathrm{n}^{\circ} 1$, p. 37.

LS, vol. $28, \mathrm{n}^{\circ} 2$, p. 131

LT, vol. $28, n^{\circ} 2$, p. 131

Lua, vol. 28, no $^{\circ}$, p. 37.

Maçonnerie en briques, vol. 28, n० 3, p. 292.

Macro-contexte, vol. 28, no 3, p. 305.

Macro-discipline, vol. $28, n^{\circ} 3$, p. 280

Madère, vol. 28 , no 2 , p. 184

Madrier, vol. 28, no 2, p. 196.

Magnétophone, platine de, vol. 28, n², p. 192.

Maillet, Antonine, vol. $28, n^{\circ} 4$, p. 352.

Maintenir la tension, vol. $28, n^{\circ} 4$, p. 364 .

Maîtriser l'hypertension, vol. 28, no 4 , p. 386.

Maladresse, vol. $28, n^{\circ} 3$, p. 241.

Malaga, vol. $28, n^{\circ} 2$, p. 184

Maligne, vol. 28 , no 4 , p. 385 .

Mandat, vol. $28, n^{\circ} 4$, p. 370 .

Maquette, vol. $28, n^{\circ} 4$, p. 402.

Martini, vol. $28, n^{\circ} 2$, p. 184.

Martyr de la traduction, vol. $28, n^{\circ} 4$, p. 341.

Matabiche, vol. $28, \mathrm{n}^{\circ} 3$, p. 303 .

Matériel audiophonique, vol. 28, no 2, p. 192

Matière, problème de, vol. $28, n^{\circ} 2$, p. 134.

Maturité de la linguistique, vol. 28 , no 2 , p. 151.

Mécanique, traduction, vol. $28, n^{\circ} 2$, p. 137.

Média, vol. 28, no 4, p. 402.
Médialogie, vol. 28, no 4, p. 402.

Médialogue, vol. $28, \mathrm{n}^{\circ} 4$, p. 402 .

Médium graphique, vol. $28, \mathrm{n}^{\circ} 2$, p. 144

Médium phonique, vol. $28, \mathrm{n}^{\circ} 2$, p. 144.

Mélange eutectique, vol. $28, n^{\circ} 3$, p. 290

Membre d'une commission, vol. 28 , no 4 , p. 372

Mémoire, vol. 28, no 2, p. 205.

Mémoire immédiate, vol. $28, \mathrm{n}^{\circ} 2$, p. 205.

Meneau, vol. 28, no $^{\circ}$, p. 293.

Mensonge, vol. $28, n^{\circ} 2$, p. 162.

Menthe, vol. 28, n $^{\circ} 2$, p. 185.

Mentir, vol. $28, n^{\circ} 2$, p. 162 .

Message écrit, vol. 28, no 3, p. 237.

Message, intégralité du, vol. $28, n^{\circ} 4$, p. 323

Message, transmission du, vol. $28, n^{\circ} 3$, p. 237

Message verbal, vol. $28, \mathrm{n}^{\circ} 3$, p. 236.

Mesure, vol. $28, \mathrm{n}^{\circ} 2$, p. 175 .

Métaphore, vol. $28, n^{\circ} 2$, p. 207

Méthane, vol. 28, no 2, p. 166

Méthanier, vol. 28, no 3, p. 297.

Méthanol, vol. $28, n^{\circ} 2$, p. 168 .

Méthode de nomenclature, vol. $28, n^{\circ} 2$, p. 167.

Méthyl-éthyl-cétone, vol. $28, \mathrm{n}^{\circ} 2$, p. 168.

Microchamp, vol. $28, \mathrm{n}^{\circ} 1$, p. 54.

Microsystème sémantique, vol. $28, \mathrm{n}^{\circ} 3$, p. 305.

Milieu extérieur, vol. 28 , no 4 , p. 366.

Mini-disque, vol. 28, no 4 , p. 403

Minimum sémique, vol. $28, n^{\circ} 1$, p. 52 .

Mirabelle, vol. 28 , no 2, p. 185.

Mise en forme, vol. $28, \mathrm{n}^{\circ} 2$, p. 133.

Mise en phase, vol. $28, n^{\circ} 2$, p. 192

Mode de création, vol. 28, n $^{\circ} 3$, p. 299.

Modèle du JEU, vol. 28 , n० 2, p. 207.

Modérée, vol. 28, n० 4, p. 385.

Modulation d'amplitude, vol. 28, n०2, p. 192.

Modulation de fréquence, vol. $28, \mathrm{n}^{\circ} 2$, p. 192

Molécule, vol. 28, n², p. 167.

Monde ethnographique, vol. $28, n^{\circ} 4$, p. 332.

Monde littéraire canadien, vol. 28 , no 4 , p. 352 .

Monde, vision du, vol. $28, n^{\circ} 4$, p. 330

Moniteur, vol. 28, no 4 , p. 403.

Montage sur copie, vol. $28, n^{\circ} 4$, p. 403.

Montage sur original, vol. $28, n^{\circ} 4$, p. 403 .

Montagnais, vol. $28, \mathrm{n}^{\circ} 2$, p. 162 .

Montant, vol. $28, n^{\circ} 3$, p. 293.

Montée, temps de, vol. 28, $\mathrm{n}^{\circ} 2$, p. 192

Monter sec, vol. $28, n^{\circ} 4$, p. 403.

Monter serré, vol. $28, n^{\circ} 4$, p. 403.

Monture, vol. $28, \mathrm{n}^{\circ} 2$, p. 196 ; vol. $28, \mathrm{n}^{\circ} 2$, p. 197.

Monture de bois, vol. $28, n^{\circ} 2$, p. 192.

Monture tubulaire, vol. $28, \mathrm{n}^{\circ} 2$, p. 197 ; vol. 28 , $\mathrm{n}^{\circ} 4$, p. 363.

Morar, vol. 28, no 1, p. 37.

Morir, vol. $28, n^{\circ} 1$, p. 53

Morphème lexical, vol. 28 , no 1, p. 53

Morphème lexical générateur, vol. 28, n 3, p. 299.

Morphème lié, vol. $28, \mathrm{n}^{\circ} 2$, p. 158

Mort, vol. $28, n^{\circ} 1$, p. 53.

Mortaise à mi-bois, vol. $28, n^{\circ} 2$, p. 193

Mot, problématique du, vol. 28, no 3, p. 299.

Motivation, vol. $28, n^{\circ} 1$, p. 55 ; vol. $28, n^{\circ} 4$, p. 334.

Mourir, vol. 28, n 1, p. 53.

Mouvement néologique, vol. 28, no 3, p. 299.

Moyen de droit, vol. 28 , no 2, p. 175 
Moyen d'enregistrer la langue, vol. $28, \mathrm{n}^{\circ} 2$, p. 138.

Moyen terme, à, vol. 28, no 2, p. 205.

Moyenne zone partagée, vol. $28, \mathrm{n}^{\circ} 1$, p. 49

Mur à double paroi, vol. 28, no $^{\circ}$, p. 292.

Mur capteur, vol. $28, n^{\circ} 3$, p. 290.

Mur d'eau, vol. 28 , n 3, p. 290.

Mur en maçonnerie, vol. $28, n^{\circ} 3$, p. 293.

Mur extérieur, vol. 28, no 3, p. 292.

Mur-rideau, vol. $28, n^{\circ} 3$, p. 293.

Mur Trombe, vol. 28, no 3, p. 290.

Mur Trombe-Michel, vol. $28, n^{\circ} 3$, p. 290

Murmure, vol. $28, n^{\circ} 4$, p. 381 .

Murmure vésiculaire, vol. $28, n^{\circ} 4$, p. 381 .

Musique, vol. 28, no 1 , p. 40

Mystification, vol. $28, n^{\circ} 4$, p. 334 .

Mythe de l'intraduisible, vol. 28 , no 1, p. 44

Naranja, vol. $28, n^{\circ} 1$, p. 51.

Nature des oxydants, vol. $28, n^{\circ} 3$, p. 305

Néo-firthiens, vol. $28, \mathrm{n}^{\circ} 2$, p. 137.

Néologie, vol. $28, \mathrm{n}^{\circ} 3$, p. 282 ; vol. $28, \mathrm{n}^{\circ} 3$, p. 299.

Néologie de langue, vol. $28, n^{\circ} 3$, p. 299.

Néologie dénominative, vol. $28, \mathrm{n}^{\circ} 3$, p. 299.

Néologie par emprunt, vol. 28, no 3, p. 299.

Néologie phonologique, vol. $28, n^{\circ} 3$, p. 299.

Néologie sémantique, vol. $28, n^{\circ} 3$, p. 299.

Néologie syntagmatique, vol. $28, \mathrm{n}^{\circ} 3$, p. 299.

Néologisme français, vol. $28, n^{\circ} 3$, p. 299.

Nid d'abeilles, vol. $28, n^{\circ} 3$, p. 290 .

Nitro-II tétraoxa-2,5,8,10 undécana, vol. $28, n^{\circ} 2$ p. 169.

Niveau de langue, vol. $28, n^{\circ} 1$, p. 24 ; vol. 28 , $n^{\circ} 3$, p. 303.

Noeud, vol. $28, \mathrm{n}^{\circ} 1$, p. 54.

Noeud atrio-ventriculaire, vol. $28, n^{\circ} 4$, p. 381.

Nom additif fonctionnel, vol. $28, \mathrm{n}^{\circ} 2$, p. 168.

Nom de fonction, vol. $28, \mathrm{n}^{\circ} 2$, p. 168 ; vol. 28 , $\mathrm{n}^{\circ} 2$, p. 169.

Nom de fusion, vol. $28, n^{\circ} 2$, p. 170.

Nom fondamental, vol. $28, \mathrm{n}^{\circ} 2$, p. 166 .

Nom radico-fonctionnel, vol. $28, \mathrm{n}^{\mathrm{o}} 2$, p. 168.

Nom semi-trivial, vol. $28, n^{\circ} 2$, p. 166 .

Nom soustractif, vol. $28, \mathrm{n}^{\circ} 2$, p. 168.

Nom substitutif, vol. $28, n^{\circ} 2$, p. 167.

Nom systématique, vol. $28, \mathrm{n}^{\circ} 2$, p. 166.

Nom trivial, vol. $28, n^{\circ} 2$, p. 166 .

Nombre de dents, vol. $28, \mathrm{n}^{\mathrm{0}} 2$, p. 196

Nombre indexé, vol. $28, n^{\circ} 2$, p. 171 .

Nomenclature additive, vol. $28, \mathrm{n}^{\circ} 2$, p. 168

Nomenclature conjonctive, vol. $28, n^{\circ} 2$, p. 169

Nomenclature des composés organiques, vol. 28 , $\mathrm{n}^{\circ} 2$, p. 166.

Nomenclature en " a ", vol. $28, n^{\circ} 2$, p. 169.

Nomenclature, méthode de, vol. $28, \mathrm{n}^{\circ} 2$, p. 167.

Nomenclature par remplacement, vol. $28, \mathrm{n}^{\circ} 2$, p. 169.

Nomenclature radico-fonctionnelle, vol. $28, n^{\circ} 2$, p. 168.

Nomenclature soustractive, vol. $28, \mathrm{n}^{\circ} 2$, p. 168 .

Nomenclature substitutive, vol. $28, \mathrm{n}^{\circ} 2$, p. 167.

Non-respect des niveaux de langue, vol. $28, n^{\circ} 1$, p. 24.
Nor-, vol. $28, \mathrm{n}^{\circ} 2$, p. 169.

Norpinane, vol. $28, n^{\circ} 2$, p. 169.

Note de bas de page, vol. $28, n^{\circ} 3$, p. 305 .

Notes de terminologie, vol. $28, \mathrm{n}^{\circ} 1$, p. 22

Notes de traduction, vol. $28, \mathrm{n}^{\circ} 1$, p. 22

Notion, vol. 28, n० 3, p. 283.

Notions, présentation des, vol. $28, n^{\circ} 3$, p. 280 .

Notions, regroupement des, vol. $28, \mathrm{n}^{\circ} 3$, p. 280.

Nouvelle-Calédonie, vol. $28, \mathrm{n}^{\circ} 1$, p. 17.

Nouvelle-Guinée, vol. $28, \mathrm{n}^{\circ} 1$, p. 18

Noyaux sémique, vol. $28, \mathrm{n}^{\circ} 1$, p. 52.

Numérotage, vol. $28, n^{\circ} 2$, p. 171 .

Objet, vol. $28, \mathrm{n}^{\circ} 2$, p. 148.

Océanie, vol. $28, \mathrm{n}^{\circ} 1$, p. 17

Océanie occidentale, vol. $28, n^{\circ} 1$, p. 17.

Océanie septentrionale, vol. $28, \mathrm{n}^{\circ} 1$, p. 17.

OIT, vol. 28, no $^{\circ}$, p. 19.

Okirika, vol. $28, \mathrm{n}^{\circ} 1$, p. 25.

Okro, vol. 28 , no 1, p. 25.

-olactone, vol. $28, \mathrm{n}^{\circ} 2$, p. 169

-olide, vol. $28, \mathrm{n}^{\circ} 2$, p. 169.

Onde acoustique, vol. $28, \mathrm{n}^{\circ} 2$, p. 192.

Onde sonore, vol. $28, \mathrm{n}^{\circ} 2$, p. 192

Onde sphygmique, vol. $28, \mathrm{n}^{\circ} 4$, p. 377.

Ondulation, vol. $28, \mathrm{n}^{\circ} 2$, p. 162 .

Ondulatoire, vol. $28, \mathrm{n}^{\circ} 2$, p. 162.

-one, vol. $28, \mathrm{n}^{\circ} 2$, p. 167.

Onomasiologie, vol. $28, \mathrm{n}^{\circ} 2$, p. 133

Onomatopée, vol. $28, n^{\circ} 3$, p. 299.

Opérateur de prises de vues, vol. $28, n^{\circ} 4$, p. 403

Opération, vol. $28, \mathrm{n}^{\circ} 2$, p. 174 ; vol. $28, \mathrm{n}^{\circ} 2$, p. 175.

Opération de transfert, vol. $28, \mathrm{n}^{\circ} 4$, p. 324

Opération juridique, vol. $28, \mathrm{n}^{\circ} 2$, p. 175 .

Opération traductionnelle, vol. $28, \mathrm{n}^{\circ} 2$, p. 131.

Opération traduisante, vol. $28, n^{\circ} 4$, p. 323 .

Option didactique, vol. 28, no $^{\circ}$, p. 280.

Option formelle, vol. $28, \mathrm{n}^{\circ} 3$, p. 280.

-or, vol. $28, \mathrm{n}^{\circ} 2$, p. 157 .

Orange, vol. $28, \mathrm{n}^{\circ} 1$, p. 51 .

Ordonnance de cassation, vol. $28, n^{\circ} 4$, p. 371.

Ordonnateur, vol. $28, n^{\circ} 2$, p. 160.

Ordre alphabétique, vol. 28 , no 3 , p. 280 ; vol. 28 , $n^{\circ} 3$, p. 282.

Oreillette, vol. $28, n^{\circ} 4$, p. 381.

Organe de réglage, vol. $28, n^{\circ} 4$, p. 367.

Organisation de la documentation, vol. $28, \mathrm{n}^{\circ} 2$, p. 217.

Organisation, instrument d', vol. $28, n^{\circ} 3$, p. 280 .

Organisation, sigle d', vol. $28, \mathrm{n}^{\circ} 3$, p. 298.

Orientation auditive, vol. $28, n^{\circ} 2$, p. 192.

Outil d'assemblage, vol. $28, n^{\circ} 4$, p. 359.

Outil manuel de sciage, vol. $28, \mathrm{n}^{\circ} 2$, p. 192.

Ouverture en fondu, vol. $28, n^{\circ} 4$, p. 403.

Ouvir, vol. $28, \mathrm{n}^{\circ} 1$, p. 37.

Oxa-, vol. $28, n^{\circ} 2$, p. 169.

Oxyde d'éthyle, vol. $28, \mathrm{n}^{\circ} 2$, p. 168.

Oxyde d'éthylène, vol. $28, \mathrm{n}^{\circ} 2$, p. 168.

Oxyde nitrique, vol. $28, \mathrm{n}^{\circ} 3$, p. 298.

Oxygène, vol. $28, \mathrm{n}^{\circ} 2$, p. 166. 
Palacio, vol. 28, no 1, p. 37.

Pancarte, vol. $28, n^{\circ} 1$, p. 50

Papier, vol. 28, no 3 , p. 304

Papier - Procédés et matériel, le, vol. 28, no 3 , p. 304.

Parasynonyme, vol. $28, n^{\circ} 1$, p. 53 ; vol. $28, n^{\circ} 1$, p. 54.

Pare-vapeur, vol. 28, no $^{\circ} 3$, p. 294.

Parlementaire, vol. $28, n^{\circ} 1$, p. 23

Paroi, vol. 28, no $^{\circ}$, p. 294.

Parole-langue, vol. $28, n^{\circ} 2$, p. 142.

Parrainage, vol. $28, n^{\circ} 4$, p. 403

Parrainer, vol. $28, n^{\circ} 4$, p. 403

Particularisation, vol. 28, no $^{\circ}$, p. 303

Particularité, vol. $28, \mathrm{n}^{\circ} 3$, p. 303

Particulier, vol. $28, \mathrm{n}^{\circ} 2$, p. 208

Partie, vol. 28, $\mathrm{n}^{\circ}$ 2, p. 162.

Partiel, vol. 28, $\mathrm{n}^{\circ} 2$, p. 162

Pas, vol. 28, no 2, p. 195

Passage, donner du, vol. $28, \mathrm{n}^{\circ} 2$, p. 194.

Passation de service, rapport de, vol. $28, n^{\circ} 1$, p. 23.

Passé composé, vol. $28, n^{\circ} 3$, p. 313

Passé défini, vol. $28, n^{\circ} 3$, p. 313 .

Passé simple, vol. $28, \mathrm{n}^{\circ} 1$, p. 38 ; vol. $28, \mathrm{n}^{\circ} 3$, p. 313.

Pastis, vol. 28, no 2, p. 185.

Pastis 51, vol. 28, no 2, p. 185

Pâte à papier, vol. $28, n^{\circ} 3$, p. 305 .

Pathos théoriciste, vol. $28, \mathrm{n}^{\circ} 2$, p. 132

Patrimoine culturel français, vol. $28, n^{\circ} 1$, p. 41

Pays mélanésien, vol. 28, no 1, p. 17.

Pays micronésien, vol. $28, n^{\circ} 1$, p. 17

Pays océanien, vol. $28, n^{\circ} 1$, p. 18

Pays polynésien, vol. $28, n^{\circ} 1$, p. 17

Pédagogie de la traduction, vol. $28, n^{\circ} 1$, p. 94 ; vol. $28, n^{\circ} 2$, p. 206.

Pédagotrad, vol. $28, n^{\circ} 4$, p. 324 ; vol. $28, n^{\circ} 4$, p. 331.

Pététration de l'eau de pluie, vol. $28, n^{\circ} 3$, p. 294

Pentachloro-, vol. $28, \mathrm{n}^{\circ} 2$, p. 170

Pentadiène, vol. $28, n^{\circ} 2$, p. 166

Pentalène, vol. $28, n^{\circ} 2$, p. 170.

Pentane, vol. 28, no 2, p. 166.

Père fondateur, vol. $28, n^{\circ} 4$, p. 325

Péremption d'instance, vol. $28, \mathrm{n}^{\circ} 2$, p. 175

Performance-compétence, vol. $28, \mathrm{n}^{\circ} 2$, p. 142

Performance, théorie de la, vol. $28, \mathrm{n}^{\circ} 2, \mathrm{p} .142$

Périphrase libre, vol. $28, n^{\circ} 1$, p. 56.

Peroxyde d'azote, vol. $28, \mathrm{n}^{\circ} 3$, p. 299.

Perspective généraviste chomskienne, vol. $28, n^{\circ} 3$, p. 299

Perte, vol. $28, n^{\circ} 2$, p. 207

Perte auditive, vol. $28, \mathrm{n}^{\circ} 2$, p. 191.

Perte de niveau, vol. $28, n^{\circ} 4$, p. 403

Perte informationnelle, vol. 28 , no 3 , p. 237.

Perturbation, vol. $28, n^{\circ} 4$, p. 366 .

Pescado, vol. $28, \mathrm{n}^{\circ} 1$, p. 53 .

Petit lexique du chauffage solaire, vol. $28, n^{\circ} 3$, p. 288.

Petite circulation, vol. $28, n^{\circ} 4$, p. 384

Petite zone partagée, vol. $28, n^{\circ} 1, p, 49$

Pez, vol. 28, no 1, p. 53.

Phase de Korotkoff, vol. $28, n^{\circ} 4$, p. 381.

Philosophie de la traduction, vol. $28, n^{\circ} 4$, p. 328.
Philosophie linguistique, vol. 28, $\mathrm{n}^{\circ} 2$, p. 136 .

Phosphore, vol. $28, \mathrm{n}^{\circ} 2$, p. 166.

Phrase, vol. $28, n^{\circ} 2$, p. 142

Phrase, structure de la, vol. 28 , no 1, p. 37

Phrase, syntaxe de la, vol. $28, \mathrm{n}^{\circ} 1, \mathrm{p} .37$.

Phrases, longueur des, vol. $28, \mathrm{n}^{\circ} 1$, p. 37.

Pièce d'identité, vol. $28, n^{\circ} 1$, p. 23 .

Pineau des Charentes, vol. $28, \mathrm{n}^{\circ} 2$, p. 184.

Piste d'asservissement, vol. $28, n^{\circ} 4$, p. 403

Piste de collecte, vol. 28, $\mathrm{n}^{\circ} 1$, p. 23.

Plan d'archives, vol. $28, n^{\circ} 4$, p. 403 .

Plan-paquet, vol. 28, no 4, p. 403.

Plan produit, vol. $28, n^{\circ} 4$, p. 403

Plan schématique de machines, vol. $28, n^{\circ} 3$, p. 305

Plan serré, vol. $28, n^{\circ} 4$, p. 403

Planche, vol. 28, no 2 , p. 196.

Plaque eutectique, vol. $28, n^{\circ} 3$, p. 290.

Plateau, vol. 28, no $^{\circ}$, p. 196.

Platine, vol. $28, n^{\circ} 2$, p. 192

Platine de magnétophone, vol. $28, \mathrm{n}^{\circ} 2$, p. 192

Platine magnétohonique, vol. 28, no 2, p. 192.

Pluperfect anglais, vol. $28, \mathrm{n}^{\circ} 3, \mathrm{p} .313$.

PNUD, vol. 28, no 1, p. 19.

Poème, vol. $28, n^{\circ} 1$, p. 41.

Poème pseudo-oriental, vol. $28, \mathrm{n}^{\circ} 4$, p. 336 .

Poignée, vol. $28, n^{\circ} 2$, p. 197.

Point de coupure, vol. $28, n^{\circ} 4$, p. 403

Pointe de style, vol. 28, no 2, p. 208.

Poiré, vol. $28, n^{\circ} 2$, p. 184 .

Pôle, vol. $28, n^{\circ} 3$, p. 280.

Politique d'authenticité, vol. $28, n^{\circ} 3$, p. 303

Politiques de traduction, vol. $28, \mathrm{n}^{\circ} 1$, p. 93 .

Polyglotte, vol. $28, n^{\circ} 4$, p. 338 .

Polyglottisme, champion du, vol. $28, n^{\circ} 4$, p. 340 .

Polynésie française, vol. $28, \mathrm{n}^{\circ} 1$, p. 17.

Polynésie occidentale, vol. $28, n^{\circ} 1$, p. 17

Polynésie orientale, vol. $28, \mathrm{n}^{\circ} 1$, p. 17.

Pompe de circulation, vol. 28, no $^{\circ} 3$, p. 290.

Pontife, vol. $28, n^{\circ} 1$, p. 44

Porte-annonces, vol. 28, no 4, p. 403

Porto, vol. 28, no 2, p. 184.

Position, indice de, vol. $28, n^{\circ} 2$, p. 167.

Positionneur de point de consigne, vol. $28, \mathrm{n}^{\circ} 4$, p. 369.

Possessif, vol. $28, n^{0} 1$, p. 51.

Post-enquête, vol. $28, n^{\circ} 4$, p. 403

Post-test, vol. $28, n^{\circ} 4$, p. 403 .

Poursuite, acte de, vol. $28, n^{\circ} 2$, p. 174

Poursuite en justice, vol. $28, \mathrm{n}^{\circ} 2$, p. 173

Pouvoir comburivore, vol. $28, n^{\circ} 3$, p. 298

Praticien, vol. 28, no 4, p. 323.

Praticien, traducteur-, vol. 28, n $^{\circ} 2$, p. 207.

Pré-enquête, vol. 28, no $^{\circ}$, p. 403.

Pré-test, vol. $28, n^{\circ} 4$, p. 404.

Préampli, vol. 28, no 2, p. 192

Préamplificateur, vol. $28, \mathrm{n}^{\circ} 2$, p. 192.

Précipitation dans un liquide, vol. $28, n^{\circ} 3$, p. 305

Précis, vol. $28, \mathrm{n}^{\circ} 3$, p. 263.

Précis terminologique, vol. $28, n^{\circ} 3$, p. 305.

Précurseur de la traduction moderne, vol. $28, \mathrm{n}^{\circ} 4$, p. 327.

Prédicat, vol. $28, \mathrm{n}^{\circ} 2$, p. 148

Prédication, vol. $28, n^{\circ} 2$, p. 148.

Premier bruit du coeur, vol. $28, n^{\circ} 4$, p. 380.

Premier harmonique, vol. $28, \mathrm{n}^{\circ} 2$, p. 192. 
Premier traducteur, vol. $28, n^{0} 4$, p. 326

Préposition, vol. 28, n० 1, p. 39.

Prescription vs description, vol. $28, \mathrm{n}^{\circ} 2$, p. 143.

Présent, vol. 28, no 3, p. 313.

Présentation, vol. 28, no 3, p. 236.

Présentation des notions, vol. $28, n^{\circ} 3$, p. 280.

Présentation, qualité de la, vol. $28, n^{\circ} 3$, p. 239

Présentation systématique, vol. $28, \mathrm{n}^{\circ} 3$, p. 280 ; vol. $28, n^{\circ} 3$, p. 282.

Présentoir, vol. 28, n $^{\circ} 4$, p. 403 .

Pression, vol. 28 , no 3 , p. 298

Pression artérielle, vol. $28, n^{\circ} 4$, p. 377.

Prêt-à-monter, vol. 28, no 4, p. 403.

Préventif, vol. $28, n^{\circ} 2$, p. 162.

Prévention, vol. 28, n० 2, p. 162.

Prévoir, vol. $28, n^{\circ} 3$, p. 269.

Primauté de l'esprit, vol. $28, n^{\circ} 4$, p. 327.

Principe de soumission-subversion, vol. $28, \mathrm{n}^{\circ} 1$, p. 43.

Principe fondamental, vol. $28, n^{\circ} 4$, p. 328.

Problématique du mot, vol. 28 , no 3 , p. 299.

Problème de culture, vol. $28, n^{\circ} 2$, p. 133 .

Problème de matière, vol. 28 , no 2 , p. 134

Problème sémantique, vol. $28, n^{\circ} 1$, p. 26.

Problème socio-culturel, vol. 28 , no 1, p. 25.

Procédé, vol. $28, \mathrm{n}^{\circ} 2$, p. 175

Procédure, vol. 28 , no 2 , p. 173 ; vol. $28, n^{\circ} 2$, p. 175.

Procédure, acte de, vol. $28, \mathrm{n}^{\circ} 2$, p. 175.

Procédure, faire acte de, vol. 28, no 2, p. 174.

Procès, vol. $28, n^{\circ} 2$, p. 176.

Procès verbal, vol. $28, \mathrm{n}^{\circ} 2$, p. 176

Processus, vol. $28, n^{\circ} 4$, p. 365

Processus industriel, vol. $28, n^{\circ} 3$, p. 305.

Processus traductionnel, vol. 28, no 4, p. 323.

Production de gaz de remplacement, vol. $28, n^{\circ} 3$, p. 297.

Professeur de langues, vol. 28, no 3, p. 311.

Profil d'intérêt, vol. $28, n^{\circ} 2$, p. 218 .

Profil documentaire, vol. $28, \mathrm{n}^{\circ} 2$, p. 218.

Profils, index des, vol. $28, n^{\circ} 2$, p. 218

Programme de formation, vol. $28, \mathrm{n}^{\circ} 1$, p. 26.

Progression dans le temps, vol. $28, n^{\circ} 1$, p. 49.

Projet de loi, vol. 28, no $^{\circ}$, p. 371.

Promo, vol. 28 , no 4 , p. 404.

Promotion du français, vol. $28, n^{\circ} 1$, p. 92

Pronom, vol. $28, \mathrm{n}^{\circ} 1$, p. 50.

Prophète, vol. 28, n०2, p. 162.

Prophétie, vol. $28, n^{\circ} 2$, p. 162

Propre, vol. $28, n^{\circ} 4$, p. 404.

Propriété physico-chimique, vol. $28, \mathrm{n}^{\circ} 2$, p. 167.

Province, vol. 28, no 3, p. 260.

Prune, vol. 28, n०2, p. 185.

Psycholinguistique, vol. $28, n^{\circ} 1$, p. 48

Publicitaire, vol. $28, n^{\circ} 4$, p. 404.

Publipostage, vol. 28 , no 4 , p. 404.

Puissance crête à crête, vol. 28, n $^{\circ} 2$, p. 192.

Puissance de crête, vol. $28, n^{\circ} 2$, p. 192

Puissance de la traduction, vol. $28, \mathrm{n}^{\circ} 4$, p. 343

Puissance musicale, vol. 28, $\mathrm{n}^{\circ} 2$, p. 192

Punch, vol. 28, no 2 , p. 185.

Purine, vol. 28, n$^{\circ} 2$, p. 170.
Qualité de la présentation, vol. 28, n $^{\circ} 3$, p. 239.

Qualité de l'interprétation, vol. 28, no 3, p. 239.

Qualité du travail, vol. $28, \mathrm{n}^{\circ} 3$, p. 239.

Qualité du travail en interprétation, vol. $28, n^{\circ} 3$, p. 236 .

Quantification, vol. 28, no 4, p. 369.

Quater-, vol. $28, \mathrm{n}^{\circ} 2$, p. 171.

Quaternaphtalène, vol. $28, \mathrm{n}^{\circ} 2, \mathrm{p} .171$.

Quaterni-, vol. 28, n०2, p. 171

Quaternihexalane, vol. $28, \mathrm{n}^{\circ} 2$, p. 171 .

Querelle doctrinale, vol. $28, \mathrm{n}^{\circ} 4$, p. 324 .

Querer, vol. 28, no 1, p. 37.

Queue naturelle, vol. $28, n^{\circ} 4$, p. 362.

Quinine, vol. 28, n०2, p. 166.

Quotidienneté langagière, vol. $28, n^{\circ} 1$, p. 40.

Radiocassette, vol. 28 , no 4, p. 404

Rang, vol. 28, no 3, p. 293.

Rang-échelle, vol. 28, n $^{\circ} 2$, p. 143.

Rapport de passation de service, vol. $28, \mathrm{n}^{\circ} 1$, p. 23.

Rapport S/B, vol. 28, n०2, p. 192.

Rapport signal/bruit, vol. 28, no $^{\circ}$, p. 192.

Raquette, vol. $28, n^{\circ} 2$, p. 197 ; vol. $28, n^{\circ} 4$, p. 358 .

Rareté-affectivité, vol. $28, \mathrm{n}^{\circ} 1$, p. 40.

Ré-écriture, vol. $28, n^{\circ} 1$, p. 43.

Ré-énonciation, vol. $28, \mathrm{n}^{\mathrm{O}} 1$, p. 45

Réaction d'addition, vol. $28, \mathrm{n}^{\mathrm{0}} 2$, p. 168.

Réaction de substitution, vol. $28, \mathrm{n}^{\circ} 2, \mathrm{p} .166$

Recensement, exhaustivité du, vol. 28, n $^{\circ} 3$, p. 280.

Récepteur, vol. $28, n^{\circ} 3$, p. 237.

Récepteur alpha, vol. 28 , no 4 , p. 388

Récepteur bêta, vol. 28 , no 4, p. 388 .

Réception, vol. $28, n^{\circ} 3$, p. 237

Receveur en chef, vol. $28, n^{\circ} 1$, p. 23.

Recherche, fichier, vol. $28, n^{\circ} 2$, p. 215.

Recherche thématique, vol. $28, n^{\circ} 4$, p. 392.

Reconstitution intégrale du discours, vol. $28, n^{\circ} 3$, p. 242.

Recoupement, vol. 28, no 3, p. 280.

Recueil des évaluations, vol. $28, n^{\circ} 3$, p. 242.

Recycler, vol. $28, n^{\circ} 3$, p. 258.

Rédaction, vol. 28 , no 1 , p. 24.

Redondance, règle de, vol. $28, n^{\circ} 2$, p. 158.

Réduction, vol. 28, no 3, p. 299.

Réduction en copeaux, vol. $28, n^{\circ} 3$, p. 305.

Référent, vol. $28, \mathrm{n}^{\circ} 2$, p. 133.

Réflexion, vol. 28 , n०2, p. 161

Reformulation, vol. 28 , no 2 , p. 133.

Régisseur de distribution, vol. $28, n^{0} 4$, p. 404.

Règle d'allomorphie, vol. $28, \mathrm{n}^{\circ} 2$, p. 163 .

Règle de formation de mot, vol. $28, \mathrm{n}^{\circ} 2, \mathrm{p} .163$.

Règle de formation de mots, vol. $28, n^{\circ} 2$, p. 158

Règle de formation de radicaux, vol. $28, n^{\circ} 2$ p. 157.

Règle de redondance, vol. $28, \mathrm{n}^{\circ} 2$, p. 158

Règle de troncation, vol. 28 , no 2 , p. 157 ; vol. 28 , $\mathrm{n}^{\circ} 2$, p. 163

Réglementée, vol. $28, n^{\circ} 2$, p. 185.

Regroupement des notions, vol. $28, n^{\circ} 3$, p. 280.

Régulateur, vol. $28, n^{\circ} 4$, p. 367.

Régulateur numérique centralisé, vol. $28, n^{\circ} 4$, p. 369 . 
Régulation, vol. 28 , no 4 , p. 365 .

Régulation analogique, vol. $28, n^{\circ} 4$, p. 367.

Régulation avec action anticipatrice, vol. $28, n^{\circ} 4$, p. 366.

Régulation avec action prévisionnelle, vol. 28 , n० 4, p. 366.

Régulation de correspondance, vol. $28, n^{\circ} 4$, p. 366 .

Régulation de maintien, vol. 28 , no 4 , p. 366.

Régulation numérique, vol. $28, n^{\circ} 4$, p. 367 .

Régulation numérique directe, vol. $28, \mathrm{n}^{\circ} 4$ p. 367 ; vol. 28, n $^{\circ} 4$, p. 369 .

Régulation numérique indirecte, vol. $28, n^{\circ} 4$ p. 367.

Régulation par réaction, vol. $28, \mathrm{n}^{\circ} 4, \mathrm{p} .366$.

Régulation prédictive, vol. 28 , n० 4 , p. 366 .

Rei, vol. 28 , no 1 , p. 36 .

Relief sonore, vol. $28, n^{\circ} 2$, p. 191

Remarques générales de traduction, vol. 28 , $\mathrm{n}^{\circ} 1$, p. 26.

Remplacement, nomenclature par, vol. $28, n^{\circ} 2$, p. 169 .

Remue-méninges, vol. $28, n^{\circ} 4$, p. 404.

Renard, vol. $28, \mathrm{n}^{\circ} 2$, p. 194.

Rendement de conversion, vol. $28, n^{\circ} 3$, p. 290

Renfort de bois, vol. 28, n $^{\circ} 2$, p. 192.

Renfort métallique, vol. $28, \mathrm{n}^{\circ} 2$, p. 192

Renvoi, vol. 28 , no 3 , p. 280 ; vol. $28, n^{\circ} 4$, p. 370

Repérage, vol. $28, n^{\circ} 3$, p. 280 .

Repérage, défaut d'homogénéité dans le, vol. 28 , $n^{\circ} 3$, p. 280.

Repérage, homogénéité dans le, vol. 28 , no 3 , p. 280.

Répétition, vol. $28, \mathrm{n}^{\circ} 1$, p. 38

Reportage électronique, vol. 28 , no 4, p. 404.

Réseau, vol. 28 , no 1 , p. 54

Réseau de terminologie du gouvernement canadien, vol. $28, n^{\circ} 2$, p. 216.

Réservoir de stockage, vol. $28, n^{\circ} 3$, p. 290.

Résonance culturelle, vol. $28, \mathrm{n}^{\circ} 1$, p. 41 .

Résonance, fréquence de, vol. $28, \mathrm{n}^{\circ} 2$, p. 191.

Respect de la lettre, vol. $28, n^{\circ} 4$, p. 326.

Resserrer un montage, vol. 28 , no 4 , p. 404

Restriction, vol. $28, \mathrm{n}^{\circ} 3$, p. 303 .

Retard, vol. $28, n^{\circ} 4$, p. 367.

Retour en arrière, vol. $28, n^{\circ} 4$, p. 404.

Retour sonore, vol. 28 , no 1 , p. 41

Retrait, vol. $28, n^{\circ} 3$, p. 293.

Réunification des deux Camerouns, vol. 28, no 1 , p. 20.

Revêtement, vol. 28, no 3, p. 292.

Revêtement antireflet, vol. $28, \mathrm{n}^{\circ} 3$, p. 290.

Revêtement sélectif, vol. $28, \mathrm{n}^{\circ} 3$, p. 290.

Réviseur camerounais, vol. $28, \mathrm{n}^{\circ} 1$, p. 26.

Réviseur d'organisations internationales, vol. 28 , no 1 , p. 90 .

Rhum, vol. $28, \mathrm{n}^{\circ} 2$, p. 185.

Rime externe, vol. $28, n^{\circ} 1$, p. 41.

Rime interne, vol, $28, \mathrm{n}^{\circ} 1$, p. 41.

R.M.S., vol. 28 , no 2 , p. 192 .

Roche couverture, vol. 28 , no 3, p. 299.

Rôle de l'interprète, vol. $28, n^{\circ} 1$, p. 94.

Ronde enfantine, vol. 28 , no 1 , p. 41 .

Rotophaseur, vol. 28 , no 4 , p. 404.

Roubar, vol. 28, n $^{\circ} 1$, p. 37.

Route non asphaltée, vol. $28, \mathrm{n}^{\circ} 1$, p. 23.

Royaume de Tonga, vol. $28, \mathrm{n}^{\circ} 1$, p. 17.

Saint Jérôme, vol. $28, n^{\circ} 1$, p. 36 .

Salle d'écoute, vol. 28 , no 2 , p. 192.

Sang artériel, vol. $28, n^{\circ} 4$, p. 383.

Sang veineux, vol. 28 , no 4 , p. 383 .

Sauveteur, vol. $28, n^{\circ} 2$, p. 160 .

Savoir linguistique, le, vol. $28, n^{\circ} 1$, p. 48

Scanneur, vol. 28 , no 4 , p. 404 .

Scéarimage, vol. $28, n^{\circ} 4$, p. 404 .

SCFA, vol. $28, n^{\circ} 4$, p. 417.

Schéma arborescent, vol. 28, n $^{\circ} 3$, p. 280.

Schéma détaillé de la traduction, vol. $28, n^{\circ} 2$, p. 206.

Schéma fonctionnel de processus, vol. $28, \mathrm{n}^{\circ} 3$, p. 305.

Sciage cintré, vol. 28 , no 4 , p. 358 .

Sciage courbe, vol. $28, n^{\circ} 2$, p. 195.

Sciage de finition, vol. 28, no $^{\circ}$, p. 196.

Sciage, outil manuel de, vol. 28, n० 2, p. 192.

Sciage sinueux, vol. $28, \mathrm{n}^{\circ} 2$, p. 195.

Scie, vol. $28, \mathrm{n}^{\circ} 2$, p. 192

Scie à araser, vol. $28, n^{\circ} 2$, p. 197 ; vol. $28, n^{\circ} 4$, p. 359.

Scie à araser de côté, vol. $28, n^{\circ} 2$, p. 197 ; vol. 28 , $n^{\circ} 4$, p. 361 .

Scie à arc, vol. 28, no $^{\circ}$, p. 197 ; vol. $28, n^{\circ} 4$ p. 363.

Scie à archet, vol. $28, n^{\circ} 4$, p. 363 ; vol. 28 , no 4 , p. 363.

Scie à bras, vol. $28, \mathrm{n}^{\circ} 2$, p. 198.

Scie à bûches, vol. $28, n^{\circ} 2$, p. 197 ; vol. $28, n^{\circ} 4$, p. 362 ; vol. $28, n^{\circ} 4$, p. 363 .

Scie à bûches savoyarde, vol. $28, n^{\circ} 4$, p. 362 .

Scie à cadre, vol. $28, n^{\circ} 2$, p. 196 ; vol. 28 , n० 4 , p. 363 .

Scie à cadre du tonnelier, vol. 28, no $^{\circ} 2$, p. 197 ; vol. $28, n^{\circ} 4$, p. 362

Scie à cadre métallique, vol. 28, n $^{\circ} 2$, p. 194.

Scie à chantourner, vol. $28, n^{\circ} 2$, p. 197 ; vol. 28 , $n^{\circ} 4$, p. 361 ; vol. 28 , no 4 , p. 362 ; vol. $28, n^{\circ} 4$, p. 364.

Scie à chantourner du bijoutier, vol. $28, \mathrm{n}^{\circ} 2$ p. 197 ; vol. $28, n^{\circ} 4$, p. 365 .

Scie à châsse, vol. 28, no $^{\circ}$, p. 199

Scie à châssis, vol. 28, n $^{\circ} 2$, p. 196

Scie à débit, vol. 28, n $^{\circ} 4$, p. 359.

Scie à débiter, vol. $28, n^{\circ} 2$, p. 197 ; vol. $28, n^{\circ} 4$, p. 359.

Scie à découper, vol. 28, no $^{\circ}$, p. 194 ; vol. 28 , $n^{\circ} 2$, p. 197 ; vol. 28 , no 4 , p. 363 .

Scie à deux poignées, vol. 28 , n० 2, p. 192.

Scie à dos arqué, vol. 28, n $^{\circ} 2$, p. 194

Scie à dos droit, vol. $28, \mathrm{n}^{\circ} 2$, p. 194.

Scie à double lame, vol. $28, n^{\circ} 2$, p. 197 ; vol. 28 , $\mathrm{n}^{\circ} 4$, p. 361.

Scie à enraser, vol. $28, n^{\circ} 4$, p. 359.

Scie à fer, vol. $28, n^{\circ} 4$, p. 365 .

Scie à main, vol. $28, n^{\circ} 2$, p. 192.

Scie à métaux, vol. $28, n^{\circ} 2$, p. 197 ; vol. $28, n^{\circ} 4$, p. 365 .

Scie à monture, vol. $28, n^{\circ} 2$, p. 192 ; vol. $28, n^{\circ} 2$, p. 196.

Scie à poignée, vol. $28, n^{\circ} 2$, p. 192 
Scie à raser, vol. 28, no $^{\circ}$, p. 359.

Série énumérative, vol. $28, \mathrm{n}^{\circ} 2, \mathrm{p} .170$.

Scie à refendre, vol. $28, n^{\circ} 2$, p. 197 ; vol. $28, n^{\circ} 4$, Série multiplicative, vol. $28, n^{\circ} 2$, p. 170 p. 361 .

Scie à tenon, vol. $28, n^{\circ} 2$, p. 197 ; vol. $28, n^{\circ} 4$, p. 359.

Scie à tenons, vol. $28, \mathrm{n}^{\circ} 4$, p. 359.

Scie à tourner, vol. 28, n $^{\circ} 4$, p. 361 .

Scie allemande, vol. 28 , no 4 , p. 361 .

Scie bocfil, vol. $28, n^{\circ} 4$, p. 363 .

Scie d'assemblage, vol. $28, n^{\circ} 4$, p. 361

Scie de bijoutier, vol. $28, n^{\circ} 4$, p. 365 .

Scie de charpentier, vol. 28 , n० 2 , p. 197 ; vol. 28 , no 4 , p. 358 .

Scie de débit, vol. 28 , no 4 , p. 359

Scie de long, vol. $28, n^{\circ} 2$, p. 194 ; vol. $28, n^{\circ} 2$, p. 197.

Scie de marquetterie, vol. $28, \mathrm{n}^{\circ} 4$, p. 363 .

Scie de menuisier, vol. $28, n^{\circ} 2$, p. 196.

Scie de taille du charpentier, vol. 28 , no 4 , p. 358 .

Scie de travers, vol. $28, n^{\circ} 2$, p. 195 ; vol. $28, n^{\circ} 4$, p. 358 .

Scie de travers, grande, vol. $28, n^{\circ} 2$, p. 197.

Scie du scieur de long, vol. $28, n^{\circ} 2$, p. 198.

Scie manoeuvrée par deux hommes, vol. $28, n^{\circ} 4$, p. 358 .

Scie manuelle, vol. $28, n^{\circ} 2$, p. 192 ; vol. $28, n^{\circ} 4$, p. 358.

Scie montée, vol. 28 , no 2 , p. 196.

Scie raquette, vol. $28, n^{\circ} 4$, p. 358

Scie renforcée, vol. $28, \mathrm{n}^{\circ} 2$, p. 192.

Scie sur cadre, vol. $28, n^{\circ} 2$, p. 196.

Scie tournante, vol. $28, n^{\circ} 4$, p. 361 .

Scie violon, vol. 28, n $^{\circ} 4$, p. 358 .

Sciotte, vol. 28 , no 4 , p. 363 .

Scripte, vol. $28, n^{\circ} 4$, p. 404.

Scrutateur, vol. $28, \mathrm{n}^{\circ} 2$, p. 160.

Sec, vol. 28 , no 4 , p. 404.

Secondaire, le, vol. 28, n $^{\circ} 3$, p. 310.

Secrétaire général, vol. $28, n^{\circ} 1$, p. 23.

Secteur, vol. $28, n^{\circ} 2$, p. 162.

Section, vol. $28, n^{\circ} 2$, p. 162.

Section de langue anglaise, vol. $28, n^{\circ} 1$, p. 21.

Section de langue française, vol. $28, n^{\circ} 1$, p. 21

Sectionnement, vol. $28, \mathrm{n}^{\circ} 2$, p. 196.

Sel, vol. $28, n^{\circ} 2$, p. 140.

Sel eutectique, vol. 28 , no 3 , p. 290.

Sélectionné, vol. 28 , no 4 , p. 404.

Sémantique et traduction, vol. $28, \mathrm{n}^{\circ} 1$, p. 52.

Sémantique moderne, vol. $28, n^{\circ} 2$, p. 141.

Sémantique, unité, vol. $28, n^{\circ} 2$, p. 149.

Sémasiologie, vol. $28, \mathrm{n}^{\circ} 2$, p. 133 .

Sème contextuel, vol. 28, n $^{\circ} 1$, p. 52 .

Sème générique, vol. $28, n^{\circ} 1$, p. 53 .

Sème spécifique, vol. $28, n^{\circ} 1$, p. 53 .

Sème virtuel, vol. 28 , no 1 , p. 53 .

Sémène, vol. $28, n^{\circ} 1$, p. 52 .

Sens, vol. $28, n^{\circ} 1$, p. 52 ; vol. $28, n^{\circ} 2$, p. 162.

Sens apparent, vol. $28, n^{\circ} 2$, p. 149.

Sens, correspondance, vol. $28, \mathrm{n}^{\circ} 2$, p. 133.

Sens, doué de, vol. 28, no $^{\circ}$, p. 140.

Sens et forme, vol. $28, \mathrm{n}^{\circ} 2$, p. 141

Sens réel, vol. $28, n^{\circ} 2$, p. 149.

Sentir, vol. $28, n^{\circ} 2$, p. 162.

Séquence syllabique inédite, vol. 28 , no 3 , p. 299

Série conjonctive, vol. $28, n^{\circ} 2$, p. 171 .

Série distributive, vol. $28, \mathrm{n}^{\circ} 2$, p. 171 .

Serré, vol. $28, n^{\circ} 4$, p. 404.

Service de néologie et de terminologie, vol. 28 , no 1, p. 92.

Service de traduction, vol. $28, \mathrm{n}^{\circ} 1$, p. 22.

Service linguistique, vol. 28 , no 1, p. 22 .

Serviteur, vol. 28 , no 2 , p. 160 .

SFT, vol. $28, n^{\circ} 1$, p. 90.

Siglaison, vol. 28, no $^{\circ}$, p. 299.

Sigle d'organisation, vol. 28, n $^{\circ} 3$, p. 298.

Signal de commande, vol. $28, n^{\circ} 4$, p. 369.

Signal de consigne, vol. $28, n^{\circ} 4$, p. 369.

Signal de correction, vol. 28 , no 4 , p. 367 .

Signalisation routière, vol. 28 , no 1 , p. 50 .

Signe, vol. 28 , no 2 , p. 141.

Signifiant, vol. $28, n^{\circ} 3$, p. 283 ; vol. $28, n^{\circ} 4$, p. 325 .

Significatif, vol. $28, \mathrm{n}^{\circ} 2$, p. 140 .

Signification, vol. 28, n $^{\circ} 1$, p. 52 ; vol. $28, n^{\circ} 1$, p. 54.

Signification latente, vol. $28, n^{\circ} 1$, p. 56.

Signification linguistique, vol. 28 , no 2, p. 141 .

Signification politico-philosophique, vol. $28, \mathrm{n}^{\circ} 1$, p. 44.

Signification pragmatique, vol. 28, no 2 , p. 205.

Signifié, vol. $28, n^{\circ} 2$, p. 145 ; vol. $28, n^{\circ} 3$, p. 283 ; vol. $28, n^{\circ} 4$, p. 325 .

Silencieux, vol. $28, \mathrm{n}^{\circ} 4$, p. 405.

SILF, vol. $28, n^{\circ} 4$, p. 431

Situation, vol. 28, no $^{\mathrm{o}} 1$, p. 23.

Situation, contexte de, vol. 28 , no 2 , p. 130.

Situationnel, contexte, vol. $28, \mathrm{n}^{\circ} 2$, p. 137 .

Société française des traducteurs, vol. $28, n^{\circ} 1$, p. 90 .

Socio-linguistique, vol. $28, \mathrm{n}^{\circ} 1$, p. 48

Soi-disant, vol. $28, n^{\circ} 1$, p. 54 .

Solarisation, vol. 28, no 3, p. 290.

Solution tampon, vol. 28 , n० 3 , p. 305.

Sommier, vol. 28 , no 2 , p. 196.

Son fondamental, vol. $28, n^{\circ} 2$, p. 192.

Son harmonique, vol. $28, n^{\circ} 2$, p. 191 .

Son original, vol. $28, \mathrm{n}^{\circ} 2$, p. 192.

Sonal, vol. $28, n^{\circ} 4$, p. 405 .

Sonorisation, vol. 28 , no 4 , p. 405.

Sortie, vol. 28 , no 4 , p. 366.

Souche, vol. 28, n $^{\circ} 4$, p. 405.

Souffle, vol. $28, n^{\circ} 4$, p. 381

Soufre, vol. 28 , no 2 , p. 166.

Soumission-subversion, vol. $28, n^{\circ} 1$, p. 43.

Source sonore, vol. $28, \mathrm{n}^{\mathrm{0}} 2$, p. 192.

South Pacific Bureau for Economic Cooperation, vol. 28, n $^{\circ} 1$, p. 19.

SPEC, vol. 28, no $^{\circ}$, p. 19.

Spécialiste de domaine, vol. 28, no 3, p. 279.

Spécifique, vol. 28, no $^{\circ}$, p. 299.

Sphère de signification, vol. $28, n^{\circ} 1$, p. 49

Spiritueux, vol. 28, n $^{\circ} 2$, p. 179.

Stéréophonie, vol. 28, n $^{\circ} 2$, p. 192.

Stérile, vol. 28, n $^{\circ} 3$, p. 257 .

Stockage à court terme, vol. $28, \mathrm{n}^{\circ} 3$, p. 290.

Stockage à lit de cailloux, vol. $28, \mathrm{n}^{\mathrm{o}} 3$, p. 291 .

Stockage à lit de galets, vol. 28 , no 3 , p. 291 .

Stockage à lit de roches, vol. $28, n^{\circ} 3$, p. 291.

Stockage court, vol. 28, no 3, p. 291.

Stockage de courte durée, vol. 28, n$^{\circ} 3$, p. 291. 
Stockage de longue durée, vol. 28, no 3, p. 291.

Stockage intersaisonnier, vol. $28, n^{\circ} 3$, p. 291.

Stockage journalier, vol. $28, n^{\circ} 3$, p. 291

Stockage quotidien, vol. $28, \mathrm{n}^{\circ} 3$, p. 291

Stockage thermique solaire, vol. $28, \mathrm{n}^{\mathrm{0}} 3$, p. 291.

Stratégie de la déchirure, vol. $28, \mathrm{n}^{\circ} 2$, p. 208

Stratford, Philip, vol. 28, n॰ 4, p. 356.

Structure alvéolaire sélective, vol. $28, n^{\circ} 3$, p. 291.

Structure anti-perte, vol. $28, n^{\circ} 3$, p. 291.

Structure arborescente, vol. $28, n^{\circ} 3$, p. 280.

Structure cellulaire antirayonnante, vol. $28, n^{\circ} 3$, p. 291.

Structure de Francia, vol. 28, no 3, p. 291.

Structure de la phrase, vol. 28 , no 1, p. 37

Structure de nid d'abeilles, vol. 28 , n० 3, p. 291

Structure méthodologique, vol. $28, n^{\circ} 4$, p. 332 .

Structure profonde, vol. $28, \mathrm{n}^{\circ} 1$, p. 51 ; vol. 28 , $n^{\circ} 2$, p. 148

Structure superficielle, vol. $28, \mathrm{n}^{\circ} 2$, p. 148.

Style indirect, vol. $28, \mathrm{n}^{\circ} 3$, p. 250.

Style indirect libre, vol. $28, n^{\circ} 3$, p. 250

Style, pointe de, vol. $28, \mathrm{n}^{\circ} 2$, p. 208.

Stylicien, vol. $28, n^{\circ} 4$, p. 405 .

Stylicienne, vol. $28, n^{\circ} 4$, p. 405

Stylique, vol. $28, n^{\circ} 4$, p. 405 .

Stylisme, vol. $28, n^{\circ} 4$, p. 405 .

Styliste, vol. $28, n^{\circ} 4$, p. 405.

Stylistique comparée, vol 28 no 3 , p. 313 ; vol 28 $n^{\circ} 4$, p. 417.

Substance, vol. $28, \mathrm{n}^{\circ} 2$, p. 142

Substituant, vol. $28, n^{\circ} 2$, p. 167.

Substituant fonctionnel, vol. $28, \mathrm{n}^{\circ} 2$, p. 167.

Substituant inorganique, vol. $28, n^{\circ} 2$, p. 167

Substitution, réaction de, vol. $28, \mathrm{n}^{\circ} 2$, p. 166.

Substrat local, vol. $28, \mathrm{n}^{\circ} 3$, p. 303.

Suffixation, vol. $28, n^{\circ} 3$, p. 299.

Suffixe-ateur, vol. $28, n^{\circ} 2$, p. 157.

Sujet apparent, vol. $28, \mathrm{n}^{\circ} 2$, p. 149.

Sujet grammatical, vol. $28, \mathrm{n}^{\circ} 2$, p. 149

Sujet logique, vol. $28, n^{\circ} 2$, p. 149.

Sujet réel, vol. $28, n^{\circ} 2$, p. 149

Sulfure, vol. $28, \mathrm{n}^{\circ} 2$, p. 168.

Superordonné, vol. $28, n^{\circ} 4$, p. 395.

Superposition totale, vol. 28 , no 1, p. 49

Supplétif, vol. $28, \mathrm{n}^{\circ} 2$, p. 162.

Supplétion, vol. $28, \mathrm{n}^{\circ} 2$, p. 162.

Suramplificateur, vol. $28, n^{\circ} 4$, p. 405

Surdité relative, vol. $28, n^{\circ} 2$, p. 191.

Surface absorbante, vol. $28, n^{\circ} 3$, p. 291

Surface sélective, vol. $28, n^{\circ} 3$, p. 291.

Syndrome de la crosse aortique, vol. $28, n^{\circ} 4$, p. 384.

Synopsis, vol. $28, n^{\circ} 2$, p. 162.

Synoptique, vol. $28, n^{\circ} 2$, p. 162

Syntaxe brésilienne, vol. $28, n^{\circ} 1$, p. 37

Syntaxe de la phrase, vol. $28, n^{\circ} 1$, p. 37

Synthèse, vol. $28, n^{\circ} 3$, p. 242.

Syntonisateur, vol. 28, no 4, p. 405.

Syntoniseur, vol. $28, n^{\circ} 4$, p. 405.

Système de classification, vol. $28, n^{\circ} 4$, p. 395 .

Système de concepts, vol. $28, n^{\circ} 4$, p. 393 .

Système lymphatique, vol. $28, n^{\circ} 4$, p. 379 .

Système notionnel, vol. $28, n^{\circ} 3$, p. 280 .

Système rénine-angiotensine, vol. $28, n^{\circ} 4$, p. 379

Système solaire actif, vol. $28, n^{\circ} 3$, p. 291

Système solaire mécanisé, vol. $28, \mathrm{n}^{\circ} 3$, p. 291.
Système solaire naturel, vol. $28, \mathrm{n}^{\circ} 3$, p. 291.

Système solaire passif, vol. $28, n^{\circ} 3$, p. 291.

Systémique, vol. $28, \mathrm{n}^{\circ} 4$, p. 385 .

Systole auriculaire, vol. $28, \mathrm{n}^{\mathrm{0}} 4$, p. 377.

Systole ventriculaire, vol. 28, no 4, p. 377

t-eur, vol. $28, \mathrm{n}^{\circ} 2$, p. 157

t-ion, vol. $28, \mathrm{n}^{\circ} 2$, p. 157

Table de lecture, vol. $28, n^{\circ} 2$, p. 192.

Taffia, vol. $28, n^{\circ} 2$, p. 185 .

Tamis linguistique, vol. $28, \mathrm{n}^{\circ} 1$, p. 52.

Tapioca, vol. $28, \mathrm{n}^{\circ} 1$, p. 25.

Taux d'aération, vol. 28, no $^{\circ}$, p. 298.

Taux de distorsion, vol. $28, \mathrm{n}^{\circ} 2$, p. 192

Taxinomie, vol. $28, n^{\circ} 1$, p. 56 .

Technicien du langage, vol. $28, n^{\circ} 2$, p. 134

Technologie solaire, vol. $28, n^{\circ} 3$, p. 283.

Teetotalisme, vol. $28, n^{\circ} 2$, p. 180.

Télésouffleur, vol. $28, n^{\circ} 4$, p. 405 .

Télévisio à accès conditionnel, vol. $28, n^{\circ} 4$, p. 405.

Télévision à péage, vol. $28, n^{\circ} 4$, p. 405 .

Télévision payante, vol. $28, n^{\circ} 4$, p. 405 .

Température de fonctionnement, vol. $28, n^{\circ} 3$, p. 291.

Température d'équilibre, vol. $28, \mathrm{n}^{\circ} 3$, p. 291

Température d'exploitation, vol. $28, \mathrm{n}^{\circ} 3, \mathrm{p} .291$.

Température limite, vol. $28, n^{\circ} 3$, p. 292.

Temps de montée, vol. $28, \mathrm{n}^{\circ} 2, \mathrm{p} .192$.

Temps verbaux, vol. $28, \mathrm{n}^{\circ} 1$, p. 38.

Teneur, vol. $28, n^{\circ} 2$, p. 132.

Tenon, joue de, vol. $28, n^{\circ} 2$, p. 196.

Tension artérielle, vol. $28, \mathrm{n}^{\circ} 4$, p. 376 ; vol. 28 , $n^{\circ} 4$, p. 377.

Tension, corde de, vol. 28, no $^{\circ} 2$, p. 196.

Tentateur, vol. $28, \mathrm{n}^{\circ} 2$, p. 160.

Tentation, vol. $28, \mathrm{n}^{\circ} 2$, p. 160 .

Tentative, vol. $28, n^{\circ} 2$, p. 160 .

Tenter, vol. 28, no $^{\circ}$, p. 160.

Tenue, vol. $28, \mathrm{n}^{\circ} 3$, p. 294.

Ter-, vol. $28, \mathrm{n}^{\circ} 2$, p. 171 .

Tercyclopropane, vol. $28, n^{0} 2$, p. 171

Terminographe, vol. $28, \mathrm{n}^{\circ} 3$, p. 279.

Terminologie, vol. $28, n^{\circ} 4$, p. 391

Terminologie bilingue, vol. $28, \mathrm{n}^{\circ} 3$, p. 280

Terminologie de l'amiante, vol. $28, \mathrm{n}^{\circ} 3$, p. 282.

Terminologie, fichier, vol. $28, \mathrm{n}^{\circ} 2$, p. 215 .

Terminologie linguistique, vol. $28, n^{\circ} 2$, p. 150

Terminologie, réseau de, vol. $28, \mathrm{n}^{\circ} 2$, p. 216.

Terminologiste, vol. $28, n^{\circ} 2$, p. 151.

Termium I, vol. $28, \mathrm{n}^{\circ} 2$, p. 215 .

Terni-, vol. $28, n^{\circ} 2$, p. 171.

Test aveugle, vol. $28, n^{\circ} 4$, p. 405

Test de porte-annonces, vol. 28, $n^{\circ} 4$, p. 406

Test d'évaluation, vol. $28, n^{\circ} 4$, p. 406.

Test du lendemain, vol. $28, n^{\circ} 4$, p. 406.

Test sur tirage équifractionné, vol. $28, n^{\circ} 4$, p. 406.

Tête de lecture, vol. $28, n^{\circ} 2$, p. 192.

Tétra-, vol. $28, n^{\circ} 2$, p. 170 .

Tétrahydro-1,2,3,4 naphtalène, vol. $28, n^{\circ} 2$, p. 168 .

Tétrakis-, vol. $28, \mathrm{n}^{\circ} 2$, p. 170

-teur, vol. $28, n^{\circ} 2$, p. 159

Texte entier, vol. 28, n $^{\circ} 2$, p. 142.

Texte, lecture du, vol. 28, no 2, p. 207. 
Texte, traduction de, vol. $28, n^{\circ} 2$, p. 149.

Textualité, vol. $28, n^{\circ} 1$, p. 48 .

Thème, vol. $28, \mathrm{n}^{\circ} 3$, p. 312 .

Thème d'imitation, vol. 28 , no 3 , p. 313

Théorie de la dérivation, vol. $28, \mathrm{n}^{\circ} 4$, p. 331 .

Théorie de la performance, vol. $28, \mathrm{n}^{\circ} 2$, p. 142.

Théorie de la traduction, vol. $28, \mathrm{n}^{\circ} 1$, p. 94.

Théorie des noyaux de glace, vol. $28, \mathrm{n}^{\circ} 3$, p. 25

Théorie générale, vol. $28, \mathrm{n}^{\circ} 4$, p. 323 .

Théorie générale de la traduction, vol. $28, \mathrm{n}^{\circ} 4$, p. 323 ; vol. 28 , no 4 , p. 324 ; vol. 28 , no 4 , p. 328 .

Théorie linguistique, vol. $28, n^{\circ} 4$, p. 324

Théoritrad, vol. $28, n^{\circ} 4$, p. 324 .

Thia-, vol. 28, no $^{\circ}$, p. 169

Tic, vol. $28, \mathrm{n}^{\mathrm{O}} 3$, p. 241

tif, vol. $28, n^{\circ} 2$, p. 159 .

Timbre, vol. 28, no 2 , p. 192.

-tion, vol. $28, \mathrm{n}^{\circ} 2$, p. 159.

Tirage équifractionné, vol. $28, n^{\circ} 4$, p. 406

Tirer une flèche, vol. $28, n^{\circ} 3$, p. 303 .

Titres et appellations officielles, fichier, vol. 28 $\mathrm{n}^{\circ} 2$, p. 215 .

toire, vol. $28, n^{\circ} 2$, p. 159.

Toit capteur, vol. $28, n^{\circ} 3$, p. 291

Torche, vol. $28, \mathrm{n}^{\circ} 3$, p. 297.

Tourillon, vol. 28 , no 4 , p. 361.

Tourne-disque, vol. $28, \mathrm{n}^{\circ} 2$, p. 192

Tournure, vol. 28 , no 3 , p. 241

Tournure moyenâgeuse, vol. $28, \mathrm{n}^{\circ} 1$, p. 41 .

Tradition axiomatisante, vol. $28, n^{\circ} 2$, p. 207

Traducteur camerounais, vol. $28, \mathrm{n}^{\circ} 1$, p. 20

Traducteur cultivé non điplômé, vol. $28, \mathrm{n}^{\circ} 3$, p. 302.

Traducteur de vingt-deux langues, vol. 28, $n^{\circ} 4$, p. 340 .

Traducteur d'organisations internationales, vol. 28 , $n^{\circ} 1$, p. 90

Traducteur fictif, vol. $28, n^{\circ} 4$, p. 334

Traducteur indépendant, vol. $28, \mathrm{n}^{\circ} 1$, p. 90.

Traducteur-lecteur, vol. $28, \mathrm{n}^{\circ} 2$, p. 207.

Traducteur-médiateur, vol. 28, no $^{\circ}$, p. 44.

Traducteur "occasionnel", vol. $28, n^{\circ} 1$, p. 18.

Traducteur-praticien, vol. $28, \mathrm{n}^{\circ} 2$, p. 207.

Traducteur salarié, vol. $28, \mathrm{n}^{0} 1$, p. 90.

Traductif, vol. $28, n^{\circ} 2$, p. 131 .

Traduction, vol. $28, \mathrm{n}^{\circ} 2$, p. 133

Traduction administrative, vol. $28, n^{\circ} 1$, p. 90 .

Traduction assistée par ordinateur, vol. $28, \mathrm{n}^{\circ} 1$, p. 92.

Traduction comme un subterfuge, vol. $28, n^{\circ} 4$, p. 334.

Traduction dans l'étude des langues, la, vol. 28 , no 3, p. 311 .

Traduction de contes, vol. $28, n^{\circ} 1$, p. 38 .

Traduction de l'anglais, vol. $28, n^{\circ} 4$, p. 411 .

Traduction de langue, vol. $28, n^{\circ} 2$, p. 149.

Traduction de poésie néerlandaise, vol. $28, n^{\circ} 4$, p. 339.

Traduction de texte, vol. $28, n^{\circ} 2$, p. 149

Traduction des chants populaires, vol. $28, n^{\circ} 4$ p. 339.

Traduction des didacticiels, vol. $28, n^{\circ} 1$, p. 94

Traduction du Coran, vol. 28, n $^{\circ} 4$, p. 340 .

Traduction du persan, vol. $28, n^{\circ} 4$, p. 339

Traduction du sanskrit, vol. $28, n^{\circ} 4$, p. 339 .
Traduction économique, vol. $28, n^{\circ} 1$, p. 90 .

Traduction en hébreu, vol. $28, \mathrm{n}^{\circ} 4$, p. 337 .

Traduction en swahili, vol. $28, n^{\circ} 4$, p. 337

Traduction en urdu, vol. $28, n^{\circ} 4$, p. 337.

Traduction et interprétation en Océanie, vol. 28 , $\mathrm{n}^{\circ} 1$, p. 17.

Traduction et linguistique, vol. $28, \mathrm{n}^{\circ} 2$, p. 130 .

Traduction et qualité de langue, vol. $28, \mathrm{n}^{0} 2$, p. 220.

Traduction, exercice traditionnel de la, vol. 28 , $\mathrm{n}^{\circ} 3$, p. 312 .

Traduction financière, vol. $28, \mathrm{n}^{\circ} 1$, p. 90

Traduction inégale, vol. $28, \mathrm{n}^{\circ} 4$, p. 353.

Traduction-introduction, vol. $28, \mathrm{n}^{\circ} 1, \mathrm{p} .45$.

Traduction juridique, vol. $28, \mathrm{n}^{\circ} 1$, p. 90 .

Traduction littéraire, vol. $28, \mathrm{n}^{\circ} 1$, p. 18 ; vol. 28 , $\mathrm{n}^{\circ} 1$, p. 90 ; vol. $28, \mathrm{n}^{\circ} 1$, p. 94.

Traduction mécanique, vol. $28, \mathrm{n}^{\circ} 2, \mathrm{p} .137$.

Traduction médicale, vol. $28, \mathrm{n}^{\circ} 1$, p. 90 .

Traduction moderne, précurseur de la, vol. 28 $\mathrm{n}^{\circ} 4$, p. 327

Traduction opératoire, vol. $28, n^{\circ} 1$, p. 90 .

Traduction ornée, vol. $28, \mathrm{n}^{\circ} 4$, p. 327 .

Traduction par des têtes couronnées, vol. $28, n^{\circ} 4$ p. 337.

Traduction pédagogique, vol. $28, \mathrm{n}^{\circ} 1, \mathrm{p} .94$.

Traduction, Pour une pédagogie de la, vol. 28 , $n^{\circ} 2$, p. 206.

Traduction pragmatique, vol. $28, \mathrm{n}^{\mathrm{o}} 1$, p. 90 vol. $28, n^{\circ} 2$, p. 133.

Traduction professionnelle, vol. $28, \mathrm{n}^{\circ} 1, \mathrm{p} .94$

Traduction religieuse, vol. $28, \mathrm{n}^{\circ} 4$, p. 342 .

Traduction, schéma détaillé de la, vol. $28, \mathrm{n}^{\circ} 2$, p. 206.

Traduction scolaire, vol. $28, n^{\circ} 1$, p. 18

Traduction simultanée, fondements théoriquesvol. $28, n^{\circ} 2$, p. 205.

Traduction technique, vol. $28, \mathrm{n}^{\circ} 1$, p. 90

Traduction-texte, vol. $28, \mathrm{n}^{\circ} 1$, p. 45 .

Traduction, théorie de la, vol. $28, \mathrm{n}^{\circ} 1$, p. 94

Traduction, unité de, vol. $28, \mathrm{n}^{\circ} 2$, p. 149.

Traduction utilitaire, vol. $28, \mathrm{n}^{\circ} 1$, p. 94 ; vol. 28 , $\mathrm{n}^{\circ} 2$, p. 133.

Traductionnel, vol. $28, n^{\circ} 2$, p. 131.

Traductionnel, processus, vol. $28, n^{\circ} 4$, p. 323 .

Traductionologie, vol. $28, n^{\circ} 2$, p. 131 .

Traductionologique, vol. $28, \mathrm{n}^{\circ} 2$, p. 131

Traductionologue, vol. $28, n^{\circ} 2$, p. 131

Traductologie, vol. $28, \mathrm{n}^{\circ} 1$, p. 94 ; vol. $28, \mathrm{n}^{\circ} 2$, p. 131.

Traduire, vol. $28, \mathrm{n}^{\circ} 1$, p. 35

Trait de scie, vol. 28, no $^{\circ}$, p. 194.

Trait de scie parallèle aux fibres, vol. $28, n^{\circ} 2$, p. 195.

Trait de scie perpendiculaire aux fibres, vol. 28 , $n^{\circ} 2$, p. 195.

Trait distinctif, vol. $28, \mathrm{n}^{\circ} 1$, p. 52 ; vol. $28, \mathrm{n}^{\circ} 3$, p. 280.

Trait phonémique, vol. $28, \mathrm{n}^{\circ} 2$, p. 145 .

Traitement acoustique, vol. $28, \mathrm{n}^{\circ} 4$, p. 406.

Transcrire, vol. $28, n^{\circ} 4$, p. 406

Transfert, opération de, vol. $28, n^{\circ} 4$, p. 324

Translateur, vol. $28, n^{\circ} 4$, p. 326 .

Translation, vol. $28, \mathrm{n}^{\circ} 1$, p. 45 .

Transmission du message, vol. $28, \mathrm{n}^{\circ} 3$, p. 237

Travail de terrain, vol. $28, n^{\circ} 4$, p. 406. 
Travail en cabine, vol. $28, n^{\circ} 3$, p. 237.

Travail, qualité du, vol. $28, n^{\circ} 3$, p. 239 Travaux, vol. $28, \mathrm{n}^{\circ} 1, \mathrm{p}$

Traverse, vol. 28 , no 2 , p. 193 ; vol. $28, \mathrm{n}^{\circ} 2$ p. 194 ; vol. $28, \mathrm{n}^{\circ} 2$, p. 196 .

Très gros plan, vol. $28, n^{\circ} 4$, p. 406 .

Tri-, vol. $28, n^{\circ} 2$, p. 170.

Triméthyl-aluminium, vol. $28, n^{\circ} 2$, p. 168 .

Triomphateur, vol. $28, n^{\circ} 2$, p. 160 .

Tris-, vol. $28, n^{\circ} 2$, p. 170.

Tronc tibio-péronier, vol. $28, n^{\circ} 4$, p. 384

Troncation, règle de, vol. $28, n^{\circ} 2$, p. 157 ; vol. 28 , $\mathrm{n}^{\circ} 2$, p. 163 .

Tronçonnage, vol. 28 , no 4 , p. 362

Trousse des traducteurs et des terminologues, vol. $28, \mathrm{n}^{\circ} 2$, p. 219.

Trouvaille poétique brésilienne, vol. $28, n^{\circ} 1, p$.

Tube en bande, vol. $28, n^{\circ} 3$, p. 291

Tube en nappe, vol. $28, n^{\circ} 3$, p. 291.

Type de vapeur, vol. $28, n^{\circ} 3$, p. 305 .

Typologie des néologismes français, vol. $28, n^{\circ} 3$, p. 299 .

UMA IDEIA TODA AZUL, vol. $28, n^{\circ} 1$, p. 35.

UNC, vol. 28 , no 1 , p. 21

Undéca-, vol. 28 , no 2 , p. 170

UNESCO, vol. 28, n $^{\circ} 1$, p. 19

Uniformité, vol. 28 , no 3 , p. 282

Union internationale de chimie pure et appliquée vol. $28, n^{\circ} 2$, p. 166.

Union Nationale Camerounaise, vol. 28, no 1 , p. 21.

Unité de traduction, vol. $28, n^{\circ} 2$, p. 149

Unité d'information, vol. $28, \mathrm{n}^{\circ} 3$, p. 241

Unité du discours, vol. $28, n^{\circ} 2$, p. 143.

UNITÉ, 1', vol. $28, \mathrm{n}^{\circ} 1$, p. 22.

Unité lexicale, vol. 28 , no 2 , p. 133

Unité sémantique, vol. 28, n० $^{\circ}$, p. 149.

Unité significative, vol. 28 , no 2 , p. 141

Universaux de syntaxe, vol. $28, n^{\circ} 1$, p. 51

Universaux du langage, vol. 28 , no 1, p. 45.

Université nationale de Papouasie, vol. $28, n^{\circ} 1$, p. 18.

Usance, rol. 28, n $^{\circ} 3$, p. 303.

Valeur affichée, vol. $28, n^{\circ} 4$, p. 366.

Valeur de consigne, vol. 28 , no 4 , p. 366

Valeur échantillonnée, vol. 28, n $^{\circ} 4$, p. 369.

Valeur instantanée, vol. $28, n^{\circ} 4$, p. 369.

Valeur motivée, vol. 28, no $^{\circ}$, p. 55 .

Valeur réelle, vol. $28, n^{\circ} 4$, p. 366

Valve, vol. $28, n^{\circ} 4$, p. 381 ; vol. $28, n^{\circ} 4$, p. 382.

Valvule, vol. $28, n^{\circ} 4$, p. 381 .

Valvule sigmoïde aortique, vol. $28, n^{\circ} 4$, p. 382.

Valvule sigmoïde pulmonaire, vol. 28, n $^{\circ} 4$, p. 382.

Valvule tricuspide, vol. $28, n^{\circ} 4$, p. 382 .

Vanuatu, vol. $28, n^{\circ} 1$, p. 17.

Variable, vol. $28, n^{\circ} 4$, p. 366

Variable de commande, vol. 28 , no 4 , p. 366

Variable principale, vol. $28, n^{\circ} 4$, p. 366

Variété régionale, vol. 28, no $^{\circ}$, p. 303.
Vent de pluie, vol. $28, n^{\circ} 3$, p. 294.

Vent pluvieux, vol. 28, no $^{\circ}$, p. 294.

Vento, vol. 28, n $^{\circ} 1$, p. 37

Ver, vol. $28, n^{\circ} 1$, p. 37

Vérification des pièces d'identité, vol. $28, n^{\circ} 1$, p. 23.

Vermouth de Chambéry, vol. $28, n^{\circ} 2$, p. 184

Verrouilleur de synchronisation, vol. $28, n^{\circ} 4$, p. 406 .

Version, vol. $28, \mathrm{n}^{\circ} 3$, p. 312 .

Vidange, vol. 28 , n० 3 , p. 291

Vide, vol. 28 , no 3 , p. 292.

Vide épistémologique, vol. $28, n^{\circ} 4$, p. 324

Vin, vol. $28, n^{\circ} 2$, p. 179 ; vol. $28, n^{\circ} 2$, p. 184.

Vin de liqueur, vol. $28, n^{\circ} 2, p .184$

Vin doux naturel, vol. $28, \mathrm{n}^{\circ} 2$, p. 184

Virtualité, vol. 28 , no 1, p. 48 .

Virtuème, vol. 28, no $^{\circ}$, p. 53

Vision du monde, vol. 28 , no 1 , p. 51 ; vol. 28 , $n^{\circ} 2$, p. 133 ; vol. 28 , n० 4 , p. 330 .

Visite officielle, en, vol. 28 , no 1, p. 23

Vivant, vol. 28, no 1, p. 53.

Vocabulaire brésilien, vol. $28, n^{\circ} 1$, p. 40

Vocabulaire contextuel du bâtiment, vol. $28, n^{\circ} 3$ p. 292

Vocabulaire de l'alcoolisme, vol. 28, n०2, p. 180.

Vodka, vol. 28, n $^{\circ} 2$, p. 184

Voie, vol. 28, n०2, p. 194.

Voie, donner de la, vol. $28, n^{\circ} 2$, p. 194.

Voix dans le champ, vol. $28, n^{\circ} 4$, p. 406.

Voix hors champ, vol. 28 , no 4 , p. 406

Volume massique, vol. $28, \mathrm{n}^{\circ} 3$, p. 299.

Wallis et Futuna, vol. 28, no 1 , p. 17.

Watt efficace, vol. $28, n^{\circ} 2$, p. 192.

Watt R.M.S., vol. 28, no 2, p. 192.

Whisky de grain, vol. 28, no 2, p. 185 .

Whisky de Malt, vol. $28, \mathrm{n}^{\circ} 2$, p. 185.

$\mathrm{X}$-eur, vol. $28, \mathrm{n}^{\circ} 2$, p. 157.

$\mathrm{X}$-iser, vol. $28, \mathrm{n}^{\circ} 2$, p. 158.

$X$-t-eur, vol. $28, n^{\circ} 2$, p. 157.

Xanthène, vol. $28, \mathrm{n}^{\circ} 2$, p. 170 .

Xat, vol. $28, n^{\circ} 2$, p. 161 .

Xculteur, vol. 28 , no 2 , p. 160.

Xérès, vol. 28 , n० 2 , p. 184.

-yne, vol. 28, n$^{\circ} 2$, p. 169.

Zone conceptuelle, vol. 28, n $^{\circ} 1$, p. 49.

Zone partagée, vol. $28, n^{\circ} 1$, p. 49 .

Zone sémantique, vol. $28, n^{\circ} 1$, p. 52. 


\section{Index anglais des mots et des sujets traités}

1,1-dichloro-1'-chloro-4-ethyl-1"chloro-7-propyldodecane, vol. 28 , no 2 , p. 171. 1,1-dichloro-4-(1-chloroethyl)-7-(1-chloropropyl) dodecane, vol. $28, \mathrm{n}^{\circ} 2$, p. 171.

11-nitro-2,5,8,10-tetraoxaundecane, vol. $28, n^{\circ} 2$, p. 169.

1,2,3,4-tetrahydronaphthalene, vol. $28, \mathrm{n}^{\circ} 2$, p. 168.

13-chloro-6,12-dioxa-3-thia-9-, vol. 28, n०2, p. 170 .

3-bromo-2-methyl-1,6-hexanediol, vol. $28, \mathrm{n}^{\circ} 2$, p. 167.

5,5-bis(1,1-dimethylpropyl)-2-methyldecane, vol. $28, n^{\circ} 2$, p. 171 .

5,5-bis-1',1'-dimethylpropyl-2-methyldecane, vol. $28, \mathrm{nl}^{\circ} 2$, p. 171.

5-hydroxy-2-pentanone, vol. $28, n^{\circ} 2$, p. 167.

"a " nomenclature, vol. $28, n^{\circ} 2$, p. 169

Abrupt language, vol. $28, n^{\circ} 1$, p. 101.

Absence of capitalization, vol. $28, \mathrm{n}^{\circ} 1$, p. 76 .

Absorber, vol. 28, no 3 , p. 288.

Absorptivity, vol. 28, n $^{\circ} 3$, p. 288.

Accuracy, vol. 28, no 3, p. 259.

Acetic anhydride, vol. 28 , no 2, p. 169.

Across the grain, vol. $28, n^{\circ} 2$, p. 195.

Act, vol. $28, n^{\circ} 4$, p. 375.4 , p. 373 .

Act of communication, vol. 28 , no 3 , p. 301.

Act of Parliament, vol. $28, n^{\circ} 4$, p. 370 .

Act of recognition, vol. $28, n^{\circ} 4$, p. 370 .

Action, vol. $28, n^{\circ} 2$, p. 173.

Action-eliciting function, vol. 28, no 3 , p. 253.

Active solar system, vol. $28, n^{\circ} 3$, p. 291

Actual value, vol. 28 , no 4 , p. 366 ; vol. 28 , no 4 , p. 369 .

Actuator, vol. 28 , no 4 , p. 367.

Adaptation, vol. 28, no $^{\circ}$, p. 255.

Additive nomenclature, vol. $28, \mathrm{n}^{\circ} 2$, p. 168

Adequacy, vol. $28, n^{\circ} 2$, p. 117.

Adequate translation, vol. 28, no 2, p. 117.

Adjourn, vol. $28, n^{\circ} 4$, p. 370 .

Adjournment, vol. $28, n^{\circ} 4$, p. 370 .

Adjournment debate, vol. 28 , no 4 , p. 370 .

Adjustable bow frame, vol. $28, n^{\circ} 4$, p. 365 .

Advanced lay-out, vol. $28, n^{\circ} 4$, p. 402.

Advertising man, vol. $28, n^{\circ} 4$, p. 404

African Native Literature, vol. $28, n^{\circ} 1$, p. 27.

Agglutinative language, vol. $28, \mathrm{n}^{\circ} 1$, p. 95 .
Air factor, vol. 28, no 3, p. 298.

Air heater, vol. 28, no 3 , p. 289.

Air print, vol. 28 , no 4 , p. 401 .

Air space, vol. 28, no 3, p. 292.

Air type collector, vol. 28, no $^{\circ}$, p. 289.

Alcoholic beverage, vol. 28 , no 2 , p. 178 .

Alcoholize, vol. 28, n०2, p. 183.

Alliterate doublet, vol. $28, \mathrm{n}^{\circ} 2$, p. 204.

Alliterates, vol. 28, no 2, p. 204.

Allowable, vol. 28, no 3, p. 259.

Along the grain, vol. $28, \mathrm{n}^{\mathrm{o}} 2$, p. 195.

A.M., vol. $28, n^{\circ} 2$, p. 192.

Ambiguity, vol. 28, n० 3, p. 308.

American teeth, vol. 28 , no 2 , p. 195.

Amplifier, vol. $28, n^{\circ} 2$, p. 186 ; vol. $28, n^{\circ} 2$, p. 191.

Amplitude modulation, vol. 28, no 2, p. 192.

Analog control, vol. $28, n^{\circ} 4$, p. 367.

Analog-to-digital converter, vol. 28, no 4, p. 369.

Analyzer, vol. $28, \mathrm{n}^{\circ} 2$, p. 212.

Anchor, vol. 28, no 3, p. 292.

Ancillary skills program, vol. $28, n^{\circ} 3$, p. 315

Angiotensiogen, vol. $28, n^{\circ} 4$, p. 379.

Anhydro-, vol. 28, n $^{\circ} 2$, p. 168.

Annotated videodisc, vol. $28, n^{\circ} 3$, p. 315 .

Annul, vol. 28 , no 4 , p. 375 .

Annulment, vol. 28 , no 4 , p. 375 .

Antihypertensive, vol. 28 , no 4, p. 378.

Antireflection coating, vol. $28, \mathrm{n}^{\circ} 3$, p. 290.

Anzeichen, vol. $28, n^{\circ} 3$, p. 300 .

Aortic arch syndrome, vol. $28, n^{\circ} 4$, p. 384 .

A pex organization, vol. $28, n^{\circ} 1$, p. 23.

A ppeals officer, vol. $28, n^{\circ} 1$, p. 23 .

Approach to Computer-Aided Translation, An, vol. $28, n^{\circ} 2$, p. 211 .

Appropriation act, vol. $28, n^{\circ} 4$, p. 370.

Aptitude testing, vol. $28, \mathrm{n}^{\circ} 3$, p. 316.

Arabic, vol. 28 , no 1 , p. 70 .

Arabic script, vol. 28 , no 1, p. 74.

Arabicization, vol. 28 , no 1, p. 71 .

Arabicization of foreign proper nouns, vol. 28 , $n^{\circ} 1$, p. 79 .

Arch of aorta, vol. $28, n^{\circ} 4$, p. 383

Area council, vol. $28, n^{\circ} 1$, p. 23.

Arterial blood pressure, vol. $28, n^{\circ} 4$, p. 378 .

Artificial intelligence, vol. $28, n^{\circ} 3$, p. 277 .

Artwork, vol. $28, n^{\circ} 4$, p. 401.

ASLIB, vol. $28, n^{\circ} 1$, p. 87.

Asociacion de Traductores Profesionales de México, vol. 28 , no 1 , p. 14

Assessing oral proficiency, vol. $28, n^{\circ} 3$, p. 316.

Assistant subdivisional officer, vol. 28, no 1, p. 23

Associate status, vol. $28, n^{\circ} 1$, p. 87.

Association of Indonesian Translators, vol. 28, $\mathrm{n}^{\circ} 1$, p. 14.

Association of Specialized Libraries, vol, $28, \mathrm{n}^{\circ} 1$, p. 87.

Athapaska, vol. $28, \mathrm{n}^{\circ} 2$, p. 153.

Atmosphere, vol. 28, no 3, p. 309.

Atrial systole, vol. $28, n^{\circ} 4$, p. 377 .

Atrio-ventricular node, vol. $28, n^{\circ} 4$, p. 381 .

Atrium, vol. $28, n^{\circ} 4$, p. 381 .

Audio, vol. 28, no 2, p. 191.

Audio equipment, vol. 28 , no 2 , p. 186 ; vol. 28 , $n^{\circ} 2$, p. 192.

Audio-video-split, vol. 28, no 4, p. 401 
Audiophile, vol. 28, n², p. 191.

Auditory area, vol. $28, n^{\circ} 2$, p. 191

Auditory orientation, vol. $28, n^{\circ} 2$, p. 192.

Auditory perspective, vol. 28, n०2, p. 191.

Auricle, vol. $28, n^{\circ} 4$, p. 381 .

Auriculoventricular, vol. $28, n^{\circ} 4$, p. 381 .

Auriculoventricular groove, vol. $28, n^{\circ} 4$, p. 381.

Authority, vol. $28, n^{\circ} 4$, p. 370 .

Author's intent, vol. $28, n^{\circ} 3$, p. 245.

Automatic controls, vol. $28, n^{\circ} 3$, p. 299.

Automatic correspondance, vol. 28, n० 3, p. 273.

Automatic Dictionary Compilation, vol. $28, n^{\circ} 2$, p. 211.

Automatic Language Processing, vol. 28, $\mathrm{n}^{\circ} 2$ p. 211 .

Automatic operator, vol. $28, n^{\circ} 3$, p. 299.

Automatic Translation, vol. $28, \mathrm{n}^{\circ} 2$, p. 211

Auxiliary heating, vol. $28, n^{\circ} 3$, p. 289.

Aviation system, vol. $28, \mathrm{n}^{\circ} 2$, p. 209.

Avoid repetition, vol. 28 , no 3 , p. 257

Azatetradecanoic acid, vol. 28 , no 2 , p. 170.

Back-transformation, vol. 28 , no 3, p. 253.

Back-up heating, vol. $28, n^{\circ} 3$, p. 289

Backed saw, vol. 28, n $^{\circ} 2$, p. 195.

Bag of words, vol. $28, n^{\circ} 1$, p. 49.

Bahasa Indonesia, vol. $28, n^{\circ} 1$, p. 11

Bahasa Malaysia, vol. $28, \mathrm{n}^{\circ} 1$, p. 11.

Balance control, vol. 28 , no 2, p. 191

Bandwidth, vol. 28, no 2, p. 191.

Bay of Yedo, vol. $28, \mathrm{n}^{\circ} 1$, p. 97.

BDÜ, vol. $28, n^{\circ} 1$, p. 87.

Bead, vol. $28, n^{\circ} 3$, p. 292.

Beer, vol. $28, \mathrm{n}^{\circ} 2$, p. 178

Benign, vol. $28, n^{\circ} 4$, p. 385

Benzofuran, vol. 28, n० 2, p. 170.

Benzothiophene, vol. 28 , no 2 , p. 170

Beta adrenergic blocking agent, vol. $28, \mathrm{n}^{\circ} 4$, p. 388 .

Beta adrenergic receptor blocking agent, vol. 28, $n^{\circ} 4$, p. 388 .

Beta blocker, vol. 28 , no 4 , p. 388 .

Beta blocking agent, vol. $28, n^{\circ} 4$, p. 388.

Bevel edge teeth, vol. $28, n^{\circ} 2$, p. 195.

Big close up, vol. 28, n० 4 , p. 406.

Bilingual paralegal, vol. 28, no 3, p. 315 .

Bilingual reporting, vol. $28, n^{\circ} 3$, p. 252

Bill, vol. $28, n^{0} 4$, p. 371 .

Billet saw, vol. $28, n^{\circ} 4$, p. 362

Binaural hearing, vol. 28 , no 2, p. 191.

Bird, vol. $28, \mathrm{n}^{\circ} 3$, p. 261.

Bit string, vol. 28, n $^{\circ} 4$, p. 369.

Blackbody, vol. $28, n^{\circ} 3$, p. 289.

Blade, vol. $28, n^{\circ} 2$, p. 196.

Blade holder pin, vol. 28 , no 4 , p. 362 .

Blind test, vol. $28, n^{\circ} 4$, p. 406.

Blood pressure, vol. 28 , no 4 , p. 378.

Board saw, vol. 28, n० 2, p. 198.

Bold translators, vol. 28, no 1, p. 100.

Book, vol. $28, n^{\circ} 1$, p. 78 .

Booster, vol. $28, n^{\circ} 4$, p. 405 .

Bow, vol. 28 , no 2 , p. 197.
Bow saw, vol. $28, n^{\circ} 2$, p. 196 ; vol. $28, n^{\circ} 2$, p. 197 ; vol. 28 , no 4 , p. 361 ; vol. $28, n^{\circ} 4$, p. 363 .

Bracket saw, vol. 28, n $^{\circ} 4$, p. 363 .

Brackets, vol. $28, n^{\circ} 1$, p. 76 .

Brain-storming, vol. $28, \mathrm{n}^{\circ} 4$, p. 404.

Brickwork, vol. 28, no 3, p. 292.

British bow saw, vol. 28, no $^{\circ}$, p. 361 .

Broad bladed, vol. $28, \mathrm{n}^{\circ} 2$, p. 197 ; vol. $28, \mathrm{n}^{\circ} 2$ p. 198.

Bromomethylhexanediol, vol. 28, no 2, p. 167.

Bruit, vol. $28, n^{\circ} 4$, p. 380 .

Buck saw, vol. $28, n^{\circ} 2$, p. 197 ; vol. $28, n^{\circ} 4$, p. 362 .

Bucksaw, vol. 28 , no 4 , p. 362 .

Buhl saw, vol. 28, n $^{\circ} 4$, p. 363 .

Bushi, vol. 28, no 1 , p. 96.

Bushido, vol. 28 , no 1 , p. 96

Bushman's saw, vol. 28 , no 4 , p. 363 .

Butt, vol. 28, n $^{\circ} 4$, p. 361 .

Butyrolactone, vol. $28, n^{\circ} 2$, p. 169.

Cableman, vol. $28, n^{\circ} 4$, p. 400

Calibrated, vol. 28 , no 4 , p. 366

Calibration, vol. $28, n^{\circ} 4$, p. 366.

Calling up of tenders, vol. $28, \mathrm{n}^{\circ} 4$, p. 375.

Cameraman, vol. $28, \mathrm{n}^{\circ} 4$, p. 400

Camp David, vol. 28 , no 1 , p. 78

Canada's far North, vol. 28 , no 2 , p. 153.

Canadian International Development Agency, vol. $28, n^{\circ} 1$, p. 11

Canadian North, vol. 28 , no 2 , p. 154

Cancel, vol. 28, no 4 , p. 375 .

Capillary break, vol. 28, no $^{\circ} 3$, p. 292.

Capitalization, vol. $28, n^{\circ} 1$, p. 76

Caprock, vol. $28, n^{\circ} 3$, p. 299.

Carbon chain, vol. $28, n^{\circ} 2$, p. 167

Cardiovascular system, vol. $28, n^{\circ} 4$, p. 379.

Cartridge, vol. 28, n० 2, p. 192.

Cast, vol. $28, n^{\circ} 4$, p. 401.

Casting, vol. $28, n^{\circ} 4$, p. 401.

Casting director, vol. $28, n^{\circ} 4$, p. 404

Catching, vol. $28, n^{\circ} 4$, p. 399.

Caustic potash solution, vol. $28, n^{\circ} 3$, p. 298.

Cavity wall, vol. $28, \mathrm{n}^{\circ} 3$, p. 292.

CCC roots, vol. $28, \mathrm{n}^{\circ} 1$, p. 58 ; vol $28, \mathrm{n}^{\circ} 1$, p. 60 .

Cellular structure, vol. $28, n^{\circ} 3$, p. 291

Centralized digital controller, vol. $28, n^{\circ} 4$, p. 369

Certified interpreter's work in german courts, vol. $28, n^{\circ} 3$, p. 315 .

Certiorari, vol. $28, n^{\circ} 4$, p. 371 .

Champion teeth, vol. 28, n $^{\circ} 2$, p. 195

Channel separation, vol. 28, no $^{\circ}$, p. 191.

Characteristic group, vol. $28, n^{\circ} 2$, p. 167.

Cheek, vol. $28, \mathrm{n}^{\circ} 2$, p. 196

Chief clerk, vol. $28, n^{\circ} 1$, p. 23.

Chief collector, vol. 28 , no 1, p. 23

Chief secretary, vol. $28, n^{\circ} 1$, p. 23.

Chieftancy dispute, vol. $28, n^{\circ} 1$, p. 23.

Chinese, vol. $28, n^{\circ} 1$, p. 95 .

Chinese Language and Research Centre, vol. 28 no 1, p. 13.

Church Missionary Society, vol. 28, no 1, p. 27. 
CIDA, vol. 28, no 1 , p. 11.

Cider, vol. $28, \mathrm{n}^{\circ} 2$, p. 178

Circulation pump, vol. 28, no $^{\circ}$, p. 290.

Circulator, vol. $28, n^{\circ} 3$, p. 290.

Circulatory system, vol. $28, n^{\circ} 4$, p. 379 .

CISAC/CIAL, vol. $28, \mathrm{n}^{\circ} 1$, p. 86 .

Citation, vol. $28, n^{\circ} 3$, p. 271.

City, vol. $28, n^{\circ} 1$, p. 78.

Cladding, vol. $28, \mathrm{n}^{\circ} 3$, p. 292.

Classic for children, vol. $28, \mathrm{n}^{\circ} 3$, p. 255.

Clause, vol. $28, n^{\circ} 4$, p. 371 ; vol. $28, n^{\circ} 4$, p. 375 .

Clean-list, vol. $28, n^{\circ} 4$, p. 404

Close up, vol. $28, n^{\circ} 4$, p. 402 ; vol. $28, n^{\circ} 4$, p. 403.

Clutch of eggs, vol. $28, n^{\circ} 3$, p. 261

Co-writing, vol. $28, n^{\circ} 1$, p. 108.

Codebook, vol. $28, n^{\circ} 3$, p. 271.

Cognitive dissonance, vol. $28, \mathrm{n}^{\circ} 1$, p. 101.

Coherence, vol. $28, n^{\circ} 3$, p. 268.

Collection, vol. $28, n^{\circ} 3$, p. 289.

Collector tilt, vol. $28, n^{\circ} 3$, p. 289.

Collector tilt angle, vol. $28, \mathrm{n}^{\circ} 3$, p. 289.

Colo(u)ration, vol. $28, \mathrm{n}^{\circ} 2$, p. 191.

Comic text, vol. 28, no $^{\circ}$, p. 301

Command variable, vol. $28, n^{\circ} 4$, p. 366

Commission, vol. $28, n^{\circ} 4$, p. 371

Commissioner, vol. 28, no 4 , p. 372.

Committee, vol. $28, n^{\circ} 4$, p. 372.

Common Market, vol. 28, $n^{\circ} 1$, p. 8.

Commune, vol. $28, n^{\circ} 4$, p. 376.

Communication, vol. $28, n^{\circ} 3$, p. 270.

Communication in Translated Texts : A Semiotic Approach, vol. $28, n^{\circ} 3$, p. 301 .

Communicative competence, vol. $28, n^{\circ} 3$, p. 306

Community development officer, vol. $28, \mathrm{n}^{\circ} 1$, p. 23.

Compact disc, vol. $28, n^{\circ} 4$, p. 401.

Compactness, vol. $28, n^{\circ} 1$, p. 58 .

Comparator, vol. $28, n^{0} 4$, p. 367

Comparing element, vol. $28, n^{\circ} 4$, p. 367.

Compass sawing, vol. $28, n^{\circ} 4$, p. 358 .

Compass wood, vol. $28, n^{\circ} 4$, p. 358 .

Compilation of word elements, vol. $28, \mathrm{n}^{\circ} 2$, p. 222.

Complexity of sentences, vol. $28, n^{\circ} 4$, p. 347 .

Compound word, vol. $28, \mathrm{n}^{\circ} 2$, p. 205.

Computational linguistic, vol. $28, \mathrm{n}^{\circ} 2$, p. 210

Computer, vol. $28, n^{\circ} 3$, p. 315.

Computer Aided Translation, vol. $28, n^{\circ} 2$, p. 211.

Computer-assisted instruction, vol. $28, n^{\circ} 3$, p. 315.

Computer assisted translation, vol. $28, n^{\circ} 3$, p. 315 .

Computer for compiling special dictionary, vol. $28, \mathrm{n}^{\circ} 2$, p. 212.

Computer-implemented text processing, vol. 28, $n^{\circ} 2$, p. 211.

Concentrating collector, vol. $28, n^{\circ} 3$, p. 289.

Concept, vol. 28 , no 2 , p. 222 ; vol. $28, n^{\circ} 3$, p. 300

Concordance, vol. $28, \mathrm{n}^{\circ} 2$, p. 212

Condensation gutter, vol. $28, \mathrm{n}^{\mathrm{o}} 3$, p. 292.

Conditions of the contract, vol. $28, n^{\circ} 4$, p. 375

Conduce, vol. $28, \mathrm{n}^{\circ} 2$, p. 164

Conduct, vol. $28, \mathrm{n}^{\circ} 2$, p. 164 .

Conjunctive nomenclature, vol. $28, n^{\circ} 2$, p. 169

Consecutive interpretation, vol. $28, n^{\circ} 3$, p. 315 .
Consist in, vol. 28, no 3 , p. 272.

Consist of, vol. 28, no $^{\circ}$, p. 272 .

Content, vol. $28, \mathrm{n}^{\circ} 3$, p. 270.

Contextless sentence, vol. $28, n^{\circ} 3$, p. 273.

Contextual clue, vol. 28 , no 3 , p. 309.

Contextual cues, vol. 28, no $^{\circ}$, p. 316 .

Continent, vol. $28, n^{\circ} 1$, p. 78 ; vol. $28, n^{\circ} 1$, p. 78.

Continental frame saw, vol. $28, \mathrm{n}^{\circ} 2$, p. 196 ;

vol. $28, \mathrm{n}^{\circ} 2$, p. 197 ; vol. $28, \mathrm{n}^{\circ} 4$, p. 358 ;

vol. $28, \mathrm{n}^{\circ} 4$, p. 359 ; vol. $28, \mathrm{n}^{\circ} 4$, p. 360 ; vol. $28, n^{\circ} 4$, p. 361 .

Continuity/discontinuity focus, vol. $28, \mathrm{n}^{\circ} 3$, p. 308.

Contraction, vol. $28, n^{\circ} 3$, p. 292

Control hypertension, vol. $28, n^{\circ} 4$, p. 386.

Control signal, vol. $28, n^{\circ} 4$, p. 369.

Control track, vol. $28, n^{\circ} 4$, p. 403 .

Control unit, vol. $28, n^{\circ} 4$, p. 367 .

Control with fixed set point, vol. 28 , no 4 , p. 366

Controlled variable, vol. $28, n^{\circ} 4$, p. 366 .

Controller, vol. 28 , no 4 , p. 367.

Conventional tonearm, vol. $28, \mathrm{n}^{\circ} 2$, p. 191

Conversion efficiency, vol. $28, n^{\circ} 3$, p. 290.

Conversion factor, vol. $28, \mathrm{n}^{\circ} 3$, p. 290.

Cooper's frame saw, vol. $28, n^{\circ} 2$, p. 197 ; vol. 28 $n^{\circ} 4$, p. 362.

Cooper's head saw, vol. $28, \mathrm{n}^{\circ} 4$, p. 362 .

Coping saw, vol. $28, n^{\circ} 2$, p. 197 ; vol. $28, n^{\circ} 4$, p. 364.

Copy platform, vol. $28, n^{\circ} 4$, p. 400

Copy testing, vol. $28, n^{\circ} 4$, p. 406

Correct usage, vol. $28, \mathrm{n}^{\circ} 3$, p. 272.

Correct usage model, vol. $28, n^{0} 3$, p. 272

Correcting signal, vol. $28, n^{\circ} 4$, p. 367.

Correspondance, vol. 28, n० 3, p. 263.

Country, vol. $28, n^{\circ} 1$, p. 78 .

Course content, vol. $28, n^{\circ} 3$, p. 307

Course syllabus, vol. $28, \mathrm{n}^{\circ} 3$, p. 315 .

Courses, vol. $28, n^{\circ} 3$, p. 293.

Court interpreting, vol. $28, n^{\circ} 3$, p. 315

Cover, vol. 28, no $^{\circ}$, p. 289.

Cover plate, vol. $28, n^{\circ} 3$, p. 289

Cracking, vol. $28, \mathrm{n}^{\circ} 3$, p. 293.

Craftman, vol. $28, n^{\circ} 1$, p. 96.

Creativity, vol. $28, n^{\circ} 3$, p. 255 ; vol. $28, n^{\circ} 3$, p. 272.

Crest power, vol. 28, no 2 , p. 192.

Criminal procedure, vol. $28, n^{0} 3$, p. 315.

Cross fading, vol. 28 , no 4 , p. 402 .

Crosscut saw, vol. $28, n^{\circ} 4$, p. 359

Cryptography, vol. $28, n^{\circ} 3$, p. 276

Cultural-equivalence approach, vol. $28, n^{\circ} 3$, p. 245.

Culture, vol. $28, \mathrm{n}^{\circ} 3$, p. 245 ; vol. $28, \mathrm{n}^{\circ} 3$, p. 315.

Cup, vol. $28, n^{\circ} 4$, p. 382.

Current affairs, vol. $28, n^{\circ} 4$, p. 375 .

Curriculum, vol. 28 , no 3 , p. 315

Curriculum change, vol. 28, no $^{\circ}$, p. 315 .

Curriculum design, vol. 28, no 3 , p. 315 .

Curtain wall, vol. $28, n^{\circ} 3$, p. 293.

Curved sawing, vol. $28, n^{\circ} 4$, p. 358.

Cut, vol. $28, n^{\circ} 4$, p. 404 ; vol. 28 , no 4 , p. 405.

Cut off, vol. $28, n^{\circ} 4$, p. 403.

Cut up, vol. 28, n $^{\circ} 4$, p. 359.

Cyclohexanecarbodithionic acid, vol. $28, n^{\circ} 2$, p. 169. 
Daimos, vol. $28, \mathrm{n}^{\circ} 1$, p. 98.

Dangling participle, vol. $28, \mathrm{n}^{\circ} 3$, p. 272.

D.A.R., vol. 28 , no 4 , p. 406 .

Data, vol. $28, n^{\circ} 3$, p. 272

Datums, vol. $28, n^{\circ} 3$, p. 272.

Day, vol. $28, \mathrm{n}^{\circ} 1$, p. 78

Day after recall, vol. $28, n^{\circ} 4$, p. 406.

DB, vol. $28, \mathrm{n}^{\circ} 2$, p. 191.

DDC, vol. $28, \mathrm{n}^{\circ} 4$, p. 367.

Decibel, vol. $28, n^{\circ} 2$, p. 191.

Decomposition of a source text, vol. $28, n^{\circ} 2$, p. 117.

Decomposition of source message, vol. $28, n^{\circ} 3$, p. 301.

Deduce, vol. 28, n० 2, p. 159.

Deduction, vol. $28, \mathrm{n}^{\circ} 2$, p. 159

Deep throat coping saw, vol. 28, no 4, p. 363.

Defect, vol. $28, \mathrm{n}^{\circ} 3$, p. 276

Defining translation, vol. $28, n^{\circ} 3$, p. 301.

Definition, vol. $28, n^{\circ} 3$, p. 271 ; vol. $28, n^{\circ} 3$, p. 315 .

Definition of translation, vol. $28, n^{\circ} 2$, p. 117.

Deflection, vol. $28, \mathbf{n}^{\circ} 3$, p. 293 .

Degree-day, vol. 28, no $^{\circ} 3$, p. 290.

Degree of equivalence, vol. $28, \mathrm{n}^{\circ} 3$, p. 266.

Dehydro-, vol. $28, n^{\circ} 2$, p. 168

Delay, vol. $28, n^{\circ} 4$, p. 367 .

Dene, vol. $28, \mathrm{n}^{\circ} 2$, p. 153.

Deoxy-, vol. 28, n $^{\circ} 2$, p. 168.

Deoxyribose, vol. $28, n^{\circ} 2$, p. 169 .

Departemental commission, vol. $28, n^{\circ} 4$, p. 376.

Department, vol. $28, n^{\circ} 3$, p. 260.

Department of English, vol. 28, no 1, p. 13.

Design, vol. $28, n^{\circ} 4$, p. 405.

Designer, vol. $28, n^{\circ} 4$, p. 405.

Deviation from maximally adequate tranlation, vol. $28, n^{\circ} 2$, p. 129.

Diastole, vol. $28, n^{\circ} 4$, p. 377

Dictionary, vol. $28, \mathrm{n}^{\circ} 3$, p. 270.

Dictionary article, vol. $28, n^{\circ} 3$, p. 271.

Dictionary model, vol. $28, \mathrm{n}^{\mathrm{o}} 3$, p. 271 .

Diethyl ether, vol. $28, n^{\circ} 2$, p. 168 .

Diffusion, vol. $28, n^{\circ} 1$, p. 9.

Diffusion of new technology, vol. $28, n^{\circ} 1$, p. 9 .

Digital control, vol. $28, n^{\circ} 4$, p. 367

Digital-to-analog control loop, vol. $28, n^{\circ} 4$, p. 369.

Digital-to-analog converter, vol. $28, n^{\circ} 4$, p. 369.

Digitizer, vol. $28, n^{\circ} 4$, p. 369.

Digression, vol. $28, \mathrm{n}^{\circ} 3$, p. 308.

DIN 2331, vol. $28, n^{\circ} 2$, p. 222.

Diploma in Scientific Translation, vol. $28, \mathrm{n}^{\circ} 1$, p. 7 .

Direct digital control, vol. $28, n^{\circ} 4$, p. 367 .

Direct solar system, vol. $28, \mathrm{n}^{\circ} 3$, p. 291.

Directional hearing, vol. $28, n^{\circ} 2$, p. 192.

Dirt road, vol. $28, \mathrm{n}^{\circ} 1$, p. 23.

Dispatch clerk, vol. 28, no 1, p. 23.

Display, vol. $28, n^{\circ} 4$, p. 400 .

Displayed value, vol. $28, n^{\circ} 4$, p. 366 .

Distillation, vol. $28, \mathrm{n}^{\circ} 2$, p. 178

Distilled spirits, vol. $28, \mathrm{n}^{\circ} 2$, p. 178

Distinctive feature, vol. $28, \mathrm{n}^{\circ} 2$, p. 120

Distortion ratio, vol. $28, \mathrm{n}^{\circ} 2$, p. 192.

Disturbance, vol. $28, n^{\circ} 4$, p. 366.

Disturbance variable, vol. $28, n^{\circ} 4$, p. 366 .
Double inverted commas, vol. $28, \mathrm{n}^{\circ} 1$, p. 76.

Double saw, vol. $28, \mathrm{n}^{\circ} 2$, p. 196.

Doublet, vol. $28, \mathrm{n}^{\circ} 2$, p. 204

DP 704, vol. $28, \mathrm{n}^{\circ} 2$, p. 222.

DP 860 , vol. 28, n $^{\circ} 2$, p. 222

Drafting stage, vol. $28, n^{\circ} 3$, p. 266

Drain down, vol. 28, no 3, p. 291.

Drip, vol. $28, \mathrm{n}^{\circ} 3$, p. 293.

Drive-in cinema, vol. 28 , no 4 , p. 400 .

Drop out, vol. $28, n^{\circ} 4$, p. 387 ; vol. $28, n^{\circ} 4$, p. 403.

Dropout, vol. $28, n^{\circ} 4$, p. 387 .

Drumwall, vol. $28, n^{\circ} 3$, p. 290.

Dubbing, vol. $28, \mathrm{n}^{\circ} 1, \mathrm{p} .110$.

Dupe, vol. $28, \mathrm{n}^{\circ} 4$, p. 402.

Dust, vol. 28, n० 2, p. 194.

Dynamic equivalence, vol. $28, \mathrm{n}^{\circ} 3$, p. 252.

Easy-chair, vol. $28, n^{\circ} 3$, p. 310.

Editing stage, vol. $28, n^{\circ} 3$, p. 267.

Edo, vol. 28 , no 1 , p. 28.

EEC, vol. 28, no $^{\circ} 1$, p. 8.

Effect, vol. 28, no 3, p. 270.

Effect on the reader, vol. $28, n^{\circ} 3$, p. 245.

Efflorescence, vol. $28, n^{\circ} 3$, p. 293.

Efik, vol. $28, n^{\circ} 1$, p. 27.

Eigenfrequency, vol. $28, \mathrm{n}^{\circ} 2$, p. 191 .

Electronic medium, vol. $28, \mathrm{n}^{\circ} 4$, p. 344 .

Electronic news gathering, vol. $28, n^{\circ} 4$, p. 402.

Electronic translation, vol. $28, n^{\circ} 1$, p. 110

Emissivity, vol. 28, no 3, p. 290.

Emphasis, vol. 28, no 3, p. 308.

Emphatic partner, vol. $28, n^{\circ} 1$, p. 74.

Enabling act, vol. $28, n^{\circ} 4$, p. 370

Encyclopedia Britannica translated into Chinese, vol. $28, n^{\circ} 1$, p. 14.

-ene, vol. 28, no 2, p. 169.

English grammar, vol. $28, n^{\circ} 3$, p. 306.

English Language Institute, vol. 28, no 1, p. 13.

English proper nouns into Arabic, vol. 28, no 1 , p. 71.

English scientific writing, vol. $28, n^{\circ} 3$, p. 261.

Environment, vol. 28, no 4, p. 366 .

Equalizer, vol. $28, n^{\circ} 4$, p. 402.

Equilibrium temperature, vol. $28, n^{\circ} 3$, p. 291.

Equivalence, vol. $28, n^{\circ} 2$, p. 117 ; vol. $28, n^{\circ} 3$, p. 245.

Equivalence-seeker, vol. 28, no 3, p. 249.

Era of Bold Translations, vol. $28, \mathrm{n}^{\circ} 1$, p. 100.

Error, vol. $28, \mathrm{n}^{\circ} 3$, p. 307 ; vol. $28, \mathrm{n}^{\circ} 4$, p. 367.

Error-based teaching, vol. 28, no 3 , p. 307.

Eskimo, vol. 28, no 2, p. 153.

Establishment of invariant, vol. 28, no 3, p. 301.

Ethyl alcohol, vol. 28, $\mathrm{n}^{\circ} 2$, p. 178.

Ethyl methyl ketone, vol. 28, no 2, p. 168.

Ethyl oxide, vol. $28, n^{\circ} 2$, p. 168.

Ethylene dichloride, vol. $28, \mathrm{n}^{\circ} 2$, p. 168

Ethylene oxide, vol. $28, n^{\circ} 2$, p. 168.

EURATOM, vol. $28, n^{\circ} 1$, p. 7

Europe, vol. $28, n^{\circ} 1$, p. 78 .

European Economic Community, vol. 28, $\mathrm{n}^{\circ} 1$, p. 8.

Eutectic plate, vol. 28, n$^{\circ} 3$, p. 290

Eutectic salt, vol. $28, \mathrm{n}^{\circ} 3$, p. 290 
Evacuation roads, vol. $28, n^{\circ} 1$, p. 23.

Evaluation of student performance, vol. $28, \mathrm{n}^{\circ} 3$, p. 316.

Evaluative analysis, vol. $28, n^{\circ} 3$, p. 251.

Excess air factor, vol. $28, n^{\circ} 3$, p. 298

Excess air number, vol. $28, n^{\circ} 3$, p. 298.

Excess air ratio, vol. 28, no 3, p. 298.

Excess verbiage, vol. $28, \mathrm{n}^{\circ} 3$, p. 261.

Expansion, vol. $28, n^{\circ} 3$, p. 293.

Expecting, vol. 28, no 3, p. 269.

Expressive function, vol. 28, no 3, p. 253.

Extended paraphrase, vol. 28, no 3, p. 263.

Extratextual material, vol. 28, no 3, p. 266.

Face-to-face speech, vol. $28, n^{\circ} 3$, p. 245.

Facsimile, vol. 28, n $^{\circ} 3$, p. 267.

Faculty of Languages, vol. 28, no 1, p. 10.

Fade in, vol. $28, n^{\circ} 4$, p. 403

Fade out, vol. $28, \mathrm{n}^{\circ} 4$, p. 402

Fader, vol. $28, n^{\circ} 4$, p. 402.

Fading, vol. $28, n^{\circ} 4$, p. 402.

Falcon, vol. $28, n^{\circ} 3$, p. 254.

False doublet, vol. $28, \mathrm{n}^{\circ} 2$, p. 205.

Family resemblance, vol. $28, \mathrm{n}^{\circ} 3$, p. 301.

Farmer, vol. 28, no 1, p. 96.

Feature, vol. 28, no 2, p. 117

Feedback control, vol. $28, n^{\circ} 4$, p. 366.

Feedforword control, vol. $28, n^{\circ} 4$, p. 366 .

Festival, vol. $28, \mathrm{n}^{\circ} 1$, p. 78 .

Fictitious text, vol. $28, n^{\mathrm{o}} 3$, p. 300 .

FID-Congress in Hong Kong, vol. 28, $\mathrm{n}^{\circ} 2$, p. 222.

Field work, vol. $28, n^{\circ} 4$, p. 406

Filing system, vol. $28, n^{\circ} 4$, p. 345.

Final endings on verbs, vol. $28, n^{\circ} 1$, p. 101.

Find, vol. 28, n० 3, p. 254.

Finding equivalent, vol. $28, \mathrm{n}^{\circ} 3$, p. 253.

Fine teeth, vol. $28, n^{\circ} 4$, p. 364 .

Firewood saw, vol. 28, no 4, p. 362.

Firing rate, vol. $28, \mathrm{n}^{\circ} 3$, p. 299.

First heart sound, vol. $28, n^{\circ} 4$, p. 380 .

FIT, vol. $28, \mathrm{n}^{\circ} 1$, p. 14 ; vol. $28, \mathrm{n}^{\circ} 1$, p. 86.

Fixed blade, vol. $28, n^{\circ} 4$, p. 358.

Fixed pivot arm, vol. $28, n^{\circ} 2$, p. 191.

Flash-back, vol. $28, n^{\circ} 4$, p. 404.

Flat-plate collector, vol. $28, n^{\circ} 3$, p. 289.

Fleam teeth, vol. $28, n^{\circ} 2$, p. 195 ; vol. $28, n^{\circ} 4$, p. 358.

Floppy disk, vol. 28, no $^{\circ} 4$, p. 344.

F.M., vol. 28, n² 2, p. 192.

Focusing collector, vol. $28, n^{\circ} 3$, p. 289

Folder, vol. $28, n^{\circ} 4$, p. 403.

Folder-test, vol. 28, no 4, p. 406

Follow-up control, vol. $28, n^{\circ} 4$, p. 366

Footnote, vol. 28 , no 3 , p. 259

Foreign language instruction, vol. $28, n^{\circ} 3$, p. 315 .

Foreign language school, vol. $28, \mathrm{n}^{\circ} 1$, p. 97.

Forestall, to, vol. $28, n^{\circ} 3$, p. 269.

Forester's saw, vol. $28, n^{\circ} 4$, p. 363.

Form, vol. 28 , no 2 , p. 164 ; vol. $28, n^{\circ} 3$, p. 315 .

Formal equivalent, vol. 28 , no 3, p. 253

Formalism, vol. $28, \mathrm{n}^{\circ} 3$, p. 255

Formate, vol. $28, \mathrm{n}^{\circ} 2$, p. 164.

Frame, vol. $28, \mathrm{n}^{\circ} 2$, p. 196.
Frame saw, vol. $28, n^{\circ} 2$, p. 195 ; vol. $28, n^{\circ} 2$, p. 196.

Framed bow saw, vol. 28 , no 4 , p. 361 .

Framed pit saw, vol. $28, n^{\circ} 2$, p. 197 ; vol. 28 , $\mathrm{n}^{\circ} 2$, p. 198

Framed-saw, vol. 28, no 2, p. 196.

French aperitif, vol. $28, \mathrm{n}^{\mathrm{0}} 3$, p. 309

French scientific writing, vol. 28, no $^{\circ} 3$, p. 261.

Frequency, vol. $28, \mathrm{n}^{\circ} 2$, p. $191 ;$ vol. $28, \mathrm{n}^{\circ} 2$, p. 192.

Frequency modulation, vol. $28, \mathrm{n}^{\circ} 2$, p. 192.

Fret saw, vol. $28, n^{\circ} 2$, p. 197 ; vol. $28, n^{\circ} 4$, p. 363.

Fretsaw, vol. $28, n^{\circ} 4$, p. 363

Full membership, vol. $28, \mathrm{n}^{\circ} 1$, p. 87.

Function, vol. $28, \mathrm{n}^{\circ} 2$, p. 167 ; vol. $28, \mathrm{n}^{\circ} 3$, p. 245 ; vol. 28 , no 3 , p. 315 .

Functional class name, vol. $28, n^{\circ} 2$, p. 168.

Functional group, vol. 28, $\mathrm{n}^{\circ} 2$, p. 167

Fundamental, vol. 28, no 2, p. 192.

Fundamental harmonic, vol. 28, n $^{\circ} 2$, p. 192

Fundamental tone, vol. $28, n^{\circ} 2$, p. 192

Fundus of gall bladder, vol. $28, \mathrm{n}^{\circ} 4$, p. 388 .

Fundus uteri, vol. 28 , no 4 , p. 388 .

Fundus ventriculi, vol. $28, n^{\circ} 4$, p. 388

Fused heterocyclic system, vol. $28, n^{\circ} 2$, p. 170.

Fusion name, vol. $28, n^{\circ} 2$, p. 170.

Gallicism, vol. $28, n^{\circ} 3$, p. 261.

Gas-expansion thermometer, vol. 28, no 3, p. 298.

Gen-lock, vol. $28, n^{\circ} 4$, p. 406

Geographical names, vol. $28, \mathrm{n}^{\circ} 1$, p. 78

German bow saw, vol. 28 , no 4 , p. 360 .

German Terminology of Data Processing, vol. 28, $n^{\circ} 2$, p. 211.

Glosing, vol. $28, n^{\circ} 3$, p. 273.

Goal-oriented phenomenon, vol. $28, \mathrm{n}^{0} 3$, p. 301.

Good writing, vol. $28, n^{\circ} 3$, p. 270.

Grain, vol. $28, n^{\circ} 2$, p. 195.

Grammatical item, vol. $28, n^{\circ} 3$, p. 307.

Grammatical number, vol. $28, \mathrm{n}^{\circ} 3$, p. 272

Grapheme, vol. 28, no 2, p. 120.

Graphic substance, vol. $28, n^{\circ} 2$, p. 120.

Graphical representation, vol. $28, n^{\circ} 2$, p. 122.

Graphological problem, vol. $28, \mathrm{n}^{\mathrm{o}} 1, \mathrm{p} .73$

Graphological translation, vol. $28, \mathrm{n}^{\circ} 1$, p. 70 ; vol. $28, \mathrm{n}^{\circ} 2, \mathrm{p} .120$

Grass-widow, vol. 28, no 1, p. 50.

Gravel bed storage, vol. $28, n^{\circ} 3$, p. 291.

Greenhouse effect, vol. $28, n^{\circ} 3$, p. 290.

Grog, vol. 28, no 2, p. 178 .

Gullet, vol. 28, no 2, p. 195.

Hack saw, vol. $28, n^{\circ} 2$, p. 197 ; vol. $28, n^{\circ} 4$, p. 365 .

Handled saw, vol. 28, no 2, p. 195.

Handling over notes, vol. $28, \mathrm{n}^{\circ} 1$, p. 23.

Harmonic, vol. $28, \mathrm{n}^{\circ} 2, \mathrm{p} .191$.

Hausa, vol. $28, n^{\circ} 1$, p. 27

Hearing loss, vol. $28, n^{\circ} 2$, p. 191

Heat input, vol. $28, n^{\circ} 3$, p. 299

Heat pipe, vol. $28, n^{\circ} 3$, p. 289. 
Hebrew, vol. $28, \mathrm{n}^{\circ} 1$, p. 57 ; vol. $28, \mathrm{n}^{\circ} 1$, p. 58. Heliothermal conversion, vol. $28, \mathrm{n}^{\circ} 3$, p. 289. Hertz, vol. 28, $\mathrm{n}^{\circ}$ 2, p. 191.

Hetero-atom, vol. $28, n^{\circ} 2$, p. 167

Hi-fi, vol. 28, no $^{\circ}$, p. 191 ; vol. 28, n $^{\circ} 2$, p. 191. Hierarchies of relevancy, vol. $28, \mathrm{n}^{\circ} 2$, p. 117 .

Hifi, vol. $28, n^{\circ} 2$, p. 191.

High-fidelity, vol. $28, \mathrm{n}^{\circ} 2$, p. 191

High technology, vol. 28 , no 1 , p. 9.

Himpunan Penterjemah Indonesia, vol. $28, n^{\circ} 1$, p. 14

Hold the tension, vol. $28, n^{\circ} 4$, p. 364

Honeycomb, vol. 28 , no 3, p. 291.

Honeycomb cellular structure, vol. $28, n^{\circ} 3$, p. 291.

Honeycomb structure, vol. 28 , n० 3, p. 291.

Honorable members, vol. $28, n^{\circ} 1$, p. 23.

Hopefully, vol. $28, n^{0} 3$, p. 272.

House of chiefs, vol. $28, n^{\circ} 1$, p. 23

Hybrid bill, vol. $28, n^{\circ} 4$, p. 371 .

Hydroxypentanone, vol. 28, $\mathrm{n}^{\circ}$ 2, p. 167.

Hypertension, vol. $28, n^{\circ} 4$, p. 378.

Hypertensive, vol. $28, n^{\circ} 4$, p. 378 .

$\mathrm{Hz}$, vol. $28, \mathrm{n}^{\circ} 2$, p. 191

IBM-Deutschland, vol. $28, \mathrm{n}^{\circ} 2$, p. 211

Ice-crystal theory, vol. $28, \mathrm{n}^{\circ} 3, \mathrm{p} .255$

Identification campaign, vol. $28, \mathrm{n}^{\circ} 1$, p. 23.

Idoma, vol. $28, \mathrm{n}^{\circ} 1$, p. 27.

Igbo, vol. $28, \mathrm{n}^{0} 1$, p. 27

ljaw, vol. $28, n^{\circ} 1$, p. 27.

Imam, vol. $28, n^{\circ} 1, p .9$

Immediate Constituent Analysis, vol. 28, $\mathrm{n}^{\circ} 2$, p. 151.

Imperative function, vol. $28, n^{\circ} 3$, p. 253

Impersonal construction, vol. $28, \mathrm{n}^{\circ} 3$, p. 308.

Implantation, vol. $28, n^{\circ} 1$, p. 9 .

Incomplete transfer, vol. $28, n^{\circ} 3$, p. 259.

Index Translationum, vol. $28, \mathrm{n}^{\circ} 1$, p. 102

Indian, vol. $28, n^{\circ} 2$, p. 153

Indian National Scientific Documentation Centre vol. $28, n^{\circ} 1$, p. 7.

Indian Scientific Translators Association, vol. 28, no 1, p. 7.

Indirect solar system, vol. $28, n^{\circ} 3$, p. 291.

Individual analog control loop, vol. $28, n^{\circ} 4$, p. 369 .

Indonesian, vol. $28, \mathrm{n}^{\circ} 1$, p. 11 .

Infiltration, vol. $28, n^{\circ} 3$, p. 293.

Information, vol. $28, \mathrm{n}^{\circ} 3$, p. 270

Information-moving, vol. $28, n^{\circ} 3$, p. 270.

Informative function, vol. $28, n^{\circ} 3$, p. 253

Infoterm Newletter 27 , vol. $28, n^{\circ} 2$, p. 221

Initial reference, vol. $28, n^{\circ} 3$, p. 308.

Inlaying saw, vol. $28, n^{\circ} 4$, p. 363.

Input, vol. $28, n^{\circ} 4$, p. 366 .

INSDOC, vol. 28 , no 1, p. 7 .

Instataneous value, vol. $28, \mathrm{n}^{\circ} 4$, p. 369 .

Institude of African Studies, vol. $28, \mathrm{n}^{\circ} 1$, p. 33

Institute of Linguists, vol. $28, n^{\circ} 1$, p. 86 .

Institute of Pacific Studies, vol. $28, n^{\circ} 1$, p. 18.

Institute of Translation and Interpreting, vol. 28, $\mathrm{n}^{\mathrm{o}} 1, \mathrm{p} .12$

Institute Voor Vertaalkunde, yol. $28, n^{\circ} 1$, p. 13.
Intent, vol. $28, \mathrm{n}^{\circ} 3$, p. 245

Inter-paragraph transition, vol. $28, n^{\circ} 3$, p. 268.

Inter-sentence transition, vol. $28, n^{\circ} 3$, p. 268

Interactive videodisc/conversation simulator, vol. $28, n^{\circ} 3$, p. 315 .

Interference, vol. 28, no $^{\circ}$, p. 270 .

Interior curve, vol. $28, \mathrm{n}^{\circ} 4$, p. 362

Intermediate technology, vol. $28, n^{\circ} 1$, p. 9.

International Association for Semiotics Studies, vol. $28, n^{\circ} 3$, p. 300 .

International Committee of Literary Authors, vol. $28, n^{\circ} 1$, p. 86

International Confederation of Authors' and Composers' Societies, vol. $28, n^{\circ} 1$, p. 86

International Federation of Translators, vol. 28 $\mathrm{n}^{\circ} 1$, p. 86.

International terminology, vol. $28, \mathrm{n}^{\circ} 2$, p. 222.

Interpretation in Japan, vol. $28, \mathrm{n}^{\circ} 1$, p. 95.

Interpreting, vol. $28, n^{\circ} 3$, p. 300 .

Interseasonal storage, vol. $28, \mathrm{n}^{\circ} 3$, p. 291

Intranslability of a hebrew text, vol. $28, n^{\circ} 1$, p. 57.

Inuit, vol. $28, \mathrm{n}^{\circ} 2$, p. 153 .

Inuktitut, vol. $28, \mathrm{n}^{\circ} 2$, p. 153.

Invent, vol. $28, n^{\circ} 3$, p. 254

Invitation to tender, vol. $28, \mathrm{n}^{\circ} 4$, p. 375 .

Irrelevant, vol. $28, \mathrm{n}^{\circ} 2$, p. 117

ISA 37 , vol. $28, \mathrm{n}^{\circ} 2$, p. 222.

ISO/TC 37, vol. $28, \mathrm{n}^{\circ} 2$, p. 222.

ISS, vol. $28, \mathrm{n}^{\circ} 1$, p. 109.

-ive, vol. $28, n^{\circ} 2$, p. 159

Japan Interpreter, The, vol. 28, $\mathrm{n}^{\circ} 1$, p. 107

Japan PEN, vol. 28, no 1, p. 107

Japan Society of Translators, vol. $28, \mathrm{n}^{\circ} 1$, p. 106.

Japanese history, vol. $28, n^{\circ} 1$, p. 97.

Japanese language, vol. $28, \mathrm{n}^{\circ} 1$, p. 95 .

Japanese publisher, vol. $28, n^{\circ} 1$, p. 104

Japanese sentences, vol. $28, n^{\circ} 1$, p. 109

Jeweller's piercing saw, vol. $28, n^{\circ} 4$, p. 365 .

Jingle, vol. $28, n^{\circ} 4, p, 405$.

JISTA, vol. $28, \mathrm{n}^{\circ} 1, \mathrm{p}, 7$

Joining saw, vol. 28, n $^{\circ} 4$, p. 360 .

Joining tools, vol. $28, n^{0} 4$, p. 359

Jointing, vol. 28, no 3, p. 293

Journal, vol. $28, \mathrm{n}^{\circ} 1$, p. 78 .

Journal of the ISTA, vol. $28, n^{\circ} 1$, p. 7

JST, vol. 28, no $^{\circ} 1$, p. 106

JST Bulletin, vol. $28, \mathrm{n}^{\circ} 1$, p. 107.

Juxtaglomerular apparatus, vol. $28, n^{\circ} 4$, p. 380 .

Kaiseijo, vol. $28, n^{\circ} 1$, p. 97

Kanii, vol. $28, n^{\circ} 1$, p. 95

Kanuri, vol. 28 , no 1 , p. 27

Kerf, vol, 28, no 2, p. 194

$\mathrm{KHz}$, vol. $28, \mathrm{n}^{\circ} 2$, p. 191.

Kilohertz, vol. $28, \mathbf{n}^{\circ} 2$, p. 191

Kit, vol. 28 , no 4, p. 404

Know variable, vol. 28 , no 4, p. 366

Korotkoff's sound, vol. $28, n^{\circ} 4$, p. 381 .

Kulliyet al-Alsun, vol. $28, n^{\circ} 1$, p. 10. 
Lake, vol. $28, \mathrm{n}^{\circ} 1$, p. 78.

Language, vol. $28, n^{\circ} 3$, p. 270 ; vol. $28, n^{\circ} 3$, p. 272.

Language Bureau, vol. $28, \mathrm{n}^{\circ} 2$, p. 153.

Language learning, vol. 28 , no 3 , p. 307 .

Language phenomena, vol. 28 , no 1, p. 68 .

Language Studies Unit, vol. 28 , no 1 , p. 13.

Language-transaction system, vol. $28, n^{\circ} 3$,

Latitude in translation, vol. $28, n^{\circ} 3$, p. 259.

LC, vol. $28, n^{\circ} 2$, p. 219.

Leading words, vol. $28, n^{\circ} 1$, p. 58

Leak, vol. $28, n^{\circ} 3$, p. 276.

Legal action, vol. $28, \mathrm{n}^{\circ} 2$, p. 173.

Legal proceeding, vol. 28 , n० 2 , p. 173.

Legislative Assembly Northwest Territories, vol. $28, \mathrm{n}^{\circ} 2$, p. 153.

Letter of agreement, vol. $28, \mathrm{n}^{\circ} 1$, p. 86.

Library of Congress, vol. $28, n^{\circ} 2$, p. 219

Light weight fret saw, vol. $28, n^{\circ} 4$, p. 365.

Linguistic entity, vol. $28, n^{\circ} 2$, p. 120 .

Linguistic-stylistic phenomena, vol. $28, \mathrm{n}^{\circ} 1$, p. 68 .

Link, vol. 28 , no 3 , p. 300.

Linked, vol. $28, \mathrm{n}^{\circ} 2$, p. 205

Liquid collector, vol. $28, n^{\circ} 3$, p. 289.

Liquid-expansion thermometer, vol. $28, n^{\circ} 3$ p. 298.

Liquid type collector, vol. 28, no 3, p. 289.

Liquor, vol. 28, no 2, p. 178.

Listening room, vol. $28, \mathrm{n}^{\circ} 2$, p. 192.

Literalness, vol. $28, n^{\circ} 3$, p. 254

Literary translation, vol. $28, n^{\circ} 3$, p. 300 .

Literary translation in Nigeria, vol. $28, n^{\circ} 1$, p. 27.

Literary translators, vol. $28, n^{\circ} 1$, p. 85 .

Locant, vol. $28, \mathrm{n}^{\circ} 2$, p. 167.

Locate, vol. $28, \mathrm{n}^{\circ} 3$, p. 254.

Log saw, vol. $28, n^{\circ} 4$, p. 363 .

Logging, vol. $28, n^{\circ} 4$, p. 362 .

Logic system, vol. $28, \mathrm{n}^{\mathrm{D}} 3$, p. 315.

Long term storage, vol. 28, no $^{\circ}$, p. 291.

Loucheux, vol. 28, n $^{\circ} 2$, p. 154.

Loud speaker, vol. 28 , no 2 , p. 191.

Loudness, vol. $28, n^{\circ} 4$, p. 401 .

Low audio frequency, vol. $28, n^{\circ} 2$, p. 191.

Lymphatic system, vol. 28, no 4, p. 379.

Machine aids for translators, vol. $28, n^{\circ} 4$, p. 344.

Machine assisted translation, vol. $28, \mathrm{n}^{\circ} 2$, p. 221.

Machine-readable form, vol. $28, n^{\circ} 4$, p. 344 ; vol. $28, \mathrm{n}^{\circ} 4$, p. 345 .

Machine translation, vol. $28, n^{\circ} 2$, p. 209 ; vol. 28 , $n^{\circ} 3$, p. 270 ; vol. 28 , no 4 , p. 347 .

Magana Hausa, vol. 28, $\mathrm{n}^{\circ} 1$, p. 27.

Magnetic tape, vol. $28, n^{\circ} 4$, p. 344.

Mailing, vol. $28, n^{\circ} 4$, p. 404 .

Mainichi Prize, vol. 28, n० 1, p. 107.

Malay, vol. $28, n^{\circ} 1$, p. 11

Malignant, vol. $28, n^{\circ} 4$, p. 385.

Mandarin Chinese, vol. $28, \mathrm{n}^{\circ} 1$, p. 11 .

Manipulated variable, vol. 28 , no 4 , p. 366

Marshal, vol. 28 , no 1, p. 78 .

Masonry wall, vol. 28 , no 3 , p. 293

Master, vol. $28, n^{\circ} 4$, p. 405

MAT, vol. $28, n^{\circ} 2$, p. 221.

Matter, vol. $28, n^{\circ} 3$, p. 245.
Maximally adequate translation, deviation from, vol. $28, n^{\circ} 2$, p. 129 .

Meaning, vol. $28, n^{\circ} 3$, p. 244 ; vol. $28, n^{\circ} 3$, p. 270 ; vol. $28, n^{\circ} 3$, p. 315 .

Measuring unit, vol. 28 , n० 4 , p. 367 .

Mechanical operator, vol. 28, no 3, p. 299.

Media, vol. 28, no 4, p. 402.

Medical domain, vol. $28, n^{\circ} 3$, p. 315 .

Medium frequency, vol. $28, n^{\circ} 2$, p. 192.

Merchandiser, vol. $28, \mathrm{n}^{\circ} 4$, p. 403.

Merchant, vol. 28, no 1, p. 96.

METAL, vol. 28 , no 4 , p. 349.

Metal bow, vol. $28, n^{\circ} 4$, p. 364 .

Metal bow frame, vol. 28 , no 4 , p. 364.

METEO, vol. $28, n^{\circ} 1$, p. 15.

Meteo system, vol. 28, no 2, p. 209.

Methyl alcohol, vol. $28, \mathrm{n}^{\circ} 2$, p. 168 .

M.F., vol. 28, n० $^{\circ}$, p. 192.

Mikado, vol. 28, n $^{\circ} 1$, p. 98 .

Mild, vol. $28, n^{\circ} 4$, p. 385 .

Millsaw, vol. 28, no 2, p. 199.

Miss, vol. $28, \mathrm{n}^{\circ} 1$, p. 78 .

Mistranslation, vol. $28, n^{\circ} 3$, p. 259

Mobile library, vol. $28, n^{\circ} 3$, p. 316

Model, vol. 28 , no 3 , p. 315 .

Model of curriculum, vol. $28, n^{\circ} 3$, p. 315.

Moderate, vol. $28, n^{\circ} 4$, p. 385 .

Modern Arab awakening, vol. 28, no 1, p. 77

Modern man, vol. 28 , no 1 , p. 60 .

Modern translation theory, vol. $28, \mathrm{n}^{\circ} 1$, p. 16.

Modulation, vol. $28, \mathrm{n}^{\circ} 3$, p. 253.

Monitor, vol. $28, n^{\circ} 4$, p. 403

Monosyllabic language, vol. $28, \mathrm{n}^{\circ} 1$, p. 95.

Month, vol. $28, \mathrm{n}^{\mathrm{O}} 1$, p. 78 .

Morphological analysis of German, vol. $28, n^{\circ} 2$, p. 211.

Mote, vol. $28, n^{\circ} 2$, p. 158

Movable insulation, vol. $28, n^{\circ} 3$, p. 290 .

Mr., vol. 28 , no 1 , p. 78.

Mrs., vol. 28, no $^{\circ}$, p. 78

MT, vol. $28, n^{\circ} 2$, p. 209 ; vol. $28, n^{\circ} 4$, p. 347

MT system, vol. $28, n^{\circ} 1$, p. 15.

Mullion, vol. 28 , no 3 , p. 293.

Multimedial communication, vol. 28 , no 3 , p. 301.

Multiplicity of meaning, vol. $28, n^{\circ} 3$, p. 300 .

Multiplying affix, vol. $28, \mathrm{n}^{\circ} 2$, p. 170

Murmur, vol. $28, n^{\circ} 4$, p. 381 .

Music power output, vol. 28 , n० 2, p. 192.

Musical quality, vol. $28, n^{\circ} 2$, p. 192

Muting, vol. 28, $\mathrm{n}^{\circ} 4$, p. 405 .

Name of literary characters, vol. $28, n^{\circ} 1$, p. 78

Name of publications, vol. $28, \mathrm{n}^{\circ} 1, \mathrm{p} .78$.

Name of the initial, vol. $28, \mathrm{n}^{\mathrm{o}} 1$, p. 82 .

Naphthaleneacetic acid, vol. $28, \mathrm{n}^{\circ} 2$, p. 169

Narrow bladed, vol. $28, n^{\circ} 2$, p. 197.

Narrow bladed framed pit saw, vol. $28, n^{\circ} 4$, p. 358 .

NATI, vol. $28, n^{\circ} 1$, p. 31 .

National Cultural Identity, vol. 28, n० 1, p. 29.

National Institute of Foreign Studies, vol. $28, n^{\circ} 1$, p. 97.

National Language Centre, vol. $28, n^{\circ} 1$, p. 32. 
National Workshop on Automatic Translation, vol. $28, \mathbf{n}^{\circ} 1$, p. 15 .

Native authority, vol. $28, \mathrm{n}^{\circ} 1$, p. 23.

Natural frequency, vol. $28, \mathrm{n}^{\circ} 2$, p. 191.

Near miss, vol. 28 , no 2, p. 205.

Need for a interpreter, vol. $28, n^{\circ} 3$, p. 315.

New technology, vol. $28, n^{\circ} 3$, p. 315 .

Newsletter, vol. $28, \mathrm{n}^{\circ} 1$, p. 87.

Newspaper, vol. $28, \mathrm{n}^{\circ} 1$, p. 78

Nigeria, vol. $28, \mathrm{n}^{\circ} 1$, p. 27

Nigerian Association of Translators and Interpreters, vol. $28, n^{\circ} 1$, p. 14 ; vol. $28, n^{\circ} 1$, p. 31.

Nitric oxide, vol, 28, no 3 , p. 298.

Nitrogen dioxide, vol. $28, \mathrm{n}^{\mathrm{O}} 3$, p. 299.

Nitrogen peroxide, vol. $28, \mathrm{n}^{\circ} 3$, p. 299.

No-random grouping, vol. $28, \mathrm{n}^{\circ} 1, \mathrm{p} .68$.

No-translation, vol. $28, \mathrm{n}^{\circ} 3$, p. 262.

Node, vol. $28, \mathrm{n}^{\circ} 3$, p. 300 .

Nominal phrase analyser, vol. $28, n^{\circ} 2$, p. 212.

Non-error based teaching, vol. $28, \mathrm{n}^{\circ} 3$, p. 307.

Non-fictitious text, vol. $28, n^{\circ} 3$, p. 300 .

Non-verbal sign, vol. $28, n^{\circ} 3$, p. 300 .

Nor-, vol. $28, n^{\circ} 2$, p. 169 .

Normative semiotic theory of translation, vol. 28 , $n^{\circ} 3$, p. 300.

Norpiname, vol. $28, n^{\circ} 2$, p. 169

North, vol. $28, \mathrm{n}^{\circ} 2$, p. 128.

Northwest Territories, vol. $28, \mathrm{n}^{\circ} 2$, p. 153.

Notional grammar, vol. $28, \mathrm{n}^{\circ} 3$, p. 307.

NWT, vol. $28, n^{\circ} 2$, p. 153.

Object position, vol. $28, n^{\circ} 3$, p. 308.

Obscurity, vol. $28, \mathrm{n}^{\circ} 3$, p. 261 .

Ocean, vol. $28, n^{\circ} 1$, p. 78.

Off-specification deviation, vol. $28, n^{\circ} 4$, p. 367

Official tour, on, vol. $28, \mathrm{n}^{\circ} 1$, p. 23.

Oil, vol. 28, n $^{\circ} 2$, p. 153.

-olactone, vol. $28, n^{\circ} 2$, p. 169

-olide, vol. 28 , no 2 , p. 169.

Omission by summarizing, vol. $28, \mathrm{n}^{\circ} 3$, p. 261 .

Omission of excess verbiage, vol. $28, n^{\circ} 3$, p. 261

On-line translation system, vol. $28, n^{\circ} 2$, p. 212.

Operating temperature, vol. $28, \mathrm{n}^{\circ} 3$, p. 291.

Operator, vol. $28, \mathrm{n}^{\circ} 3$, p. 299.

Optic fundus, vol. $28, \mathrm{n}^{\circ} 4$, p. 387.

Optical character reader, vol. $28, n^{\circ} 4$, p. 344.

Optimal equivalence, vol. $28, \mathrm{n}^{\circ} 2$, p. 117.

Optimal translating, vol. $28, \mathrm{n}^{\circ} 2$, p. 117.

-or, vol. $28, \mathrm{n}^{\circ} 2$, p. 159.

Order of certiorari, vol. $28, n^{\circ} 4$, p. 371 .

Ordinary message, vol. $28, \mathrm{n}^{\circ} 3$, p. 301 .

Original sound, vol. $28, n^{\circ} 2$, p. 192.

Original writing, vol. $28, n^{\circ} 3$, p. 260.

Orsat analysis, vol. $28, n^{\circ} 3$, p. 298.

-ory, vol. $28, n^{\circ} 2$, p. 159.

Other languages, vol. $28, n^{\circ} 3$, p. 270.

Output, vol. 28 , no 4 , p. 366 .

Output quantity, vol. $28, \mathrm{n}^{\circ} 4$, p. 366 .

Output variable quantity, vol. $28, n^{\circ} 4$, p. 366 .

Overlap, vol. 28 , no 4 , p. 401 .
Pacific Ocean, vol. 28, no 1, p. 78.

Pack-shot, vol. 28, no 4, p. 403.

Package, vol. 28, no 4 , p. 399.

Parallel structure, vol. 28, no 3, p. 308.

Parent compound, vol. $28, n^{\circ} 2$, p. 167.

Passive solar system, vol. 28, no 3, p. 291.

Passive voice, vol. $28, n^{\circ} 3$, p. 307 ; vol. $28, n^{\circ} 3$, p. 308.

Patent translation, vol. $28, n^{\circ} 1$, p. 8 .

Pattern of meaning, vol. 28, no $^{\circ}$, p. 266.

Pay-TV, vol. 28, no 4, p. 405.

Peak power, vol. 28, no $^{\circ}$, p. 192.

Peak-to-peak power, vol. $28, n^{\circ} 2$, p. 192.

Pebble bed storage, vol. $28, n^{\circ} 3$, p. 291.

Pedagogic strategy, vol. $28, n^{\circ} 3$, p. 307.

Pedagogic syllabus, vol. $28, \mathrm{n}^{\circ} 3$, p. 307.

Pedagogical issue, vol. $28, \mathrm{n}^{\circ} 3, \mathrm{p} .315$.

Peg teeth, vol. 28, n 2 , p. 195 ; vol. $28, n^{\circ} 4$, p. 358.

Pegged teeth, vol. $28, n^{\circ} 4$, p. 358 .

Perdite, vol. $28, n^{\circ} 2$, p. 158.

Perdition, vol. $28, \mathrm{n}^{\circ} 2$, p. 159.

Peregrine falcon, vol. $28, \mathrm{n}^{\circ} 3$, p. 254.

Performance, vol. $28, n^{\circ} 3$, p. 294.

Personal name, vol. 28, $\mathrm{n}^{\circ} 1, \mathrm{p} .78$.

Personal name initials, vol. $28, \mathrm{n}^{\circ} 1$, p. 82.

Personal names with titles, vol. $28, n^{\circ} 1$, p. 78.

Personal names without titles, vol. $28, \mathrm{n}^{\circ} 1$, p. 78.

Perum Telekomunikasi, vol. 28, no 1, p. 9.

Phase-changing salt, vol. $28, n^{\circ} 3$, p. 290.

Phasing, vol. 28, no 2, p. 192 ; vol. $28, n^{\circ} 4$, p. 404.

Phenomena, vol. 28 , no 1 , p. 68.

Phenylmercury chloride, vol. $28, \mathrm{n}^{\circ} 2$, p. 168.

Phono cartridge, vol. 28, n० 2, p. 192.

Phonological-graphological problem, vol. $28, \mathrm{n}^{\circ} 1$, p. 73.

Photothermal conversion, vol. 28, no 3, p. 289.

Phrase translation, vol. $28, n^{\circ} 2$, p. 210 .

Physical quantity, vol. $28, n^{\circ} 4$, p. 369.

Pidgin English, vol. 28, no 1, p. 20.

Piercing saw, vol. 28 , n 2 , p. 197 ; vol. 28 , no 4 , p. 365 .

Piling up of lexations, vol. $28, n^{\circ} 1$, p. 49.

Pilipino, vol. $28, \mathrm{n}^{\circ} 1$, p. 11 .

Pilipino and Linguistics, vol. $28, \mathrm{n}^{\circ} 1, \mathrm{p} .13$.

Pit saw, vol. $28, n^{\circ} 2$, p. 198 ; vol. $28, n^{\circ} 4$, p. 358.

Pitch, vol. 28, no 2, p. 195.

Pivotal arm, vol. 28, no 2, p. 191.

Pivotal metathesized axis, vol. $28, \mathrm{n}^{\circ} 1$, p. 63.

Pivoting tonearm, vol. $28, \mathrm{n}^{\circ} 2$, p. 191 .

Placement of the speaker (enclosures), vol. 28, $n^{\circ} 2$, p. 191

Placement of the speaker systems, vol. 28, n० 2 , p. 191.

Plank saw, vol. $28, n^{\circ} 2$, p. 198.

Poetry, vol. 28, no 3 , p. 308 .

Polite suffixing, vol. $28, \mathrm{n}^{\circ} 1$, p. 101.

Post-testing, vol. 28, no 4, p. 403.

Potassium hydroxide solution, vol. $28, n^{\circ} 3$, p. 298.

Potential of the text, vol. $28, n^{\circ} 3$, p. 300 .

Power, vol. 28, no 2, p. 192.

Practicable, vol. $28, n^{\circ} 3$, p. 267.

Practical Experience of Machine Translation, vol. 28, no 2, p. 209.

Pragmatic, vol. $28, n^{\circ} 3$, p. 300 . 
Pre-drafting stage, vol. $28, n^{\circ} 3$, p. 265

Pre-testing, vol. $28, n^{0} 4$, p. 404

Pre-translation, vol. $28, n^{\circ} 4$, p. 348 .

Preamp, vol. $28, n^{\circ} 2$, p. 192.

Preamplifier, vol. 28, n० 2, p. 192.

Precipitation release, vol. $28, n^{\circ} 3$, p. 255.

Predicative-Componential Analysis, vol. 28, $\mathrm{n}^{\circ} 2$, p. 148.

Prefixing, vol. 28, no 1, p. 76.

Prescribed law, vol. 28 , no 4 , p. 366.

Pressure range, vol. $28, n^{\circ} 3$, p. 298.

Primed number, vol. $28, n^{\circ} 2$, p. 171

Principles of terminology, vol. $28, n^{\circ} 2$, p. 222.

Private bill, vol. 28, n$^{\circ} 4$, p. 371.

Private member's bill, vol. 28 , no 4 , p. 371 .

Problem encountered in interpretation, vol. 28 , $\mathrm{n}^{\circ} 3$, p. 316

Proceeding, vol. 28, n० 2, p. 172.

Process, vol. $28, n^{\circ} 4$, p. 365 .

Process control computer, vol. 28, n०4, p. 369.

Professional development, vol. $28, n^{\circ} 3$, p. 315 ; vol. $28, n^{\circ} 3$, p. 316 .

Professor, vol. 28, n $^{\circ} 1$, p. 78

Program in translation and interpretation, vol. 28 , no 3, p. 315

Prohibiting statute, vol. $28, n^{\circ} 4$, p. 370 .

Proper nouns, vol. 28 , no 1 , p. 71 .

Proposed standard, vol. $28, \mathrm{n}^{\circ} 3$, p. 272.

Province, vol. 28, n $^{\circ} 3$, p. 260

Public address, vol. 28, no $^{\circ}$, p. 405.

Public address-amplifier, vol. 28 , no 4 , p. 400.

Public bill, vol. $28, n^{0} 4$, p. 371 .

Publishers Association, vol. 28, no 1, p. 86.

Publisher's contracts, vol. 28 , no 1, p. 86 .

Publisher/translator contract, vol. $28, \mathrm{n}^{\circ} 1$, p. 86 .

PUDSIKLIKBANGTEL, vol. 28, n० 1, p. 9.

Pull saw, vol. 28, n०2, p. 196.

Pulmonary circulation, vol. $28, n^{\circ} 4$, p. 384.

Pulse wave, vol. $28, \mathrm{n}^{\circ} 4$, p. 377.

Purpose, vol. 28, n $^{\circ} 3$, p. 270.

Push saw, vol. 28, n $^{\circ} 2$, p. 196.

Quantization, vol. 28, no 4 , p. 369.

Quash, vol. 28 , no 4 , p. 375 .

Question mark, vol. 28, no 3, p. 259.

Quick-and-dirty information, vol. $28, \mathrm{n}^{\circ} 4$, p. 348

Radial tonearm, vol. $28, \mathrm{n}^{\circ} 2$, p. 191

Radicofunctional nomenclature, vol. $28, \mathrm{n}^{\circ} 2$, p. 168.

Radiorecorder, vol. 28 , na 4 , p. 404.

Rain penetration, vol. $28, n^{\circ} 3$, p. 294

Rake angle, vol. $28, n^{\circ} 2$, p. 195.

Raker teeth, vol. $28, \mathrm{n}^{\circ} 2$, p. 195.

Rate, vol. 28 , no 4, p. 369.

Rate of translatability, vol. $28, n^{\circ} 2$, p. 124.

Ratio, vol. $28, n^{\circ} 2$, p. 192.

Re-arrangement of ideas, vol. $28, n^{\circ} 3$, p. 263.

Readability indice, vol. 28 , n 3 , p. 316

Reader, effect on the, vol. 28 , no 3, p. 245

Reader-oriented, vol. $28, n^{\circ} 3$, p. 300.

Rearrangement of points, vol. 28, no 3, p. 263.
Received pronounciation, vol. 28 , no 1, p. 82 .

Receiver, vol. 28 , no 4 , p. 400

Receiver/transmitter, vol. 28 , no 3 , p. 249

Recomposition of a target language text, vol. 28 , $n^{\circ} 2$, p. 117.

Recomposition of target message, vol. $28, \mathrm{n}^{\circ} 3$, p. 301.

Reconciliation proceeding, vol. $28, \mathrm{n}^{\circ} 2$, p. 174.

Recording, vol. $28, n^{\circ} 2$, p. 191

Refitting, vol. 28, no 3, p. 290

Refrain, vol. $28, n^{\circ} 2$, p. 205.

Regional Language Centre, vol. 28, no 1, p. 10.

Relation, vol. 28 , no 3 , p. 300 .

RELC, vol. 28 , no 1 , p. 10

Release print, vol. 28 , n० 4 , p. 401

Relevance, vol. $28, n^{\circ} 2$, p. 117.

Relevance of the shared features, vol. $28, n^{\circ} 2$, p. 117.

Relevant, vol. 28 , no 2 , p. 117.

Relevant features, vol. 28 , no 2 , p. 116

Renin-angiotensin system, vol. 28 , no 4 , p. 379 .

Repeal, vol. 28 , no 4 , p. 375

Repeater, vol. $28, n^{\circ} 2$, p. 205.

Repetition, vol. $28, \mathrm{n}^{\circ} 3$, p. 308.

Replacement nomenclature, vol. 28, no 2, p. 169.

Replacement of the decomposed source text, vol. $28, \mathrm{n}^{\circ} 2$, p. 117

Report-from notes, vol. $28, \mathrm{n}^{\circ} 3$, p. 263.

Report-from-text, vol. 28 , n० 3 , p. 263.

Reported discourse, vol. 28, no $^{\circ} 3$, p. 249.

Reported speech, vol. 28 , no 3 , p. 249.

Rescind, vol. $28, n^{\circ} 4$, p. 375 .

Resonance frequency, vol. $28, n^{\circ} 2$, p. 191

Resonant frequency, vol. $28, n^{\circ} 2$, p. 191.

Restructuring, vol. $28, n^{\circ} 3$, p. 253.

Retribute, vol. $28, n^{\circ} 2$, p. 159.

Retribution, vol. $28, \mathrm{n}^{\circ} 2$, p. 159

Retrofitting, vol. 28 , no 3 , p. 290

Revision stage, vol. 28 , no 3 , p. 267.

Rhymer, vol. $28, n^{\circ} 2$, p. 205.

Rhyming doublet, vol. 28 , no 2 , p. 204

Rhyming doublets, vol. $28, n^{\circ} 2$, p. 204.

Right aortic arch, vol. $28, n^{\circ} 4$, p. 384.

Ripsaw, vol. 28 , no 4 , p. 359 ; vol. 28 , no 4 , p. 360.

Rise time, vol. 28 , no 2 , p. 192.

Rising time, vol. $28, \mathrm{n}^{\circ} 2$, p. 192.

River, vol. 28 , no 1, p. 78

Rock bed storage, vol. $28, n^{\circ} 3$, p. 291.

Rock bin, vol. 28 , no 3 , p. 289.

Rock storage bin, vol. $28, n^{\circ} 3$, p. 289.

Role of the interpreter in the bilingual courtroom, vol. 28 , no 3 , p. 315

Role of the translator, vol. $28, n^{\circ} 3$, p. 245.

Roof pond, vol. 28 , no 3 , p. 291.

Roof-rock, vol. 28, no 3, p. 299.

Root mean square, vol. $28, \mathrm{n}^{\circ} 2$, p. 192.

Rough, vol. $28, n^{\circ} 4$, p. 401.

Rough lay-out, vol. $28, n^{\circ} 4$, p. 401.

RP, vol. $28, \mathrm{n}^{\circ} 1$, p. 82 .

Rule for translation, vol. 28 , n० 3, p. 275.

Rule-of-thumb, vol. 28 , no 3 , p. 275.

Russian frame saw, vol. $28, n^{\circ} 2$, p. 198.

Russian pit saw, vol. $28, n^{\circ} 2$, p. 198. 
Salaried translator, vol. $28, n^{\circ} 1$, p. 88

Sampled value, vol. $28, n^{\circ} 4$, p. 369 .

Sampling process, vol. $28, n^{\circ} 4$, p. 369 .

Samurai, vol. $28, \mathrm{n}^{\circ} 1$, p. 96 .

-san, vol. $28, \mathrm{n}^{\circ} 1$, p. 96.

Satow, Ernest, vol. 28 , no 1 , p. 98

Saw, vol. $28, n^{\circ} 2$, p. 194 ; vol. $28, n^{\circ} 2$, p. 195 ; vol. $28, n^{\circ} 2$, p. 196 ; vol. $28, n^{\circ} 2$, p. 197 vol. $28, n^{\circ} 2$, p. 198

Saw dust, vol. $28, \mathrm{n}^{\circ} 2$, p. 194.

Saw kerf, vol. $28, n^{\circ} 2$, p. 194.

Saw up, vol. $28, n^{\circ} 4$, p. 359.

Scanner, vol. $28, n^{\circ} 4$, p. 404

Schlegel-Tieck Prize, vol. $28, n^{\circ} 1$, p. 86

School of Letters, vol. $28, \mathrm{n}^{\circ} 1$, p. 13.

Science of Translation, The, yol. $28, \mathrm{n}^{\circ} 1, \mathrm{p} .12$

Scientific writing, vol. $28, \mathrm{n}^{\circ} 3$, p. 261 ; vol. 28 , $n^{\circ} 3$, p. 275 .

Scitech translators, vol. 28 , no 1, p. 88

Scotland, vol. 28 , no 1 , p. 78 .

Scott-Mocrieff Prize, vol. 28, $\mathrm{n}^{\circ} 1$, p. 86.

Scribing saw, vol. $28, n^{\circ} 4$, p. 364.

Script, vol. $28, n^{\circ} 4$, p. 404.

Scroll saw, vol. $28, n^{\circ} 4$, p. 363 .

Scrollwork, vol. $28, n^{\circ} 4$, p. 364.

Sealing rock, vol. $28, n^{\circ} 3$, p. 299.

SEAMEO, vol. $28, \mathrm{n}^{\circ} 1$, p. 10.

Second heart sound, vol. $28, n^{\circ} 4$, p. 380.

Second-language learning, vol. $28, \mathrm{n}^{\circ} 3$, p. 273.

Second language teaching, vol. $28, n^{\circ} 3$, p. 307.

Selective radiation coating, vol. $28, \mathrm{n}^{\circ} 3$, p. 291

Selective surface, vol. $28, \mathrm{n}^{\circ} 3$, p. 291.

Self-draining system, vol. $28, \mathrm{n}^{\mathrm{o}} 3$, p. 291

Self improvement, vol. $28, \mathrm{n}^{\circ} 3$, p. 244.

Semantic, vol. $28, n^{\circ} 3$, p. 300 ; vol. $28, n^{\circ} 3$, p. 315.

Semantic-connotational range, vol. $28, n^{\circ} 1$,

Semi-systematic name, vol. $28, n^{\circ} 2$, p. 166.

Semi-trivial name, vol. $28, n^{\circ} 2$, p. 166.

Semilunar cusp, vol. $28, n^{0} 4$, p. 383

Semilunar valve, vol. $28, n^{0} 4$, p. 382.

Semiotic theory of translation, vol. $28, n^{\circ} 3$, p. 300 .

Semiotic/translation study, vol. $28, n^{\circ} 3$, p. 301

Semiotik und übersetzen, vol. $28, n^{\circ} 3$, p. 300

Semiotik und übersetzungskritik an übersetzungen belletristischer Texte, vol. $28, n^{\circ} 3$, p. 300 .

Semiotische Aspekte der übersetzung von ComicsTexten, vol. $28, n^{\circ} 3$, p. 301

Semitic language, vol. $28, n^{\circ} 1$, p. 58.

-sense, vol. $28, n^{\circ} 1$, p. 96.

Sense of audience, vol. $28, n^{\circ} 3$, p. 265.

Sensor, vol. $28, \mathrm{n}^{\circ} 4$, p. 367.

Sentence, vol. $28, n^{\circ} 3$, p. 268 .

Sentence understanding, vol. $28, \mathrm{n}^{\circ} 2$, p. 210

Set, vol. 28, no 2, p. 194.

Set point, vol. $28, n^{\circ} 4$, p. 366

Set point signal, vol. $28, n^{\circ} 4$, p. 369.

Set-point station, vol. $28, n^{\circ} 4$, p. 369

Set, to, vol. $28, n^{\circ} 2$, p. 194.

Set value, vol. $28, n^{\circ} 4$, p. 366

Setting, vol. $28, \pi^{\circ} 2$, p. 194

Severe, vol. $28, n^{\circ} 4$, p. 385.

Shakespeare Translation, vol. $28, \mathfrak{n}^{\circ} 1$, p. 100

Shepherd's dog, vol. $28, n^{\circ} 3$, p. 309.

Shift, vol. $28, n^{\circ} 2$, p. 129.
Shift of relevancy, vol. $28, \mathrm{n}^{\circ} 2, \mathrm{p} .122$.

Short term storage, vol. $28, n^{\circ} 3$, p. 291.

Shunt, vol. $28, n^{\circ} 4$, p. 402 .

Side chain, vol. $28, n^{\circ} 2$, p. 170.

Siemens Language Services, vol. 28 , n ${ }^{\circ} 4$, p. 349

Sight translation, vol, $28, \mathrm{n}^{\circ} 1$, p. 101 ; vol. 28 , $n^{\circ} 3$, p. 316.

Sign languages, vol. 28, no $^{\circ} 3$, p. 315 .

Signal-to-noise ratio, vol. $28, n^{\circ} 2$, p. 192.

Simple lay-out, vol. $28, n^{\circ} 4$, p. 401 .

Simulinternational, vol. $28, \mathrm{n}^{\circ} 1$, p. 109

Simultaneous interpretation, vol. $28, n^{\circ} 3$, p. 315

Simultaneous interpreting, vol. $28, \mathrm{n}^{\circ} 1$, p. 109 ; vol. $28, n^{\circ} 4$, p. 409 .

Singapore Translators and Interpreters Union, vol. $28, n^{\circ} 1$, p. 14.

Single inverted commas, vol. $28, \mathrm{n}^{\circ} 1, \mathrm{p} .76$.

Single meaning, vol. $28, \mathrm{n}^{\circ} 3$, p. 300 .

Single standard, vol. 28, n $^{\circ} 3$, p. 272 .

SITU, vol. $28, \mathrm{n}^{\circ} 1$, p. 14.

Siuraujaaqtuq, vol. 28, no $^{\circ}$, p. 153

Skew back saw, vol. $28, n^{\circ} 2$, p. 194.

Skew teeth, vol. $28, n^{\circ} 2$, p. 195 ; vol. $28, n^{\circ} 4$, p. 359.

Sliding frame, vol. $28, n^{\circ} 4$, p. 365 .

Smith's frame saw, vol. $28, n^{\circ} 4$, p. 365 .

S/N ratio, vol. $28, n^{\circ} 2$, p. 192 .

Social act, vol. $28, \mathrm{n}^{\circ} 3$, p. 245.

Social relation, vol. $28, n^{\circ} 3$, p. 245 .

Society of Authors, vol. 28, no 1, p. 85.

Solar architecture, vol. $28, \mathrm{n}^{\circ} 3$, p. 289.

Solar collector, vol. 28, no $^{\circ}$, p. 289.

Solar engineering, vol. $28, n^{\circ} 3$, p. 290.

Solar gain, vol. $28, n^{\circ} 3$, p. 289.

Solar heat storage, vol. $28, n^{\circ} 3$, p. 289

Solar heating, vol. 28, no $^{\circ}$, p. 289.

Solar receiver, vol. $28, \mathrm{n}^{\circ} 3$, p. 289.

Solar technology, vol. 28, no 3, p. 290.

Solar wall, vol. $28, n^{\circ} 3$, p. 290.

Some notes on machine aids for translators, vol. $28, n^{\circ} 4$, p. 344 .

Sony, vol, $28, \mathrm{n}^{\circ} 1$, p. 109

Sound, vol. $28, n^{\circ} 2$, p. 192 ; vol. $28, n^{\circ} 4$, p. 380 .

Sound source, vol. $28, n^{\circ} 2$, p. 192.

Sound system, vol. $28, n^{\circ} 2$, p. 186 ; vol. $28, n^{\circ} 2$, p. 191.

Sound wave, vol. $28, n^{\circ} 2$, p. 192

Source-language expression, vol. $28, n^{\circ} 3$, p. 253.

Source text, vol. 28 , no 4 , p. 344

South Pacific Bureau for Economic Cooperation, vol. $28, n^{\circ} 1$, p. 19.

Southeast Asian Ministers of Education Organization, vol. 28, $\mathrm{n}^{\circ} 1$, p. 10.

Soviet scientific and technical literature, vol. 28 , $n^{\circ} 1$, p. 6

Spalling, vol. $28, n^{\circ} 3$, p. 294.

Spandrel, vol. $28, \mathrm{n}^{\circ} 3$, p. 294

Speaker, vol. $28, n^{\circ} 2$, p. 191.

Speaker enclosure, vol. $28, n^{\circ} 2$, p. 191

Speaker system, vol. $28, \mathrm{n}^{\circ} 2$, p. 191.

SPEC, vol. $28, \mathrm{n}^{\circ} 1$, p. 19

Specialized, translators, vol. $28, n^{\circ} 1$, p. 87 .

Specific tool, vol. $28, n^{\circ} 4$, p. 344.

Specific volume, vol. $28, \mathrm{n}^{\circ} 3$, p. 299.

Spigot, vol. $28, n^{\circ} 4$, p. 360.

Spirits, vol. 28, no $^{\circ}$, p. 178. 
Split run, vol. $28, \mathrm{n}^{\circ} 4$, p. 406.

Split run test, vol. $28, n^{\circ} 4$, p. 406.

Sponsor, vol. $28, n^{\circ} 4$, p. 401.

Sponsoring, vol. $28, n^{\circ} 4$, p. 403

Spreading, vol. $28, n^{\circ} 3$, p. 267

Sputnik, vol. $28, n^{\circ} 1$, p. 6 .

Staff translators, vol. $28, \mathrm{n}^{\circ} 1$, p. 88.

Stagnation temperature, vol. $28, \mathrm{n}^{\circ} 3$, p. 291.

Standard, vol. $28, n^{\circ} 3$, p. 272

Standardization, vol. $28, \mathrm{n}^{\circ} 3$, p. 272.

State, vol. 28 , no 1 , p. 78 .

Steering variable, vol. $28, n^{\circ} 4$, p. 366.

Stereo effect, vol. $28, n^{\circ} 2$, p. 191.

Stereo(phony), vol. 28, no 2, p. 192.

Stock-shot, vol. 28, n $^{\circ} 4$, p. 402.

Storage bin, vol. $28, n^{\circ} 3$, p. 289.

Storage tank, vol. 28 , no 3 , p. 289.

Storage tray, vol. $28, n^{\circ} 3$, p. 290.

Story-board, vol. $28, n^{\circ} 4$, p. 404.

Straight back saw, vol. $28, n^{\circ} 2$, p. 194

Straight line tonearm, vol. $28, \mathrm{n}^{\circ} 2$, p. 191

Stretcher, vol. $28, \mathrm{n}^{\circ} 2$, p. 196.

Styling element, vol. $28, n^{\circ} 4$, p. 405 .

Stylist, vol. $28, n^{\circ} 4$, p. 405.

Subject classification, vol. $28, n^{\circ} 2$, p. 222.

Subject position, vol. $28, n^{\circ} 3$, p. 308

Sublanguage, vol. $28, \mathrm{n}^{\circ} 2$, p. 210

Subsequent reference, vol. $28, n^{\circ} 3$, p. 308

Substituent, vol. $28, \mathrm{n}^{\circ} 2$, p. 167.

Substitutive nomenclature, vol. $28, \mathrm{n}^{\circ} 2$, p. 167.

Substractive nomenclature, vol. $28, \mathrm{n}^{\circ} 2$, p. 168.

Sugar, vol. $28, n^{\circ} 2$, p. 153

Suit, vol. 28 , no 2 , p. 173 .

Summarizing, vol. $28, \mathrm{n}^{\circ} 3$, p. 263.

Summary, vol. $28, \mathrm{n}^{\circ} 3$, p. 263.

Supervisory control, vol. $28, n^{\circ} 4$, p. 367.

Supervisory digital control, vol. $28, \mathrm{n}^{\mathrm{o}} 4$, p. 367

Supplementary meaning, vol. $28, \mathrm{n}^{\circ} 3$, p. 300 .

Sweep saw, vol. $28, n^{\circ} 4$, p. 361.

Swivel blade, vol. 28, no $^{\circ}$, p. 360

Swivel knob, vol. $28, n^{\circ} 4$, p. 360

Syntactic, vol. $28, n^{\circ} 3$, p. 300

Syntactic structure, vol. $28, n^{\circ} 3$, p. 315.

System, vol. $28, n^{\circ} 2$, p. 209.

System of concepts and their representation, vol. $28, n^{\circ} 2$, p. 222 .

Systematic name, vol. $28, n^{\circ} 2$, p. 166

Systemic, vol. $28, n^{\circ} 4$, p. 385 .

Systemic circulation, vol. $28, n^{\circ} 4$, p. 384

TA, vol. 28 , no 1 , p. 86 .

Tagalog, vol. 28 , no 1 , p. 11

Tang, vol. $28, \mathrm{n}^{\circ} 4$, p. 362 .

Tanzanian Association of Translators, vol. 28, no 1 , p. 14.

Tape deck, vol. $28, \mathrm{n}^{\circ} 2$, p. 186 ; vol. $28, \mathrm{n}^{\circ} 2$, p. 192.

Target-language equivalent, vol. $28, n^{\circ} 3$, p. 253.

TAUM-A viation, vol. $28, n^{\circ} 2$, p. 210

Tax drive, vol. $28, \mathrm{n}^{\circ} 1$, p. 23.

Teaching for understanding, vol. $28, n^{\circ} 3$, p. 316

Teaching of english grammar, vol. $28, n^{\circ} 3$, p. 306

Teaching tool, vol. $28, n^{\circ} 3$, p. 307

Teaser, vol. $28, n^{\circ} 4$, p. 400 .
Teasing, vol. 28, n० 4, p. 400.

Tech-writer, vol. 28, n $^{\circ} 4$, p. 344.

Technical documentation, the world's, vol. 28 , $n^{\circ} 1$, p. 6.

Technical Translation Group, vol. $28, \mathrm{n}^{\circ} 1$, p. 88.

Technical translator, vol. $28, n^{\circ} 4$, p. 344.

Technology, vol. $28, n^{\circ} 1$, p. 9 .

Technology, diffusion of new, vol, $28, n^{\circ} 1$, p. 9.

Teeth, vol. $28, n^{\circ} 2$, p. 195.

Telecommunication technology, vol. $28, n^{\circ} 3$, p. 270.

Telecommunications Directorate of Indonesia, vol. $28, n^{\circ} 1$, p. 9.

Teleprompter, vol. $28, n^{\circ} 4$, p. 405 .

Temporal names, vol. $28, \mathrm{n}^{\circ} 1$, p. 78 .

Tensioning cord, vol. $28, \mathbf{n}^{\circ} 2$, p. 196

Terminology, vol. $28, n^{\circ} 2$, p. 222 ; vol. $28, n^{\circ} 4$ p. 345.

Terminology research, vol. $28, n^{\circ} 4$, p. 346.

Terminology work in Japan, vol. $28, \mathrm{n}^{\circ} 2$, p. 221.

Text, vol. $28, n^{\circ} 1$, p. 58

Text analysis, vol. 28, n $^{\circ} 3$, p. 300 .

Text constitution, vol. $28, \mathrm{n}^{\circ} 3$, p. 300

Text-creator, vol. $28, \mathrm{n}^{\circ} 3$, p. 272.

Text difficulty, vol. $28, n^{\circ} 3$, p. 316

Text editing, vol. $28, n^{\circ} 2$, p. 210 ; vol. $28, n^{\circ} 4$, p. 345 .

Text-fetishism, vol. $28, n^{\circ} 3$, p. 267

Text-prism, vol. $28, \mathrm{n}^{\circ} 1$, p. 57 .

Text-specific glossary, vol. 28 , no 4 , p. 346

Text-type, vol. $28, n^{\circ} 3$, p. 300 .

Text $\mathrm{x}$, vol. $28, \mathrm{n}^{\circ} 3, \mathrm{p} .244$

Text y, vol. $28, \mathrm{n}^{\circ} 3$, p. 244.

Textual Analysis, vol. 28, n $^{\circ} 1$, p. 13.

Textus, vol. $28, \mathrm{n}^{\circ} 1$, p. 68 .

TG, vol. 28 , no 1 , p. 87

TGNL, vol. $28, \mathrm{n}^{\circ} 1$, p. 87.

Thai, vol. $28, \mathrm{n}^{\mathrm{o}} 1$, p. 11

That is why, vol. $28, n^{\circ} 3$, p. 268

The Translators and Interpreters Educational Society, vol. $28, n^{\circ} 3$, p. 315 .

Theoretical air, vol. $28, n^{\circ} 3$, p. 298.

Theoretical air requirement, vol. $28, \mathrm{n}^{\circ} 3$, p. 298.

Theoretical course, vol. $28, n^{\circ} 3$, p. 308 .

Theoretical issue, vol. $28, n^{\circ} 3$, p. 315 .

Theory of language, vol. $28, n^{\circ} 3$, p. 308.

Theory of translation and semiotics, vol. $28, n^{\circ} 3$, p. 300.

Thermosiphoning solar collector, vol. $28, \mathrm{n}^{\circ} 3$, p. 290.

Threat, vol. $28, n^{\circ} 3$, p. 268.

T.I.E.S., vol. $28, n^{0} 3$, p. 315 .

T.I.E.S. Symposium, vol. $28, n^{\circ} 3$, p. 315.

Timbre, vol. 28, no $^{\circ}$, p. 192

Time, vol. $28, n^{\circ} 2$, p. 192 .

Time-code, vol. 28, n $^{\circ} 4$, p. 401.

Title, vol. $28, \mathrm{n}^{\circ} 1$, p. 78

To sponsor, vol. $28, n^{\circ} 4$, p. 401.

Toggle stick, vol. 28, no $^{\circ}$, p. 196

Tokyo Bay, vol. $28, n^{\circ} 1$, p. 97.

Tokyo Institute of Foreign Studies, vol. $28, n^{\circ} 1$, p. 108.

Tone color, vol. $28, n^{\circ} 2$, p. 192.

Tone language, vol. 28 , no 1, p. 95

Tongue officer, vol. $28, n^{\circ} 1$, p. 99.

Top end of cheek, vol. $28, \mathrm{n}^{\circ} 2$, p. 196. 
Topic/comment, vol. 28 , no 2, p. 207

Toward a Semiotic Theory of Literary Translating, vol. $28, n^{0} 3$, p. 300 .

Traditional proper noun, vol. $28, \mathrm{n}^{\circ} 1$, p. 77

Trainee, vol. 28 , no 3, p. 244 .

Trainer, vol. 28 , no 3 , p. 244.

Training, vol. $28, n^{\circ} 3$, p. 244 ; vol. 28 , n० 3 , p. 315 .

Training material, vol. 28 , no 3 , p. 316

Training of in-house personnel, vol. $28, n^{\circ} 3$, p. 316.

Trans-, vol. 28, n $^{\circ} 2$, p. 158 .

Transaction, vol. 28, n $^{\circ} 3$, p. 270

Transcoding, vol. $28, n^{\circ} 3$, p. 271 ; vol. $28, n^{\circ} 3$, p. 276.

Transcription turn-table, vol. $28, n^{\circ} 2$, p. 186 vol. $28, n^{\circ} 2$, p. 192

Transfer, vol. $28, n^{\circ} 3$, p. 301 .

Transfer of a message, vol. $28, n^{\circ} 3$, p. 246.

Transfer of technology, vol. $28, n^{\circ} 1$, p. 5

Transfer operation, vol. $28, n^{\circ} 3$, p. 301.

Transition, vol. $28, n^{\circ} 3$, p. 268

Translability, vol. 28 , n० 2 , p. 116 .

Translatability, vol. $28, \mathrm{n}^{\circ} 2$, p. 124.

Translating, vol. $28, n^{\circ} 2$, p. 116.

Translating dictionary, vol. $28, n^{\circ} 3$, p. 274

Translation, vol. $28, n^{\circ} 2$, p. 117 ; vol. $28, n^{\circ} 3$, p. 262 ; vol. $28, n^{\circ} 3$, p. 315 .

Translation and interpretation in Japan, vol. 28 , no 1 , p. 95 .

Translation and semiotics, vol. 28 , no 3, p. 300 .

Translation course, vol. $28, n^{\circ} 3$, p. 307.

Translation criticism, vol. $28, n^{\circ} 3$, p. 300

Translation critique, vol. $28, \mathrm{n}^{\circ} 1$, p. 13 .

Translation equivalence, vol. $28, \mathrm{n}^{\circ} 2, \mathrm{p} .124$.

Translation factory, vol. $28, \mathrm{n}^{\circ} 1$, p. 106.

Translation is an independant discipline, vol. 28 , $n^{\circ} 3$, p. 306 .

Translation like, vol. $28, n^{\circ} 3$, p. 264.

Translation-oriented Textual Analysis, vol. 28, $n^{\circ} 1$, p. 13 .

Translation process, vol. $28, n^{\circ} 3$, p. 301 .

Translation program, vol. $28, n^{\circ} 3$, p. 307.

Translation-relevant text, vol. $28, n^{\circ} 3$, p. 300 .

Translation system, vol. $28, n^{\circ} 2$, p. 212

Translation teaching, vol. $28, \mathrm{n}^{\circ} 1$, p. 5 .

Translation theory, vol. $28, \mathrm{n}^{\circ} 1$, p. 12 ; vol. 28 , $\mathrm{n}^{\circ} 1$, p. 16.

Translation und nonverbale Kommunikation, vol. 28 , no 3 , p. 300 .

Translation, United Kingdom, vol. 28 , n ${ }^{\circ} 1$, p. 85 .

Translational consideration, vol. $28, \mathrm{n}^{\circ} 2$, p. 116 .

Translator as rapporteur, the, vol. $28, n^{\circ} 3$, p. 244.

Translator-rapporteur, vol. $28, n^{\circ} 3$, p. 266.

Translator, role of the, vol. 28 , no 3, p. 245

Translators Association, vol. $28, \mathrm{n}^{\circ} 1$, p. 85.

Translators' Final Examination, vol. 28, no 1 p. 87.

Translator's footnote, vol. $28, n^{\circ} 3$, p. 259

Translators' Guild Ltd., vol. 28, no 1, p. 86.

Translators' Intermediate Examination, vol. 28, $n^{\circ} 1$, p. 87 .

Translators News, vol. $28, n^{\circ} 1$, p. 86

Translator's organizations, vol. $28, \mathrm{n}^{\circ} 1, \mathrm{p} .85$.

Transliteration, vol. 28, no $^{\circ}$, p. 70.
Transliteration of English proper nouns, vol. 28, no 1, p. 71 .

Transliteration of english proper nouns into Arabic, vol. 28, n $^{\circ} 1$, p. 70

Transliteration of proper nouns, vol. $28, n^{\circ} 1$, p. 71.

Transliteration versus translation, vol. $28, \mathrm{n}^{\circ} 1$, p. 78.

Transmote, vol. $28, n^{\circ} 2$, p. 158

Transposition, vol. $28, \mathrm{n}^{\circ} 3$, p. 253.

Triconsonantal root, vol. $28, \mathrm{n}^{\circ} 1$, p. 59.

Tricuspid valve, vol. $28, n^{\circ} 4$, p. 382 .

Trilingual project, vol. $28, n^{\circ} 3$, p. 315 .

Trimethylaluminum, vol. 28 , no 2 , p. 168 .

Trivial name, vol. $28, n^{\circ} 2$, p. 166

Trombe wall, vol. 28 , no 3 , p. 290

True doublet, vol. $28, n^{\circ} 2$, p. 205.

TTG, vol. 28 , no 1, p. 88 .

Tube-in-plate absorber, vol. 28 , no 3, p. 291.

Tube-in-sheet absorber, vol. 28, no 3, p. 291.

Tube-in-strip, vol. $28, \mathrm{n}^{\circ} 3$, p. 291.

Tubular bow saw, vol. 28 , no 2 , p. 197 ; vol. 28 , no 4 , p. 363

Tubular frame-saw, vol. $28, \mathrm{n}^{\circ} 4$, p. 363.

Tubular steel bow, vol. 28 , no 4 , p. 363 .

Tuner, vol. $28, n^{\circ} 4$, p. 405 .

Turning saw, vol. 28 , n० 4 , p. 361

Turntable, vol. $28, n^{\circ} 2$, p. 186 ; vol. $28, n^{\circ} 2$, p. 192.

Two-eared hearing, vol. 28 , no 2 , p. 191.

Two-eared listening, vol. 28 , n० 2 , p. 191

Two-man pit saw, vol. $28, \mathrm{n}^{\circ} 2$, p. 198

Two-man saw, vol. $28, n^{\circ} 4$, p. 358 .

Two-tier system, vol. 28 , no 1 , p. 108

UK universities, vol. 28 , no 1, p. 89.

Umbrella term, vol. $28, \mathrm{n}^{\circ} 1$, p. 64 .

Unable to reproduce, vol. 28 , no 3 , p. 258.

Undertranslation, vol. $28, n^{\circ} 3$, p. 259.

Unesco Recommendation, vol. $28, \mathrm{n}^{\circ} 1$, p. 88.

Unit heater, vol. 28 , no 3 , p. 289.

United Kingdom, vol. $28, \mathrm{n}^{\circ} 1$, p. 85 .

University of Kyoto, vol. $28, n^{\circ} 2$, p. 221.

University of Toronto Library Automation Systems, vol. 28 , no 2 , p. 219 .

Unoriginal writing, vol. $28, n^{\circ} 3$, p. 260 .

Untranslatableness of poetry, vol. $28, n^{\circ} 3$, p. 309.

Uqsualuk, vol. $28, \mathrm{n}^{\circ} 2$, p. 153.

Urban community, vol. $28, n^{\circ} 4$, p. 376.

Urban council, vol. $28, n^{\circ} 1$, p. 23.

UTLAS, vol. $28, n^{\circ} 2$, p. 219 .

Valvula similunaris, vol. $28, \mathrm{n}^{\circ} 4$, p. 383 .

Vapour barrier, vol. 28, no 3, p. 294.

Variable, vol. $28, n^{\circ} 4$, p. 366 .

Variable physical quantity, vol. $28, n^{\circ} 4$, p. 366.

Ventricular systole, vol. $28, n^{\circ} 4$, p. 377.

Verb phrase analyzer, vol. $28, n^{\circ} 2$, p. 212.

Verb system, vol. $28, n^{\circ} 3$, p. 307.

Verbal creativity, vol. $28, n^{\circ} 4$, p. 346.

Verbal sign, vol. $28, n^{\circ} 3$, p. 300 .

Verbiage, vol. $28, n^{\circ} 3$, p. 261. 
Vergleichende Textanalyse als Basis für die Entwicklung einer übersetzungsmethodik,

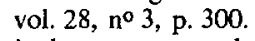

Vesicular murmur, vol. 28 , no 4 , p. 381 .

Viable, vol. $28, n^{\circ} 3$, p. 267.

Video-clip, vol. $28, n^{\circ} 4$, p. 400

Video display terminal, vol. $28, n^{\circ} 4$, p. 344.

Walkman, vol. $28, n^{\circ} 4$, p. 400 .

Warriors, vol. 28, n $^{\circ} 1$, p. 96.

Water heater used for central heating, vol. 28 , no 3, p. 299.

Water penetration, vol. $28, n^{\circ} 3$, p. 294

Water wall, vol. $28, n^{\circ} 3$, p. 290

Watts R.M.S., vol. $28, n^{\circ} 2$, p. 192.

Way of the Bushi, vol. 28, no 1, p. 96.

Web, vol. 28 , no 2 , p. 196.

Web saw, vol. 28, no 2, p. 196.

Week, vol. 28, n $^{\circ} 1$, p. 78 .

Wind driven rain, vol. $28, n^{\circ} 3$, p. 294.

Wine, vol. $28, n^{\circ} 2$, p. 178.

Withe, vol. 28, n $^{\circ} 3$, p. 294.

Wood saw, vol. $28, n^{\circ} 4$, p. 362 .

Woodcutter's saw, vol. $28, \mathrm{n}^{\circ} 4$, p. 362.

Word, vol. $28, n^{\circ} 3$, p. 245.

Word-by-word transcoding, vol. 28 , n॰ 3 , p. 276.

Word processing system, vol. $28, n^{\circ} 4$, p. 344 .

Word systems, vol. $28, \mathrm{n}^{\circ} 1$, p. 58 .

World's technical documentation, the, vol. 28 , no 1, p. 6.

Writing process, vol. $28, n^{\circ} 3$, p. 316 .

Writing skill, vol. $28, n^{\circ} 3$, p. 307 .

-yne, vol. 28, no 2, p. 169.

Yoruba, vol. 28, no $^{\circ} 1$, p. 27.

Zeichen, vol. 28 , no 3 , p. 300.

Zeichen oder Anzeichen? Probleme der AsTextanalyse im Blick auf die Übersetzung, vol. 28 , no 3 , p. 300 . 